\title{
Seltene Erkrankungen des vestibulären Labyrinths: von Zebras, Chamäleons und Wölfen im Schafspelz
}

\section{Rare Disorders of the Vestibular Labyrinth: of Zebras, Chameleons and Wolves in Sheep's Clothing}

\section{다 (i) $($ ) $€$}

Autor

Julia Dlugaiczyk

\section{Institut}

Klinik für Ohren-, Nasen-, Hals- und Gesichtschirurgie \& Interdisziplinäres Zentrum für Schwindel und neurologische Sehstörungen, Universitätsspital Zürich (USZ), Universität Zürich (UZH), Zürich, Schweiz

\section{Schlüsselwörter \\ Autoimmunerkrankungen des Innenohres, bilaterale Vestibulopathie, erweiterter Aqueductus vestibuli, intralaby- rinthäres Schwannom, Syndrome des «dritten Fensters», Tumoren des endolymphatischen Sacks}

\section{Key words}

autoimmune inner ear disease, bilateral vestibulopathy, endolymphatic sac tumor, enlarged vestibular aqueduct, intralabyrinthine schwannoma, "third window" syndromes

\section{Bibliografie}

Laryngo-Rhino-Otol 2021; 100: 1-40

DOI 10.1055/a-1349-7475

ISSN $0935-8943$

(c) 2021. The Author(s).

This is an open access article published by Thieme under the terms of the Creative Commons Attribution-NonDerivative-NonCommercial-License, permitting copying and reproduction so long as the original work is given appropriate credit. Contents may not be used for commecial purposes, or adapted, remixed, transformed or built upon. (https://creativecommons. org/licenses/by-nc-nd/4.0/)

(c) Georg Thieme Verlag KG, Rüdigerstraße 14, 70469

Stuttgart, Germany

Korrespondenzadresse

Prof. Dr. med. Julia Dlugaiczyk

Universitätsspital (USZ), Klinik für HNO- \& Gesichtschirurgie

Frauenklinikstraße 24

CH-8091 Zürich

Tel.: + 4144 / 2555550

julia.dlugaiczyk@usz.ch

\section{ZUSAMMENFASSUNG}

Die Differenzialdiagnose von Erkrankungen des vestibulären Labyrinths stellt eine grosse Herausforderung dar, da sich hinter dem sehr häufigen Leitsymptom «Schwindel» viele verschiedene und v. a. seltene Erkrankungen verbergen können. Der vorliegende Beitrag gibt einen Überblick über die für den HNOArzt wichtigen seltenen Erkrankungen des vestibulären Labyrinths ausgehend von ihrer klinischen Präsentation als akutes (AVS), episodisches (EVS) oder chronisches vestibuläres Syndrom (CVS). Der Schwerpunkt liegt dabei auf den EVS, sortiert nach ihrer Dauer und dem Vorhandensein von Triggern (Sekunden, ohne Trigger: Vestibularisparoxysmie, Tumarkin-Krise; Sekunden, lärm- und druckinduziert: Syndrome des «dritten Fensters»; Sekunden bis Minuten, positionsabhängig: seltene Varianten und Differenzialdiagnosen des benignen paroxysmalen Lagerungsschwindels; Stunden bis Tage, spontan: intralabyrinthäre Schwannome, Tumoren des endolymphatischen Sacks, Autoimmunerkrankungen des Innenohres). Des Weiteren werden seltene Differenzialdiagnosen eines AVS (Neuritis vestibularis inf., Otolithenfunktionsstörungen, vaskuläre Ursachen, akute bilaterale Vestibulopathie) und eines CVS (bilaterale Vestibulopathie) erläutert. Dabei werden insbesondere die entscheidenden diagnostischen Massnahmen für die Identifikation der einzelnen Krankheitsbilder und die Warnzeichen für potentiell gefährliche Ursachen (z. B. Labyrinthinfarkt/-blutung) dargelegt. Somit dient dieser Beitrag dem HNO-Arzt in Klinik und Praxis als eine Art «Vademecum» für die zügige Identifikation und zeitnahe Therapie seltener Erkrankungen des Gleichgewichtsorgans.

\section{ABSTRACT}

The differential diagnosis of vertigo syndromes is a challenging issue, as many - and in particular - rare disorders of the vestibular labyrinth can hide behind the very common symptoms of "vertigo" and "dizziness". The following article presents an overview of those rare disorders of the balance organ that are of special interest for the otorhinolaryngologist dealing with vertigo disorders. For a better orientation, these disorders are categorized as acute (AVS), episodic (EVS) and chronic vesti- 
bular syndromes (CVS) according to their clinical presentation. The main focus lies on EVS sorted by their duration and the presence/absence of triggering factors (seconds, no triggers: vestibular paroxysmia, Tumarkin attacks; seconds, sound and pressure induced: "third window" syndromes; seconds to minutes, positional: rare variants and differential diagnoses of benign paroxysmal positional vertigo; hours to days, spontaneous: intralabyrinthine schwannomas, endolymphatic sac tumors, autoimmune disorders of the inner ear). Furthermore, rare causes of AVS (inferior vestibular neuritis, otolith organ specific dysfunction, vascular labyrinthine disorders, acute bilateral vestibulopathy) and CVS (chronic bilateral vestibulopathy) are covered. In each case, special emphasis is laid on the decisive diagnostic test for the identification of the rare disease and "red flags" for potentially dangerous disorders (e. g. labyrinthine infarction/hemorrhage). Thus, this chapter may serve as a clinical companion for the otorhinolaryngologist aiding in the efficient diagnosis and treatment of rare disorders of the vestibular labyrinth.

\section{Inhaltsverzeichnis}

$1 . \quad$ Einleitung

1.1 Seltene Erkrankungen des vestibulären Labyrinths

1.1.1 Probleme

1.1.2 Lösungsansätze

1.2 "Gebrauchsanweisung» für diesen Beitrag

2. Akute vestibuläre Syndrome

2.1 Akute einseitige peripher-vestibuläre Unterfunktion

2.1.1 Neuritis vestibularis inferior

2.1.2 Akute einseitige Unterfunktion der Otolithenorgane

2.1.3 Therapie

2.2 Akute einseitige audiovestibuläre Funktionsstörung

2.2.1 Labyrinthinfarkt

2.2.2 Labyrinthblutung

2.2.3 Labyrinthitis

2.3 Akute beidseitige peripher-vestibuläre Funktionsstörung

3. Episodische vestibuläre Syndrome

3.1 Spontaner episodischer Schwindel (Sekunden)

3.1.1 Vestibularisparoxysmie

3.1.2 Tumarkin-Krisen

3.2 Lärm- und druckinduzierter episodischer Schwindel (Sekunden) - Syndrome des dritten Fensters

3.2.1 Pathophysiologie und Leitsymptome

3.2.2 Dehiszenz des superioren Bogenganges

3.2.3 "Near dehiscence"-Syndrom des superioren Bogenganges

3.2.4 Dehsizenz des posterioren Bogenganges

3.2.5 Dehiszenz des horizontalen Bogenganges

3.2.6 Erweiterter Aqueductus vestibuli/Saccus endolymphaticus

3.2.7 X-chromosomale familiäre Schwerhörigkeit mit Stapes-Gusher (DFNX2)

3.2.8 Differenzialdiagnosen von Syndromen des dritten Fensters

3.3 Positionsabhängiger Schwindel für Sekunden bis Minuten

3.3.1 Seltene Varianten des benignen paroxysmalen Lagerungsschwindels (BPLS)
3.3.2 Seltene Differenzialdiagnosen eines BPLS 20

3.4 Spontaner episodischer Schwindel (Stunden bis Tage) 20

3.4.1 Intralabyrinthäres Schwannom 20

3.4.2 Tumoren des endolymphatischen Sacks 22

3.4.3 Autoimmunerkrankungen des Innenohres 22

4 Chronische vestibuläre Syndrome 25

4.1 Bilaterale Vestibulopathie 25

4.1.1 Symptomatik 25

4.1.2 Klassifikation und Diagnostik 26

4.1.3 Ätiologie 26

4.1.4 Zusatzdiagnostik zur Ursachenabklärung 30

4.1.5 Therapie 30

Danksagung 30

Interessenkonflikt $\quad 31$

$\begin{array}{ll}\text { Literatur } & 31\end{array}$

\section{Einleitung}

"When you hear hoofbeats behind you, think of horses, not zebras."

Theodore Woodward (1914-2005) .

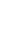

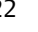
5 (5 ,

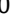
11

Dr. Theodore Woodward prägte diesen Merkspruch Ende der 1940er Jahre, um seinen Medizinstudenten an der University of Maryland zu vermitteln, bei einer bestimmten Symptomkonstellation zuerst an häufige und naheliegende Ursachen zu denken, bevor seltene Differenzialdiagnosen erwogen werden. Dementsprechend werden insbesondere im angloamerikanischen Sprachraum seltene Erkrankungen häufig als «Zebras» bezeichnet [1]. Für den einzelnen Patienten ist es irrelevant, ob er an einer häufigen oder einer seltenen Krankheit leidet. Für ihn zählt, dass er so zügig wie möglich die korrekte Diagnose und eine wirksame Therapie erhält. Daher sollten wir als Ärzte auch mit den seltenen Ursachen hinter häufigen Symptomen vertraut sein, d. h. bei Hufgeklapper an Pferde und Zebras denken. Besonders schwierig ist dies, wenn es sich dabei um das Leitsymptom «Schwindel» handelt. 


\subsection{Seltene Erkrankungen des vestibulären Laby- rinths}

\subsubsection{Probleme}

Schwindel ist mit einer geschätzten Ein-Jahres-Prävalenz zwischen 15 und $20 \%$ [2] eine der häufigsten Ursachen für einen Arztbesuch. Dahinter können sich aber viele verschiedene und insbesondere seltene Ursachen verbergen. Der vorliegende Beitrag fokussiert sich dabei auf diejenigen "Zebras», die wir als HNO-Ärzte in jedem Fall erkennen sollten - die seltenen Erkrankungen des vestibulären Labyrinths.

Bereits die Definition des Begriffes «seltene Krankheit» ist hier problematisch. Für viele vestibuläre Erkrankungen gibt es keine (z.B. Vestibularisparoxysmie) [3] oder weit divergierende Angaben über Inzidenz und Prävalenz (z. B. Neuritis vestibularis, M. Menière) [2]. Dementsprechend hilft der Blick in Datenbanken über seltene Erkrankungen (z. B. Orphanet) [4] nur bedingt weiter. Gemäss der Definition einer seltenen Erkrankung in der Europäischen Union mit höchstens 5 Betroffenen pro 100000 Einwohnern der Gesamtbevölkerung sind in diesen Datenbanken Krankheiten als «selten» deklariert, die uns als HNO-Ärzten eher häufig begegnen, wie z. B. der akute sensorineurale Hörverlust («Hörsturz») (ORPHAcode: 90059) oder das Ramsay-Hunt-Syndrom (ORPHAcode: 3020). Andererseits sind seltene, aber wichtige Differenzialdiagnosen peripher-vestibulärer Syndrome, wie z. B. die Vestibularisparoxysmie oder das intralabyrinthäre Schwannom, dort gar nicht aufgeführt.

\subsubsection{Lösungsansätze}

Erfreulicherweise gibt es einige Entwicklungen in der vestibulären Medizin, die das Erkennen von «Zebras» in der täglichen klinischen Praxis erleichtern. Hierzu gehört das im Jahr 2006 von der BárányGesellschaft begonnene Projekt zur Internationalen Klassifikation vestibulärer Erkrankungen (International Classification of Vestibular Disorders, ICVD) [5]. Die Ergebnisse werden laufend aktualisiert und sind im Internetauftritt des Journal of Vestibular Research frei zugänglich [6].

Weiterhin haben sich die diagnostischen Optionen bei Erkrankungen des Labyrinths in den letzten 20 Jahren deutlich verbessert. So stehen heute mit dem Video-Kopf-Impuls-Test (video head impulse test, vHIT)[7] und den vestibulär evozierten myogenen Potenzialen (VEMPs) [8-12] Methoden für eine spezifische, seitengetrennte, reproduzierbare, quantifizierbare und zeitsparende Diagnostik aller 5 vestibulären Rezeptororgane und ihrer Afferenzen zur Verfügung («Fünf-Rezeptor-Diagnostik») [13-15]. Während die okulären VEMPs (oVEMPs) überwiegend ein Mass für die kontralaterale Utrikulusfunktion darstellen, sind die cervicalen VEMPs (cVEMPs) überwiegend ein Indikator für die ipsilaterale Sakkulusfunktion [16]. Die Spezifität von o- und cVEMPs für Utrikulus und Sakkulus ist zwar nicht 100-prozentig (u.a. da im sog. Voit'schen Nerven 10\% der Sakkulusafferenzen über den N. vestibularis sup. laufen $[17,18]$ ), hat sich aber in zahlreichen klinischen Studien als suffizient für die klinische Differenzierung zwischen Utrikulus- und Sakkulus-Funktionsstörungen erwiesen [12].

Handliche und tragbare Diagnostikgeräte können am Patientenbett und in der Notaufnahme eingesetzt werden [19, 20]. Dadurch können heutzutage auch diejenigen seltenen vestibulären Erkrankungen flächendeckend erkannt werden, deren Diagnose bis vor einigen Jahren wenigen spezialisierten Zentren mit aufwändigen Apparaturen vorbehalten war. Dank Smartphone-Kameras oder «DizzyCam» können sogar die Patienten selbst ihre Nystagmen während episodisch auftretender Schwindelsyndrome aufzeichnen [21, 22]. Im Einzelfall kann dies den entscheidenden differenzialdiagnostischen Hinweis für eine bestimmte Erkrankung liefern, insgesamt wird so die Charakterisierung episodischer vestibulärer Syndrome weiter verbessert.

Die Weiterentwicklungen in der neurophysiologischen vestibulären Topodiagnostik werden durch Fortschritte in der Bildgebung der Schädelbasis ergänzt [23, 24]. Dies hat unmittelbare Auswirkungen auf die «Seltenheit» einer Erkrankung. Viele Patienten mit einem intralabyrinthären Schwannom haben vor 20 Jahren sicher auch deswegen die Diagnose eines Hörsturzes oder eines M. Menière erhalten, weil neben der zu geringen Aufmerksamkeit gegenüber dieser sehr seltenen Erkrankung die Auflösung des MRT für die Darstellung kleiner Tumoren zu schlecht war (siehe Kapitel 3.4.1).

Ein weiterer Meilenstein war die Schaffung validierter Diagnosealgorithmen für die klinische Praxis, welche eine zuverlässige Identifikation potentiell gefährlicher Ursachen vestibulärer Syndrome erlauben. So erkennen die klinisch orientierten «H.I.N.T.S. to I.N.F.A.R.C.T.» (H.I.N.T.S. = Head Impulse, Nystagmus, Test of $\boldsymbol{S}$ kew; I.N.F.A.R.C.T. = Impulse Negative, Fast Alternating (Nystagmus), Refixation on Cover Test) einen Hirnstamm- oder Kleinhirninfarkt als Ursache eines anhaltenden, akuten vestibulären Syndroms (AVS) zuverlässiger als ein frühes diffusionsgewichtetes (DWI) MRT ( <48 h nach Symptombeginn) oder kardiovaskuläre Risiko-Scores (z. B. $\mathrm{ABCD}^{2}=$ age, blood pressure, clinical features, duration, diabetes [25]) [26-31].

\section{2 «Gebrauchsanweisung» für diesen Beitrag}

Vor diesem Hintergrund soll der vorliegende Artikel keine erschöpfende, lexikalische Aufzählung seltener vestibulärer Krankheiten sein. Er soll vielmehr dem klinisch tätigen HNO-Arzt eine Entscheidungshilfe sein, wenn sich die Symptome und Befunde eines Patienten nicht durch die häufigen vestibulären Erkrankungen unseres Fachgebietes erklären lassen. Für die bessere Handhabung im klinischen Alltag sind die Erkrankungen dabei nicht nach Ätiologie geordnet, sondern nach ihrer klinischen Präsentation in Form eines akuten (AVS), episodischen (EVS) oder chronischen vestibulären Syndroms (CVS), welches jeweils spontan oder mit Triggern auftreten kann [28, 32]. Im Mittelpunkt stehen dabei immer folgende Fragen:

- Bei welcher Symptom- und Befundkonstellation sollte ich an eine seltene Erkrankung («Zebra») denken?

- Was sind «red flags» für eine potentiell gefährliche Ursache also einen "Wolf im Schafspelz»?

- Welche diagnostischen Massnahmen sind zielführend, um das eine vom anderen zu unterscheiden?

In diesem Sinne hoffe ich, dass der folgende Text für Sie zu einem Vademecum im «Zoo» des vestibulären Labyrinths wird - mit all seinen Zebras, Chamäleons und Wölfen im Schafspelz. 


\section{Akute vestibuläre Syndrome}

Das akute vestibuläre Syndrom (AVS) ist durch folgende Charakteristika gekennzeichnet $[5,28,32]$ :

- plötzlich aufgetretener Schwindel/Gleichgewichtsstörung (im Sinne von «vertigo», «dizziness» und/oder «unsteadiness» nach ICVD) [33]

- Dauer: mindestens 24 Stunden

- Symptome und Befunde einer neu aufgetretenen, anhaltenden vestibulären Funktionsstörung (z. B. Erbrechen, Nystagmus, Fallneigung, Gangunsicherheit).

\subsection{Akute einseitige peripher-vestibuläre Unterfunk- tion}

\subsubsection{Neuritis vestibularis inferior}

Während eine akute unilaterale Vestibulopathie (AUVP) in den meisten Fällen den N. vestibularis sup. [34] bzw. die von ihm versorgten Rezeptororgane betrifft (horizontaler Bogengang in 97,7\% der Fälle, anteriorer Bogengang: 90,7\%, Utrikulus: 72,1\%) [35], ist die Neuritis vestibularis inf. ein echter «Kolibri». Eine isolierte Unterfunktion des unteren Nervenastes und seiner Endorgane (posteriorer Bogengang und/oder Sakkulus) wird nur bei 1,2 bis $5 \%$ aller Patienten mit AUVP beobachtet [36-43]. Für das seltene Auftreten wird der unterschiedliche Verlauf der superioren und inferioren Anteile des Gleichgewichtsnerven verantwortlich gemacht. Der knöcherne Kanal des N. vestibularis sup. ist enger und ca. sieben Mal länger als der des unteren Nervenastes. Damit ist der obere Gleichgewichtsnerv anfälliger für Läsionen durch Druck bzw. Schwellung, wie sie durch eine entzündliche Reaktion (z. B. im Rahmen einer Reaktivierung von Herpes-simplex-Viren) ausgelöst werden $[44,45]$.

Gemäss des ersten Ewald'schen Gesetzes [46] zeigt sich bei Patienten mit Neuritis vestibularis inf. typischerweise ein Ausfallnystagmus in der Ebene des betroffenen posterioren Bogenganges, d. h. ein rotatorischer Nystagmus zum gesunden Ohr mit Downbeat-Komponente, welcher sich als peripher-vestibulärer Nystagmus durch Fixation supprimieren lässt ( $\bullet$ Abb. 1a) [47-49]. Da dieser Nystagmus durch eine Inhibition des posterioren Bogenganges ausgelöst wird, schlägt er in der gleichen Ebene - aber in genau entgegengesetzter Richtung - wie der Lagerungsnystagmus beim benignen paroxysmalen Lagerungsschwindel des ipsilateralen posterioren Bogenganges ( $\mathrm{p}$-BPLS), welcher durch eine Exzitation dieses Kanals entsteht $(\vee$ Abb. 1a) [50].

In der Fünf-Rezeptor-Diagnostik demarkieren sich ein reduzierter Gain mit Korrektursakkaden für den posterioren Bogengang im vHIT und/oder im Seitenvergleich reduzierte Amplituden der cVEMPs (Unterfunktion des ipsilateralen Sakkulus). In der Studie von Taylor et al. 2016 [35] waren bei einem Drittel der Patienten nicht beide Endorgane gleichzeitig betroffen. Da bei der isolierten Neuritis vestibularis inf. der superiore Nervenanteil ausgespart bleibt, fallen die Ergebnisse der kalorischen Prüfung (horizontaler Bogengang), des VHIT für die anterioren und horizontalen Bogenänge und der oVEMPs (Indikator für Utrikulusfunktion) für die betroffene Seite normal aus $[13,40,42,51]$.

\subsubsection{Akute einseitige Unterfunktion der Otolithenorgane}

Ein akutes vestibuläres Syndrom kann auch isoliert die Otolithenorgane betreffen. Über die genaue Inzidenz dieses Krankheitsbildes ist bislang nichts bekannt, da die Fünf-Rezeptor-Diagnostik mit vHIT, c- und oVEMPs erst seit einigen Jahren regulär zur Diagnose akuter vestibulärer Syndrome eingesetzt wird [52].

Leitsymptome bei einer akuten einseitigen Funktionsminderung der Otolithenorgane sind Schwank- oder Liftschwindel, das Gefühl, von hinten oder von der Seite "gestossen zu werden», Gangunsicherheit, Fallneigung bis hin zu Stürzen und starke Übelkeit bis zum Erbrechen [52, 53]. Allerdings berichten Patienten mit einer isolierten Unterfunktion der Otolithenorgane auch von Drehschwindel $[54,55]$. Im Falle einer akuten einseitigen Unterfunktion des Utrikulus kann gelegentlich ein überwiegend horizontaler Nystagmus zur gesunden Seite mit Fixationssuppression beobachtet werden - trotz intakter Funktion der Bogengänge in der kalorischen Prüfung und im vHIT [56, 57].

Diese auf den ersten Blick ungewöhnliche Beobachtung lässt sich dadurch erklären, dass etwa die Hälfte der sekundären vestibulären Neurone im Vestibulariskerngebiet sowohl afferente Informationen von primären vestibulären Neuronen der Bogengänge als auch der Otolithenorgane erhalten (sog. Konvergenzneurone) [58-62]. Eine Tonusdifferenz zwischen den sekundären vestibulä-
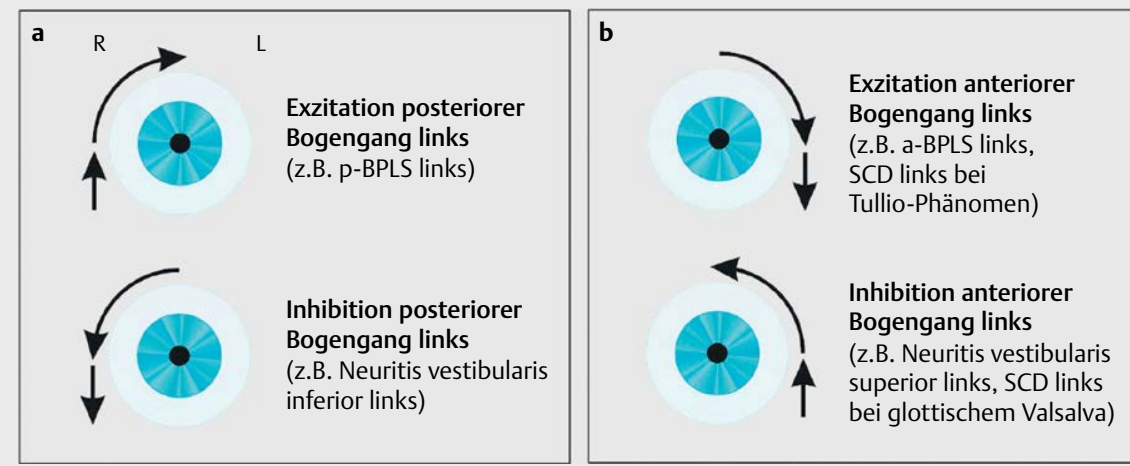

- Abb. 1 Nystagmusmuster bei Exzitation und Inhibition des linken a posterioren und $\mathbf{b}$ anterioren Bogenganges gemäss der Ewald'schen Gesetze [46] und der Studien von Cohen [47]. Details siehe Kapitel 2.1.1 und 3.2.1 bis 3.2.4. Abkürzungen: $a=$ anterior, BPLS= benigner paroxysmaler Lagerungsschwindel, $\mathrm{p}=$ posterior, $\mathrm{SCD}=$ Dehiszenz des superioren (= anterioren) Bogenganges (superior canal dehiscence). 
ren Neuronen im rechten und linken Vestibulariskerngebiet resultiert letztendlich in einem Spontannystagmus zur Seite des höheren Tonus [63], egal ob die Tonusdifferenz durch einen einseitigen Ausfall der Bogengangs- oder der Otolithenafferenzen zustande kommt. Da es sich hierbei um einen peripheren Nystagmus handelt, lässt er sich durch Fixation supprimieren [52, 57].

\section{Merke}

Auch eine einseitige Unterfunktion der Otolithenorgane kann sich mit Drehschwindel und einem Spontannystagmus vom peripheren Typ manifestieren.

Damit ist die akute einseitige Unterfunktion der Otolithenorgane ein anschauliches Beispiel dafür, dass die Symptomqualität des Schwindels nicht immer einen Rückschluss auf den Ort der vestibulären Läsion erlaubt [28]. Daher sollte in jedem Fall eines akuten vestibulären Syndroms eine dezidierte klinisch-neurootologische Untersuchung mit dem «H.I.N.T.S. plus» Algorithmus (Kap. 1.1.2 und 2.2.1) - optimal ergänzt durch eine apparative Fünf-Rezeptor-Diagnostik - erfolgen, um das vestibuläre Defizit möglichst genau zu lokalisieren (peripher vs. zentral, Bogengänge vs. Otolithenorgane).

Isolierte Funktionsstörungen der Otolithenorgane bei intakter Funktion der Bogengänge finden sich häufig nach Kopfanprall- und Explosionstraumata. Dies wird auf die erhöhte Empfindlichkeit der Otolithenorgane gegenüber mechanischen Reizen in Vergleich zu den Bogengängen zurückgeführt [64]. In einer Studie wurde bei Patienten mit Schwindel ("dizziness») nach Schädel-Hirn-Trauma in 72 \% der Fälle ein einseitiges Otolithendefizit diagnostiziert (pathologische VEMPs, verkippte subjektive visuelle Vertikale), während die Patienten ohne Schwindel nach vergleichbarem Trauma nur in etwa $20 \%$ auffällige Befunde in der Funktionsprüfung der Otolithenorgane aufwiesen [65]. Auch nach Explosionstraumata finden sich gehäuft reduzierte c- und oVEMP-Antworten [66].

\subsubsection{Therapie}

Die Therapie der akuten Neuritis vestibularis inferior und der isolierten akuten einseitigen Otolithenfunktionsstörung erfolgt wie bei anderen Fällen einer AUVP. Neben der Gabe von Glukokortikoiden ist der frühe Beginn eines massgeschneiderten vestibulären Trainings entscheidend [67, 68]. Basierend auf den Ergebnissen der Fünf-Rezeptor-Diagnostik kann hier die zentrale Kompensation der jeweils ausgefallenen peripher-vestibulären Funktion gezielt gefördert werden.

\subsection{Akute einseitige audiovestibuläre Funktionsstö- rung}

Diese Symptomkonstellation wird traditionell als "Labyrinthitis» bezeichnet. Während dieser Begriff eine entzündliche Erkrankung des Innenohrs impliziert, legen aktuelle Studien nahe, dass sich dahinter auch ein Labyrinthinfarkt oder eine Labyrinthblutung mit potentiell gefährlichen Folgen verbergen kann [31].

\subsubsection{Labyrinthinfarkt}

Die A. labyrinthi entspringt in ca. $80 \%$ der Fälle aus der A. inferior anterior cerebelli (AICA), seltener direkt aus der A. vertebralis/basilaris (15-20\%) oder der A. posterior inferior cerebelli (PICA, 2-3\%)
$[31,69]$. Da es sich bei der A. labyrinthi um einen Endast mit nur wenigen Kollateralen handelt, ist das Innenohr besonders anfällig für Ischämien. Diese können abhängig von der Lokalisation des Gefässverschlusses das gesamte Innenohr oder Teile davon betreffen [70] (siehe $[71,72]$ für eine Darstellung der einzelnen Äste und ihrer Versorgungsgebiete). Insbesondere die Kombination aus einer Funktionsstörung des posterioren Bogenganges und einer sensorineuralen (cochleären) Schwerhörigkeit ist verdächtig auf das Vorliegen einer vaskulären Läsion, da beide Rezeptororgane von der A. cochlearis communis bzw. der A. vestibulo-cochlearis versorgt werden [72].

Das Gefährliche am akuten Labyrinthinfarkt sind nicht so sehr die Hörminderung oder der Schwindel, sondern die mögliche Progression zu einem Hirnstamm- oder Kleinhirninfarkt [70, 71]. In mehreren retro- und prospektiven Studien berichteten zwischen 8 und $30 \%$ der Patienten mit einem MR-tomografisch nachgewiesenen AICA-Infarkt über einen einseitigen Hörverlust mit Schwindel innerhalb von 30 Tagen vor dem Auftreten des Hirninfarktes $[69,70,73]$. Gerade bei Patienten mit kardiovaskulären Risikofaktoren (z. B. ABCD ${ }^{2}$ Score $\geq 4$ ) sollte daher beim erstmaligen Auftreten eines akuten, anhaltenden vestibulären Syndroms (d. h. Dauer > 24h) mit einseitiger Hörminderung eher an eine vaskuläre Läsion (Labyrinthinfarkt) als an eine entzündliche Ursache (Labyrinthitis) gedacht werden [71, 74, 75].

Die Diagnosestellung ist allerdings durch mehrere Faktoren erschwert. Erstens steht derzeit keine «beweisende» Bildgebung für einen isolierten Labyrinthinfarkt ohne Hirnstamm- oder Kleinhirnbeteiligung zur Verfügung $[70,71,75,76]$. So gibt es bislang nur wenige Fallberichte, bei denen der Nachweis einer Diffusionsrestriktion im Bereich des $\mathrm{N}$. vestibulocochlearis mittels hochauflösendem DWI-MRT des Schläfenbeines gelang (1,4 mm anstatt wie üblich $5 \mathrm{~mm}$ Schichtdicke) $[78,87]$. Eine Diffusionsminderung im Bereich des Innenohres selbst konnte bislang nicht dargestellt werden [28]. Durch die Anwendung von 3D-FLAIR-Sequenzen (FLAIR = fluid attenuated inversion recovery) wird zwar die Sensitivität des MRT für Innenohrpathologien im Vergleich zur T1-Wichtung erhöht [79]. Allerdings ist mit den aktuellen Methoden noch keine genauere Differenzierung möglich, ob eine Anreicherung von Gadolinium im Innenohr in der FLAIR-Sequenz entzündlich oder vaskulär bedingt ist (vaskulär: [80, 81]; entzündlich: [82]).

Zweitens führt der isolierte Labyrinthinfarkt nicht zu den klassischen «H.I.N.T.S. to I.N.F.A.R.C.T», da es sich ja um eine peripherund nicht um eine zentral-vestibuläre Störung handelt [31]. Die klassischen «H.I.N.T.S» geben Hinweise auf den Ort der Schädigung (zentral vs. peripher), und nicht auf die Ursache (entzündlich vs. vaskulär). Um dennoch der Bedeutung des Labyrinthinfarktes als möglichem Vorboten eines Hirninfarktes Rechnung zu tragen, wurde im aktualisierten «H.I.N.T.S. plus» Paradigma die akute einseitige Hörminderung bei AVS als zusätzliche «red flag» für einen Infarkt hinzugefügt [27]. In einer Querschnittsstudie an Patienten mit einem akut-vestibulärem Syndrom und erhöhtem Risiko für einen Schlaganfall wurde mit den «H.I.N.T.S. plus» ein zugrundeliegender Infarkt mit einer Sensitivität von 99,2\% und einer Spezifität von $97,0 \%$ erkannt, während Sensitivität und Spezifität für einen $A B C D^{2}$ score $\geq 4$ nur bei jeweils ca. $60 \%$ lagen. In den ersten $48 \mathrm{~h}$ nach Symptombeginn waren die «H.I.N.T.S. plus» sogar dem DWIMRT in der Sensitivität überlegen, da sich insbesondere kleine In- 
farkte des hinteren Stromgebietes häufig erst nach 48-72 h im DWI-MRT demarkieren [31,83].

Daraus resultiert auch die Empfehlung von Newman-Toker et al. [27], Patienten mit positiven «H.I.N.T.S. plus», bei denen keine Indikation für eine Lyse besteht bzw. das Lysefenster überschritten ist, zunächst für $48 \mathrm{~h}$ zu überwachen und dann erst ein DWI-MRT durchzuführen. In jedem Fall sollte unmittelbar ein Neurologe mit hinzugezogen werden, unter dessen Federführung die weitere neurovaskuläre Diagnostik, Therapie und ggf. Sekundärprophylaxe erfolgt. Details hierzu finden sich bei $[32,84]$.

\section{Merke}

Eine erstmals aufgetretene, anhaltende, akute cochleovestibuläre Störung gilt bis zum Beweis des Gegenteils als verdächtig auf einen Labyrinthinfarkt. Ein negatives diffusionsgewichtetes cMRT in den ersten $48 \mathrm{~h}$ nach Symptombeginn schliesst einen AICA- oder PICA-Infarkt nicht aus.

Ein Labyrinthinfarkt kann auch im Rahmen einer Basilaris- oder Vertebralisthrombose auftreten, entweder durch eine arterio-arterielle Embolie oder durch eine Minderperfusion der A. labyrinthi aufgrund der Thrombose $[85,86]$. Einen weiteren Sonderfall stellt ein Labyrinthinfarkt auf dem Boden einer Vertebralisdissektion dar. Letztere kann spontan oder nach einem entsprechenden Trauma (z. B. Auffahrunfall, Manipulation an der HWS) auftreten und sollte insbesondere bei jüngeren Patienten ohne kardiovaskuläre Risikofaktoren bedacht werden [81, 87-89].

\subsubsection{Labyrinthblutung}

Neben einer Ischämie führen auch Einblutungen in das Labyrinth zu der klinischen Präsentation eines akuten vestibulären Syndroms mit einseitiger Hörminderung. Als mögliche Ursachen wurden Traumata, Gerinnungsstörungen (z. B. im Rahmen einer Leukämie), die Einnahme von oralen Antikoagulantien, Einblutungen in einen Tumor des endolymphatischen Sacks (siehe Kapitel 3.4.2) oder eine superfizielle Siderose (siehe Kapitel 4.1.3.2.2) berichtet. Selten treten Labyrinthblutungen auch spontan auf [90-92]. Kürzlich wurde erstmals ein Fall einer bilateralen Labyrinthblutung bei einer 18-jährigen Patientin mit SARS-CoV-2-Infektion (severe acute respiratory syndrome coronavirus 2) beschrieben [93].

Im Gegensatz zur Labyrinthischämie lässt sich die Blutung bereits in der nativen T1- und FLAIR-Sequenz des Schläfenbein-MRTs als hyperintense Läsion ohne weitere Verstärkung durch Kontrastmittelgabe darstellen [82, 92]. Bei Patienten mit spontaner Labyrinthblutung sollte eine Gerinnungsstörung als mögliche Ursache abgeklärt werden. Neben der Therapie der Grunderkrankung ist auch eine systemische oder intratympanale Gabe von Glucocorticoiden zu erwägen. Hier wurde in einzelnen Fallberichten eine partielle Erholung der Innenohrfunktion beschrieben [90]. a

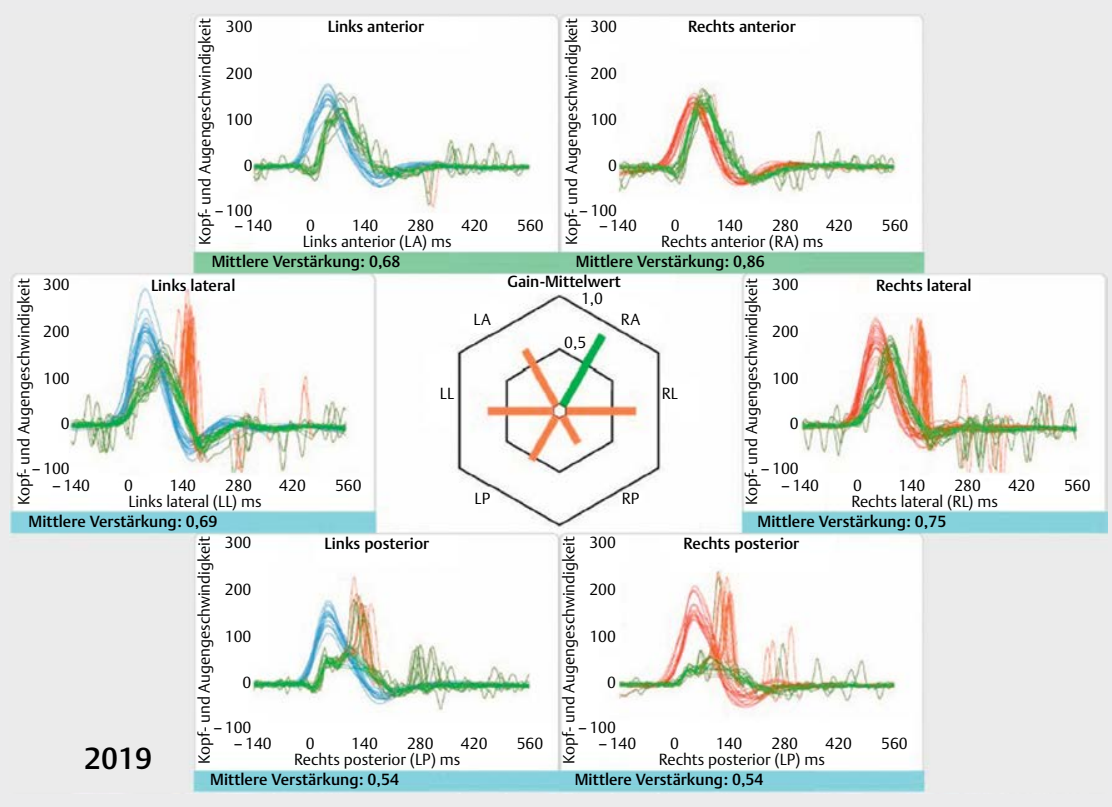

b $\quad 125 \quad 250 \quad 500 \quad 1 \mathrm{k} \quad 2 \mathrm{k} \quad 4 \mathrm{k} \quad 8 \mathrm{k} \quad 12 \mathrm{k}$

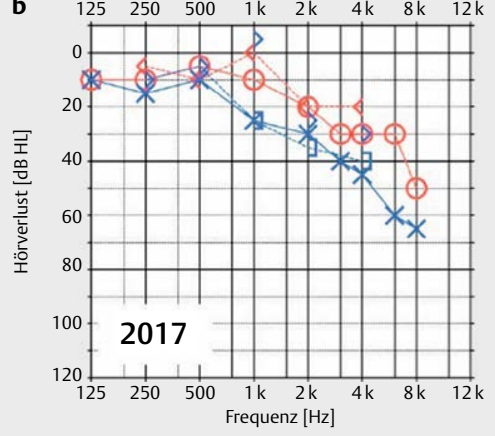

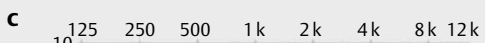

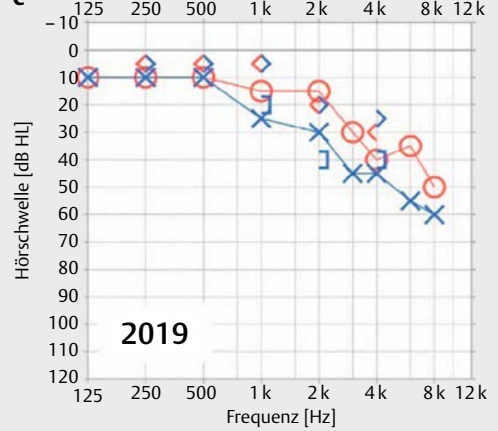

- Abb. 2 Gentamicin-induzierte bilaterale Vestibulopathie (BVP). Der 59-jährige Patient wurde wegen einer Endokarditits nach Aortenklappenersatz von Mai bis Juni 2019 intravenös mit Gentamicin behandelt. Drei Wochen nach Abschluss der Therapie beklagte er erstmals Oszillopsien und Gangunsicherheit. Ruheschwindel und subjektive Hörminderung wurden verneint, es lag kein Spontannystagmus vor. a Im Video-Kopf-Impuls-Test (vHIT) zeigte sich das typische Bild einer Aminoglykosid-assoziierten BVP mit reduziertem Gain und Korrektursakkaden der horizontalen (=lateralen) und posterioren Bogengänge bds. bei erhaltener Funktion der anterioren Bogengänge bds. (normaler Gain, keine Korrektursakkaden). Details siehe Kapitel 4.1.3. b und c Im Vergleich zum Vorbefund von 2017 mit sensorineuraler hochtonbetonter Schwerhörigkeit bds. demarkierte sich im Reintonaudiogramm nach Gentamicingabe kein zusätzlicher Hörverlust. 


\subsubsection{Labyrinthitis}

Von einer «(Neuro-)Labyrinthitis» sollte tatsächlich nur dann gesprochen werden, wenn Hinweise für eine entzündliche Erkrankung des Mittel-/Innenohres (z. B. Otitis media) bzw. seiner Afferenzen (z.B. Meningitis) vorliegen, welche die anhaltende, akute audiovestibuläre Funktionsminderung hinreichend erklären können [31]. Neben dem klassischen Fall einer akuten Otitis media mit Innenohrbeteiligung kann dies eine Infektion mit neurotropen Viren (z. B. Herpes zoster, Masern, Mumps, CMV, EBV, HIV) oder Bakterien (z. B. Borrelien) sein [82,94]. Daher sollte insbesondere auf entsprechende Effloreszenzen im HNO-Bereich und am ganzen Körper geachtet werden. Bislang liegen bis auf einzelne Fallberichte ohne dezidierte neurootologische Untersuchungen (z. B. [93, 96]) keine systematischen Analysen über die Auswirkungen von SARS-CoV-2 auf das vestibuläre Labyrinth vor (siehe auch Kapitel 2.2.2). Der neurotrope Charakter des Virus [97] und das Auftreten von akutem sensorineuralen Hörverlust bei Patienten mit COVID-19-Erkrankung (coronavirus disease 2019) legen jedoch auch eine vestibuläre Beteiligung nahe, was insbesondere in klinischen Studien zu Langzeitfolgen der Erkrankung berücksichtigt werden sollte [98-102].

Sollten neben einem akuten audiovestibulären Defizit simultan oder sequentiell noch andere Hirnnervenparesen auftreten, so ist differenzialdiagnostisch an eine basale Meningitis zu denken. Diese kann - auch im 21. Jahrhundert - insbesondere im Rahmen einer Tuberkulose oder einer Syphilis beobachtet werden [103]. Auch eine Meningeosis carcinomatosa oder ein ZNS-Lymphom können zu isolierten Hirnnervenausfällen führen. Für die weitere Diagnostik (inklusive Lumbalpunktion und Bildgebung) und Therapie sollten zügig die Kollegen der Neurologie - idealerweise mit Erfahrung in Neuroimmunologie/-infektiologie - hinzugezogen werden.

\subsection{Akute beidseitige peripher-vestibuläre Funk- tionsstörung}

Eine akute simultane Funktionsstörung beider Gleichgewichtsorgane bzw. deren Afferenzen ist sehr selten und meistens toxisch (z. B. Aminoglykoside), traumatisch (z. B. beidseitige Schläfenbeinfraktur) oder infektiös (z. B. basale Meningitis) bedingt (siehe hierzu die Fallbeispiele in $\mathbf{A} \mathbf{A b b} . \mathbf{2}$ und $\mathbf{3}$ ). Auch eine simultane Ischämie beider Aa. labyrinthi (z. B. im Rahmen einer Megadolichobasilaris) kann zu einem akuten Ausfall der Labyrinthfunktion beidseits führen [104]. Eine simultan auftretende Neuritis vestibularis beidseits ist ein echter vestibulärer "Kolibri», der bisher nur in 2 Kasuistiken beschrieben wurde $[105,106]$.

Ätiologie, Symptomatik, klinische und apparative Untersuchungsbefunde der bilateralen Vestibulopathie (BVP) werden in Kapitel 4 detailliert behandelt, da es sich meist um ein chronisches vestibuläres Syndrom handelt. Die akute Form dieses Krankheitsbildes wird aufgrund der für ein AVS ungewöhnlichen Symptomatik in der klinischen Praxis leider häufig übersehen, deswegen wird die akute BVP aus systematischen Gründen an dieser Stelle gesondert erwähnt.

Im Gegensatz zum akuten einseitigen vestibulären Syndrom beklagen Patienten mit einem akuten simultanen Verlust der peripher-vestibulären Funktion beidseits in der Regel keinen gerichteten Dreh- oder Schwankschwindel in Ruhe und zeigen auch keinen Spontannystagmus, da keine Tonusdifferenz zwischen rechtem und linkem Vestibulariskerngebiet vorliegt. Die Leitsymptome sind eine ausgeprägte Gleichgewichtsstörung im Stehen und Gehen sowie Oszillopsien (Details in Kapitel 4.1.1). Die Diagnosestellung ist mit einfachen Mitteln am Patientenbett möglich. Im klinischen KopfImpuls-Test für die horizontalen Bogengänge zeigt sich beidseits eine Einstellsakkade, im Romberg-Test mit geschlossenen Augen besteht eine ausgeprägte Fallneigung nach hinten, und die Untersuchung mit der Sehtest-Tafel ergibt eine reduzierte dynamische Sehschärfe beidseits [107]. Durch die apparative Untersuchung mit vHIT, c- und oVEMPs lässt sich das Ausmass der Schädigung in den vestibulären Endorganen quantifizieren und im Verlauf verfolgen ( Abb. 2 und 3, Details in Kapitel 4.1.2).

\section{Merke}

Patienten mit einer akuten bilateralen Vestibulopathie zeigen einen beidseits positiven Kopf-Impuls-Test, aber meist keinen Spontannystagmus.

\section{Episodische vestibuläre Syndrome}

Das EVS ist durch folgende Charakteristika gekennzeichnet $[5,28,32]$ :

- vorübergehender Schwindel/Gleichgewichtsstörung

- Dauer: Sekunden bis Stunden, selten Tage

- Symptome und Befunde einer vorübergehenden, kurz andauernden vestibulären Funktionsstörung (z. B. Übelkeit, Nystagmus, Sturz).

Da die Dauer des EVS eine wichtige Rolle bei der Differenzialdiagnose stellt, sind die folgenden seltenen Krankheitsbilder nach ansteigender Dauer der Attacken geordnet. Manche dieser Erkrankungen, insbesondere die Syndrome des dritten Fensters (Kapitel 3.2), stellen wahre «Chamäleons» dar, welche sich in Gestalt mehrerer episodischer vestibulärer Syndrome präsentieren können. Darauf wird bei den jeweiligen Krankheitsbildern hingewiesen.

\subsection{Spontaner episodischer Schwindel (Sekunden)}

\subsubsection{Vestibularisparoxysmie}

\subsubsection{Pathogenese}

Über die Prävalenz dieser seltenen vestibulären Erkrankung sind bislang keine Zahlen publiziert [3]. Als Ursache gilt ein Gefäss-Nerven-Konflikt im Kleinhirnbrückenwinkel. Ein permanenter Kontakt des N. vestibulocochlearis mit einer pulsierenden Gefässschlinge führt zu fokalen Demyelinisierungen der Nervenfasern. Neuronale Erregungen können nun direkt zwischen den demyelinisierten Axonen überspringen (ephaptische Übertragung), was letztendlich zu einer Hyperexzitation des N. VIII mit vestibulären und/oder audiologischen Symptomen führt - je nachdem, welcher Anteil des Nerven Kontakt mit der Gefässschlinge hat [108-111].

In einer MRT-Studie lag der Abstand des komprimierenden Gefässes vom Hirnstamm zwischen 0 und 10,8 mm [112], was dem zentralen Anteil des N. vestibulocochlearis entspricht. Dessen Myelinscheide wird von Oligodendrozyten gebildet und ist im Vergleich zur peripheren, von Schwann-Zellen produzierten, Myelinscheide besonders anfällig für fokale Demyelinisierungen $[111,113]$. Dabei handelte es sich in $75 \%$ der Fälle um die AICA, in jeweils $10 \%$ um die $A$. vertebralis oder eine Vene und in $5 \%$ um die PICA [112]. 

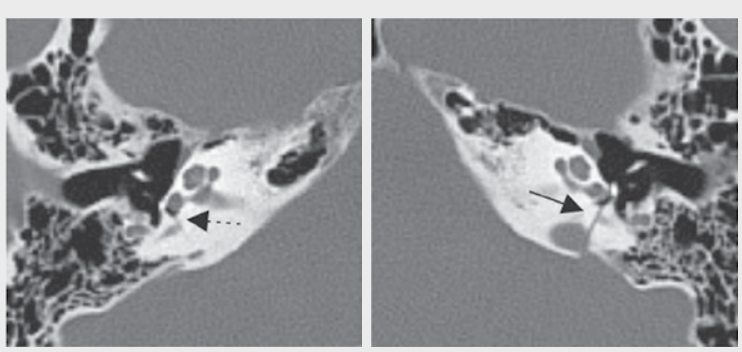

b
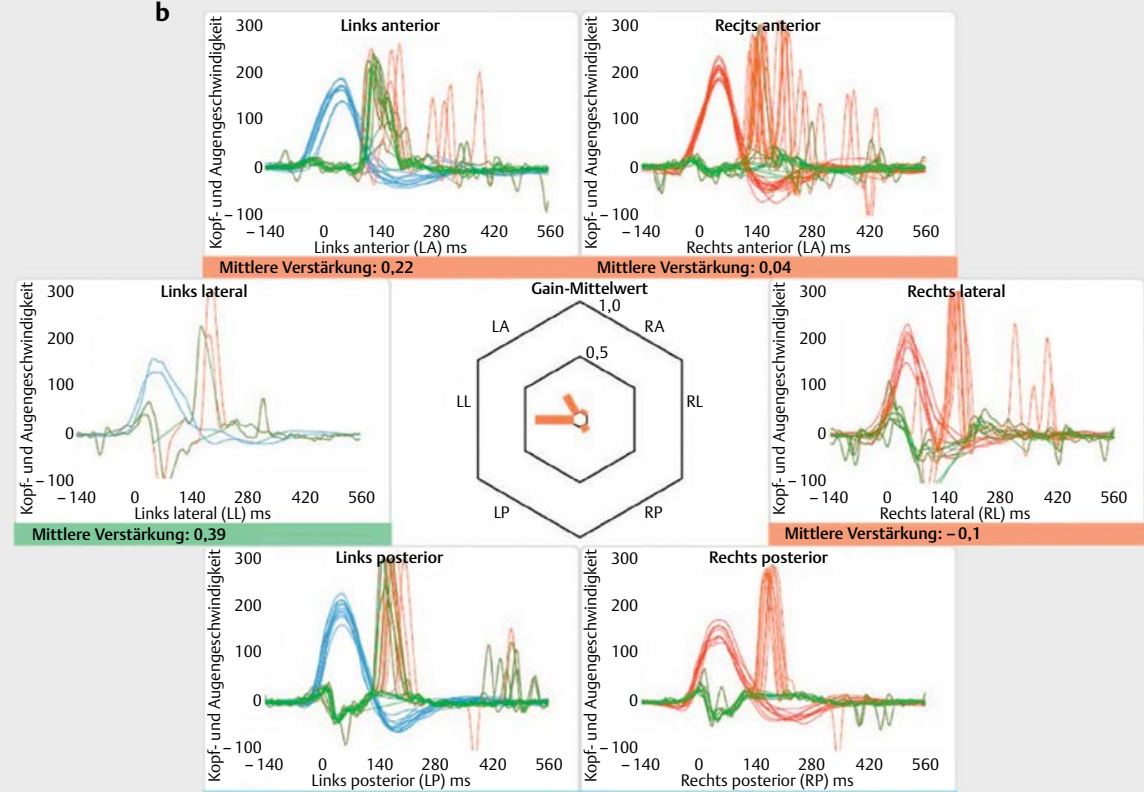

Mittlere Verstärkung: 0

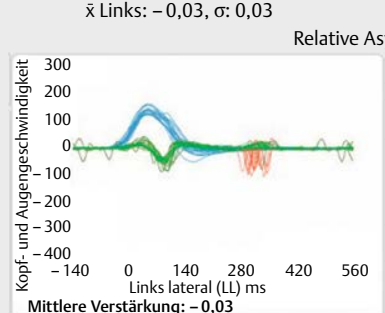
Mittlere Verstärkung: 0,07

- Rechts: $0,02, \sigma: 0,04$

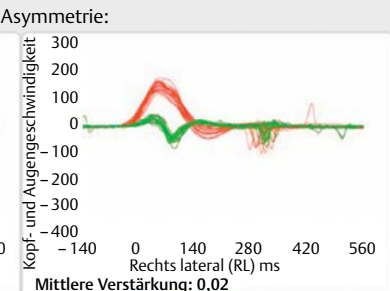

Abb. 3 Bilaterale Schläfenbeinquerfraktur mit akutem bilateralen Vestibularisausfall und beidseitiger Ertaubung nach Treppensturz. Der 63-jährige Patient beklagte keinen Schwindel, zeigte keinen Spontannystagmus, wies aber eine ausgeprägte Gangunsicherheit auf. a In den axialen Schichten des HRCT Schläfenbein demarkierte sich links klar eine Frakturlinie durch das Vestibulum (Pfeil), während rechts in diesem Bereich nur ein Haarriss (Pfeil mit gestrichelter Linie) zu sehen war. Auf beiden Seiten liess sich freie Luft im Vestibulum als Frakturzeichen nachweisen. b Bilaterale Vestibulopathie mit Beteiligung aller sechs Bogengänge: Die Kooperation des Patienten bei der Durchführung des Video-Kopf-Impuls-Testes (vHIT) war bei Surditas bds. erschwert (Artefakte bei der Messung des linken lateralen Bogenganges). Für alle 6 Bogengänge wurde ein deutlich erniedrigter Gain<0,25 mit ausgeprägten Korrekursakkaden („Overt“ Sakkaden) gemessen. c In der SHIMP-Messung (suppression of head impulses) der horizontalen (=lateralen) Bogengänge zeigten sich keine Sakkaden. Dies spricht für einen kompletten Ausfall des horizontalen vestibulo-okulären Reflexes beidseits (Details bei [418]). Okuläre und cervicale vestibulär evozierte myogene Potentiale (VEMPs) waren beidseits ausgefallen (nicht dargestellt).

\subsubsection{Symptome und diagnostische Kriterien}

Patienten mit Vestibularisparoxysmie (VP) berichten über blitzartige, nur einige Sekunden dauernde, stereotype Schwindelsensationen (meist Drehschwindel, aber auch Schwankschwindel und Fallneigung), welche in Extremfällen bis zu 100-mal am Tag auftreten können $[3,110]$. Meist ereignen sich diese Paroxysmen spontan, können aber auch durch bestimmte Kopfdrehungen ausgelöst werden. Je nach Beteiligung des Hörnerven werden auch entsprechende auditorische Sensationen zusammen mit oder unabhängig von den Schwindelattacken ausgelöst. Pathognomonisch ist hier ein stakkatoartiges Ohrgeräusch, welches bei den Patienten häufig Assoziationen mit dem Tippgeräusch einer Schreibmaschine weckt («Typewriter Tinnitus») [114]. 
In den diagnostischen Kriterien der Bárány-Gesellschaft werden eine "klinisch sichere» und eine "wahrscheinliche» Vestibularisparoxysmie unterschieden [3]. Neben der höheren Zahl (10 vs. 5) und der kürzeren Dauer ( $<1$ vs. $<5$ min) der stattgehabten Attacken wird für die sichere Form im Gegensatz zur wahrscheinlichen gefordert, dass eine Besserung der Symptomatik durch Therapie mit einem Natriumkanalblocker eingetreten ist (siehe unten).

Merke

Die Diagnose einer "sicheren» Vestibularisparoxysmie kann erst nach einem Therapieversuch mit einem

Natriumkanalblocker gestellt werden. Der Nachweis eines Gefäss-Nerven-Kontaktes im MRT ist für die Stellung der Diagnose nicht erforderlich.

\subsubsection{Untersuchungsbefunde}

Gelingt es dem Untersucher, eine der sehr kurzen Attacken zu beobachten, zeigt sich meist ein horizontal-rotatorischer Reiznystagmus in Richtung des betroffenen Ohres. Bei ca. 70\% der Patienten lässt sich durch dreiminütige Hyperventilation ein Nystagmus in Richtung des betroffenen Ohres auslösen [110]. Dabei handelt es sich i.d.R. nicht um die Provokation einer Attacke. Vielmehr wird durch die Hyperventilations-induzierte Alkalose die Ca ${ }^{2+}$-Konzentration in der Extrazellulärflüssigkeit reduziert, was die Exzitationsschwelle der demyelinisierten Axone weiter senkt [115].

Im freien Intervall wurde bei 30-40\% der Patienten eine milde (audio-)vestibuläre Funktionseinschränkung auf der betroffenen Seite beschrieben. Auch ein Nebeneinander von Befunden, welche für eine Überfunktion (z. B. Reiznystagmus) und eine Unterfunktion (z. B. Ausfallnystagmus, kalorische Untererregbarkeit, reduzierter Gain im vHIT, reduzierte VEMP-Amplitude) der betreffenden Seite sprechen, wurde beobachtet $[110,112]$.

\subsubsection{Bildgebung}

Der Gefäss-Nerven-Kontakt lässt sich am besten durch ein Dünnschicht-MRT des Kleinhirnbrückenwinkels $(\leq 0,7 \mathrm{~mm}$ ) in einer stark T2-gewichteten Sequenz (CISS = constructive interference in steady state oder FIESTA = fast imaging employing steady state aquisition) darstellen $[111,116]$. Ein Gefäss-Nerven-Kontakt liegt vor, wenn zwischen den hypointens dargestellten Nerven und Gefässen kein hyperintenser Liquor mehr zu sehen ist. Der Nachweis eines neurovaskulären Konfliktes im MRT weist zwar eine hohe Sensitivität von $95 \%$ und mehr für die Diagnose einer Vestibularisparoxysmie auf, die Spezifität betrug in einer Studie aber nur 65\% - oder anders ausgedrückt: bei 35 \% der Studienteilnehmer, welche keinerlei Symptome einer VP zeigten, liess sich auch ein Gefäss-NervenKontakt im MRT nachweisen [110,112].

\subsubsection{Differenzialdiagnosen}

Die Durchführung des kranialen und Schläfenbein-MRT erfolgt daher in erster Linie zur Abklärung möglicher "Zebras», welche die Symptomatik einer VP imitieren können, z. B. Arachnoidalzysten oder Tumoren des Kleinhirnbrückenwinkels [117-119]. Eine Megadolichobasilaris kann aufgrund ihres geschlängelten Verlaufes gleichzeitig Kompressionssyndrome mehrerer Hirnnerven auslösen [120]. Eine Erweiterung der A. basilaris oder vertebralis ist häu- fig mit arterieller Hypertonie assoziiert und birgt das Risiko für Hirnstamminfarkte [121]. Hier sind neben der symptomatischen Therapie des neurovaskulären Konfliktes (s. unten) eine konsequente Blutdrucksenkung und eine neurologische Vorstellung des Patienten erforderlich, um Folgeschäden zu vermeiden. Eine Bestrahlung im Kleinhirnbrückenwinkel kann durch eine Schädigung der Oligodendrozyten eine Demyelinisierung des achten Hirnnerven und somit Symptome einer VP auslösen, welche sich ebenfalls gut mit einem Natriumkanalblocker behandeln lassen [115].

\subsubsection{Therapie}

Eine Therapie mit Natriumkanalblockern (Carbamazepin 200-800 mg oder Oxcarbazepin 300-900 mg pro Tag) reduziert die ephaptischen Entladungen und führt innerhalb weniger Tage bis Wochen meist zu einer deutlichen Reduktion von Attackenfrequenz und schwere [110], wie in einer doppelblind randomisierten kontrollierten Studie für Oxcarbazepin gegen Placebo gezeigt wurde [122]. Allerdings brachen viele Patienten aufgrund von Nebenwirkungen die Studie vorzeitig ab. Eine besser verträgliche Alternative scheint der Natriumkanalblocker Lacosamid zu sein [123]. Auch Phenytoin oder Valproat können verwendet werden. Die mikrovaskuläre Dekompression des Nerven sollte für die seltenen Fällen vorbehalten sein, in denen eine Therapie mit Natriumkanalblockern nicht möglich oder erfolgreich ist [3].

\subsubsection{Tumarkin-Krisen}

Diese spontan auftretenden Sturzattacken («drop attacks») ohne Bewusstseinsverlust werden von ca. $10 \%$ der Patienten mit M. Menière berichtet und sind in der Regel nicht von Drehschwindel oder einer vegetativen Symptomatik begleitet. Die Betroffenen berichten über ein plötzliches Gefühl, «als ob sie von hinten gestossen werden» oder als ob ihnen «jemand die Beine wegzieht.» Die Attacken dauern nur wenige Sekunden, und nach dem Aufstehen können die Betroffenen wieder normal ihren Aktivitäten nachgehen. Aufgrund des plötzlichen Auftretens ohne Prodromi besteht jedoch eine hohe Verletzungsgefahr $[124,125]$.

Erstmals von Tumarkin 1936 als "otolithic catastrophes» beschrieben, werden diese Attacken auf eine plötzliche Stimulation der vorgeschädigten Otolithenorgane durch einen Endolymphhydrops zurückgeführt. Die abrupte Aktivierung der vestibulo-spinalen Bahnen resultiert schliesslich in einem plötzlichen Tonusverlust der Beine mit Sturz $[126,127]$. Für diese Hypothese sprechen aktuelle Ergebnisse aus der Innenohrbildgebung (vermehrter vestibulärer Endolymphhydrops bei Menière-Patienten mit Tumarkin-Krisen) und aus VEMP-Untersuchungen (Restfunktion des Utrikulus) $[53,124,125]$. Tumarkin-Krisen sprechen gut auf eine intratympanale Gentamicinapplikation an (Erfolgsraten von $80 \%$ ) [125], was durch die vestibulotoxische Wirkung von Aminoglykosiden auf Typ I vestibuläre Haarzellen zu erklären ist (siehe Kapitel 4.1.3.1.1).

\subsection{Lärm- und druckinduzierter episodischer Schwin- del (Sekunden) - Syndrome des dritten Fensters}

Bei diesen Erkrankungen liegt im knöchernen Labyrinth neben dem ovalen und dem runden Fenster noch ein zusätzliches "drittes» Fenster zum Mittelohr oder zum Schädelinneren vor, welches als "Locus minoris resistentiae» zu einer veränderten Fluiddynamik des 
Innenohrs mit charakteristischen Veränderungen der Hör- und Gleichgewichtsfunktion führt.

Ein drittes Fenster der otischen Kapsel kann entweder durch eine Erweiterung eines natürlichen neurovaskulären Foramens (z. B. innerer Gehörgang, Aqueductus vestibuli) oder durch eine neu aufgetretene knöcherne Dehiszenz (z. B. Bogengangsdehiszenz) bzw. Ausdünnung des Knochens («Near dehiscence») entstehen. Während die meisten dritten Fenster genau lokalisiert werden können, gibt es auch «diffuse» Öffnungen, welche insbesondere bei Knochenerkrankungen (z. B. M. Paget, Osteospongiose, Osteogenesis imperfecta, fibröse Dysplasie) auftreten können (siehe hierzu auch das Referat von Frau Dr. Weiss [128]). Bei letzteren kommt es insgesamt zu einer Abnahme des knöchernen Widerstandes der otischen Kapsel, ggf. auch in Verbindung mit mehreren Mikrofrakturen, welche in der Summe ein drittes Fenster ergeben können [129131]. Schliesslich ist noch zu beachten, dass entzündliche (z. B. Cholesteatom), infektiöse (z. B. Syphilis), neoplastische (z. B. multiples Myelom, Langerhanszell-Histiozytose, Sarkome) und vaskuläre (z. B. Paragangliome) destruktive Prozesse der lateralen Schädelbasis neben zahlreichen anderen Manifestationen auch zu knöchernen Dehiszenzen der otischen Kapsel führen können $[129,130,132]$. Hierzu finden sich ebenfalls weitere Details im Beitrag von Frau Dr. Weiss [128]

\subsubsection{Pathophysiologie und Leitsymptome}

Die Auswirkungen eines zusätzlichen dritten Fensters in der otischen Kapsel auf die Hör- und Gleichgewichtsfunktion wurden in Tier- und mathematischen Modellen eingehend untersucht [133138]. Im Wesentlichen lassen sich die klinischen Symptome und die elektrophysiologischen Befunde vier Gruppen zuordnen:

Druckinduzierter Schwindel Schwankungen des intrakraniellen Drucks (z. B. beim Niesen, Pressen, Husten, Heben schwerer Gegenstände) oder des Mittelohrdrucks (z. B. schnelle Höhenwechsel) werden aufgrund der defekten knöchernen Begrenzung am dritten Fenster direkt auf die Flüssigkeitsräume des Innenohres wei- tergeleitet ( $\triangleright$ Abb. 4). Wird dadurch ein unphysiologischer Endolymphstrom im Gleichgewichtsorgan ausgelöst (z. B. bei knöcherner Dehiszenz eines Bogenganges), resultiert eine kurzfristige Schwindelsensation in Verbindung mit einem Nystagmus, welcher gemäss des Ersten Ewald'schen Gesetzes überwiegend in der Ebene des betroffenen Bogenganges schlägt ( Abb. 1; $\bullet$ Tab. 1). So kann a

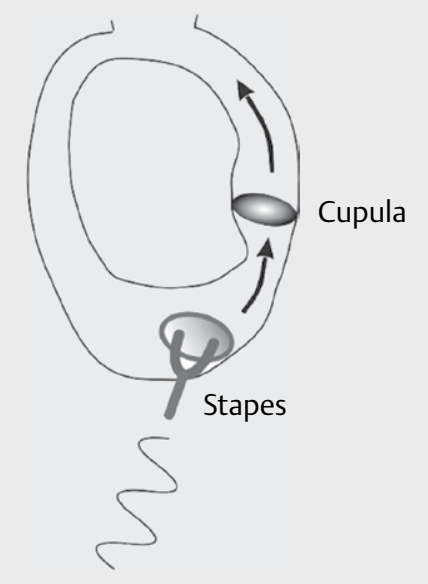

b
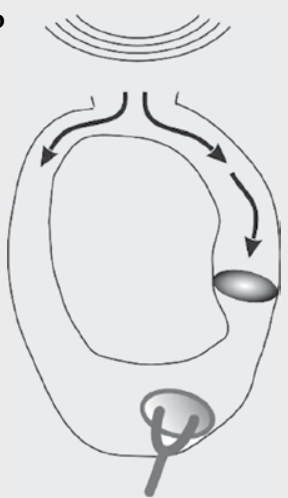

Abb. 4 Endolymphströmung bei Dehiszenz des superioren Bogenganges (SCD). a Druckerhöhung im Mittelohr (z.B. Luftleitungsschall, nasaler Valsalva-Versuch, Hennebert-Zeichen): der ampullofugale = exzitatorische Endolymphstrom resultiert in einem exzitatorischen Nystagmus in der Ebene des superioren Bogenganges. b Druckerhöhung in der mittleren Schädelgrube: Endolymphstrom (ampullopetal = inhibitorisch) und Nystagmusrichtung sind entsprechend dem Dritten Ewald'schen Gesetz entgegensetzt zu a. Siehe auch $>$ Abb. 1 und $>$ Tab. 1.

Tab. 1 Provokationsmanöver für Syndrome des dritten Fensters und Nystagmusbefunde am Beispiel einer Dehiszenz des linken superioren (= anterioren) Bogenganges (nach [142]).

\section{Test/Durchführung}

Hennebert-Zeichen [157]: Druck auf den Tragus

Valsalva mit geschlossener Nase: Nase zuhalten und Luft ins Ohr pusten

Valsalva mit geschlossener Glottis: vorbeugen und anspannen ("als ob man etwas Schweres hochhebt»)

Tullio-Zeichen [139]: Reintöne von 125 bis 4000 Hz über Kopfhörer präsentieren [142]

\section{Effekt}

Erhöhung des Mittelohrdrucks ( $\triangleright$ Abb. $4 \mathbf{4 a}$ )

$\rightarrow$ Druckwelle durch den a-BG Richtung Dehiszenz

$\rightarrow$ ampullofugale (= exzitatorische) Endlymphströmung

Erhöhung des Mittelohrdrucks und des intrakraniellen Drucks. Wenn Erhöhung des Mittelohrdrucks überwiegt ( $\bullet \mathbf{A b b}$. $\mathbf{4 a}$ ):

$\rightarrow$ ampullofugale Endolymphströmung durch den a-BG (vgl. HennebertZeichen)

Erhöhung des intrakraniellen Drucks ( $\bullet$ Abb. $\mathbf{4 b}$ )

$\rightarrow$ Druckwelle von der Dehiszenz durch den a-BG in Richtung Utrikulus

$\rightarrow$ ampullopetale ( $=$ inhibitorische) Endolymphströmung

Schallwelle $\rightarrow$ Gehörknöchelchenkette $\rightarrow$ ovales Fenster $\rightarrow$ oberer

Bogengang $\rightarrow$ Dehiszenz ( $\bullet$ Abb. 4 a)

$\rightarrow$ ampullofugale (=exzitatorische) Endolymphströmung

\section{Nystagmus}

Links rotatorisch mit Downbeat-Komponente ( Abb. 1b)

Links rotatorisch mit Downbeat-Komponente

Rechts rotatorisch mit Upbeat-Komponente ( Abb. 1b)

Links rotatorisch mit Downbeat-Komponente

a-BG = anteriorer Bogengang. 
a

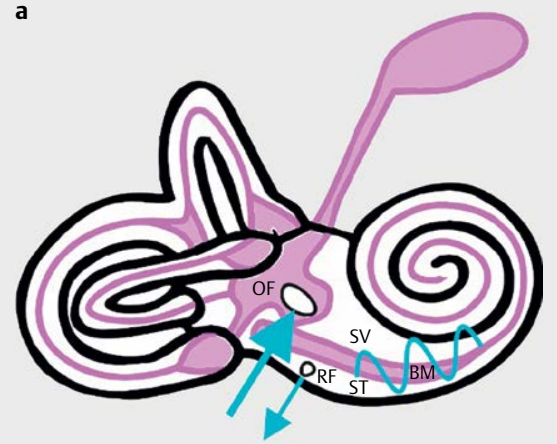

C

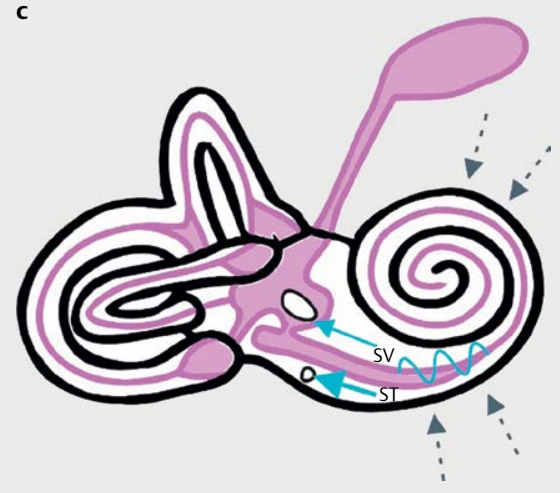

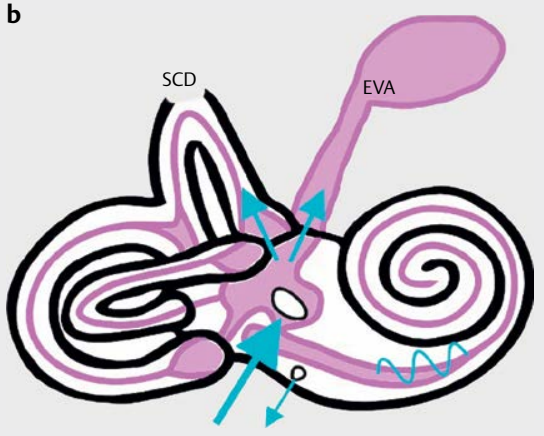

d

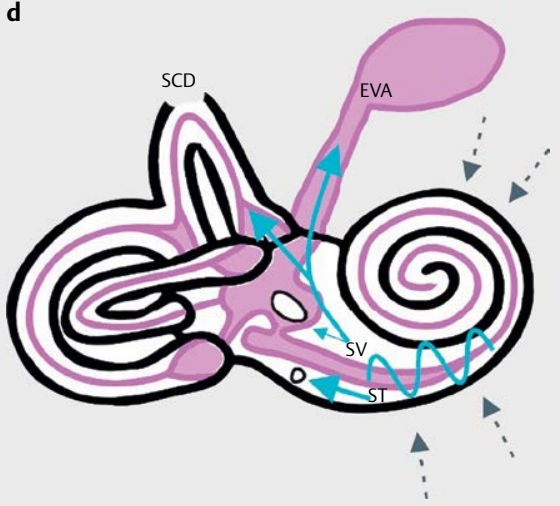

- Abb. 5 Innenohr-bedingte Schallleitungsschwerhörigkeit und knöcherne Hyperakusis bei Syndromen des „dritten Fensters“. a Flüssigkeitsströmung im Innenohr ausgelöst durch Luftleitungs-Schallreize (blauer Pfeil am ovalen Fenster) bei intakter otischer Kapsel. b Im Falle eines dritten Fensters (SCD oder EVA) geht ein Teil der Schallleitungs-Energie über das dritte Fenster verloren, resultierend in einer geringeren Auslenkung der Basilarmembran (BM) und damit einer Verschlechterung der Luftleitungs-Hörschwelle. c Flüssigkeitsströmung im Innenohr ausgelöst durch Knochenleitungs-Schallreize (graue Pfeile) bei intakter otischer Kapsel. $\mathbf{d}$ Im Falle eines dritten Fensters nimmt der Druckgradient zwischen ovalem und rundem Fenster zu, die Auslenkung der Basilarmembran wird verstärkt, und es resultiert eine verbesserte Knochenleitungs-Hörschwelle. Details siehe Kap. 3.2.1 und [131]. Abkürzungen: BM = Basilarmembran, EVA= erweiterter vestibulärer Aquädukt, SCD = Dehiszenz des superioren Bogenganges (superior canal dehiscence), $\mathrm{OF}=$ ovales Fenster, $\mathrm{RF}=$ rundes Fenster, $\mathrm{SV}=\mathrm{Scala}$ vestibuli, $\mathrm{ST}=\mathrm{Scala}$ tympani.

- Tab. 2 Differenzialdiagnostik zwischen Mittelohr- und Innenohrbedingter Schallleitungsschwerhörigkeit [131] (siehe Kapitel 3.2.1).

\begin{tabular}{|c|c|c|}
\hline Test & $\begin{array}{l}\text { Mittelohrbedingte } \\
\text { SLSH }\end{array}$ & $\begin{array}{l}\text { Innenohrbedingte } \\
\text { SLSH }\end{array}$ \\
\hline Tympanometrie & $\begin{array}{l}\text { abgeflacht oder } \\
\text { normal }\end{array}$ & normal \\
\hline Stapediusreflexe & häufig ausgefallen & vorhanden \\
\hline $\begin{array}{l}\text { Otoakustische } \\
\text { Emissionen }\end{array}$ & nicht vorhanden & vorhanden \\
\hline $\begin{array}{l}\text { Knochenleitungs- } \\
\text { schwellen }\end{array}$ & selten $<0 \mathrm{~dB} \mathrm{nHL}$ & $\begin{array}{l}\text { häufig }<0 \mathrm{~dB} \mathrm{nHL} \text { für } \\
\text { Frequenzen }<2 \mathrm{kHz}\end{array}$ \\
\hline ACS VEMPS & $\begin{array}{l}\text { reduziert/ausgefallen } \\
\text { (bereits ab einer SLSH } \\
\text { von } 10 \mathrm{~dB} \text { ) }\end{array}$ & $\begin{array}{l}\text { erhöhte Amplituden, } \\
\text { erniedrigte Schwelle } \\
\text { trotz SLSH }\end{array}$ \\
\hline
\end{tabular}

ACS VEMPs = mit Luftleitungsreizen ausgelöste vestibulär evozierte myogene Potenziale. $\mathrm{nHL}=$ normal hearing level, $\mathrm{SLSH}=$ Schallleitungsschwerhörigkeit. anhand der Nystagmusrichtung der betroffene Bogengang identifiziert werden (Details siehe Kapitel 3.2.2 bis 3.2.5). Lärminduzierter Schwindel (Tullio-Phänomen) [139,140] Ähnlich verhält es sich bei der Übertragung von Schallenergie über Luftleitung auf das Innenohr. Ein Teil der Schallenergie wählt - im wahrsten Sinne des Wortes - den „Weg des geringsten Widerstandes“, also der verbesserten Compliance, zum neu geschaffenen dritten Fenster. Führt die Endolymphströmung wie im Beispiel einer Bogengangsdehiszenz an den vestibulären Rezeptorzellen des Gleichgewichtsorgans vorbei, erzeugt dies kurzfristigen Schwindel und einen korrespondierenden Nystagmus ( $\triangleright$ Abb. 4a; $\triangleright$ Tab. 1). Innenohr-bedingte Schallleitungsschwerhörigkeit Derjenige Teil der Luftleitungs-Schallenergie, der über das dritte Fenster das Innenohr verlässt, gelangt nicht bis zum runden Fenster. Dadurch nimmt der Druckgradient entlang der Basilarmembran der Cochlea vom ovalen zum runden Fenster ab, die Stereozilien der cochleären Haarzellen werden in der Folge weniger stark ausgelenkt, und es resultiert eine Innenohr-bedingte Schallleitungsschwerhörigkeit ( $\mathbf{A} \mathbf{b b}$. 5a, b). Aufgrund der hydrodynamischen Eigenschaften der Innenohrflüssigkeiten betrifft diese überwiegend die tiefen 
Frequenzen < 2 kHz [131]. \ Tab. 2 fasst Unterscheidungsmerkmale zwischen Mittel- und Innenohr-bedingter Schallleitungsschwerhörigkeit zusammen.

Verbesserte Knochenleitung/Autophonie Liegt das dritte Fenster im Bereich des Vestibularorgans oder auf der Seite der cochleären Scala vestibuli, wird ein verbessertes Knochenleitungshören mit Schwellen bis zu $<0 \mathrm{~dB} \mathrm{nHL}$ (normal hearing level) beobachtet («bony hyperacusis»). Bei intakter otischer Kapsel breitet sich Schallenergie, welche über Knochenleitung auf das Innenohr übertragen wird, sowohl in Richtung zum ovalen als auch zum runden Fenster aus ( $\mathbf{A} \mathbf{A b b} . \mathbf{5 c}$ ). Da der Widerstand durch die angrenzende Stapesfussplatte am ovalen Fenster grösser ist als am runden, resultiert ein Druckgradient entlang der Basilarmembran zum runden Fenster. Fliesst ein Teil der Knochenleitungs-Schallenergie über ein drittes Fenster auf Seite der Scala vestibuli ab, reduziert sich der Schalldruck am ovalen Fenster noch weiter, wodurch der Druckgradient zwischen ovalem und rundem Fenster (d. h. über der Basilarmembran) zunimmt ( $\triangleright$ Abb. 5d). Entsprechend kommt es nun zu einer verstärkten Auslenkung der Basilarmembran und damit zu einem verbesserten Knochenleitungshören [131].

Die Patienten berichten typischerweise über eine Autophonie (verstärkte Wahrnehmung von körpereigenen Geräuschen im betroffenen Ohr, z. B. Augenbewegungen, Herzschlag, Kaubewegungen, Darmgeräusche), eine verzerrte Wahrnehmung der eigenen Stimme (Diplakusis) aufgrund der Diskrepanz zwischen Luft- und Knochenleitungshören, Geräuschwahrnehmung der eigenen Schritte oder einen pulssynchronen Tinnitus (Weiterleitung von Flüssigkeitsturbulenzen in Gefässen an die Cochlea über die verbesserte Knochenleitung) [141, 142].

Die Ausprägung dieser vier Symptomkomplexe variiert stark zwischen den einzelnen Krankheitsbildern und von Patient zu Patient. Als Faustregel sollte immer an ein Syndrom des dritten Fensters gedacht werden, wenn zumindest ein Symptom aus einer der o.g. vier Kategorien vorliegt.

\section{TIPP FÜR DIE PRAXIS}

Geben Sie bei Verdacht auf ein Syndrom des dritten Fensters in der Audiometrie-Anforderung an, dass die Knochenleitungsschwellen bis zu Werten $<0 \mathrm{~dB} \mathrm{nHL}$ verfolgt werden $[131,142]$.

\subsubsection{Dehiszenz des superioren Bogenganges}

\subsubsection{Epidemiologie und Ursachen}

Die genaue Inzidenz und Prävalenz der erstmals von Lloyd Minor und Kollegen beschriebenen Dehiszenz des oberen Bogenganges (superior canal dehiscence, SCD) [143] ( $>$ Abb. 6a) in der Gesamtbevölkerung ist nicht genau bekannt, dies hat mehrere Gründe. Zum einen besteht im CT Schläfenbein aufgrund von Volumenmittlungseffekten die Gefahr einer Überdiagnose - sowohl hinsichtlich der Existenz als auch der Grösse der SCD (siehe Kapitel 3.2.2.3) [144, 145]. Zum anderen wird nicht jede SCD symptomatisch [146]. So zeigte sich in einer Studie an konsekutiven Schläfenbein-CTs $(0,625 \mathrm{~mm}$ Schichtdicke) einer Notaufnahme in $5,8 \%$ der untersuchten Schläfenbeine radiologisch eine knöcherne Dehiszenz des oberen Bogenganges [147]. Allerdings wiesen nur 11,8\% dieser Personen die pathognomonischen Symptome oder Untersuchungsbefunde für eine SCD auf. Bezogen auf das Gesamtkollektiv waren dies 0,5\%, was der Prävalenz einer dehiszenten knöchernen Bedeckung des oberen Bogenganges in post mortem Schläfenbeinstudien entspricht [148].

Eine SCD kann spontan auftreten, in ca. einem Viertel der Fälle geht jedoch ein auslösendes Trauma voran, z. B. ein Schädel-HirnTrauma, Pressen im Rahmen einer Geburt [149] oder starkes Husten bei einer Erkältung (siehe hierzu die Fallbeispiele in $>$ Abb. $\mathbf{6}$ und [132]). Es können auch weitere Dehiszenzen, z. B. am Tegmen tympani oder am posterioren Bogengang vorhanden sein (Kapitel 3.2.4) [150-152]. Im Extremfall spricht man von einem «honeycomb
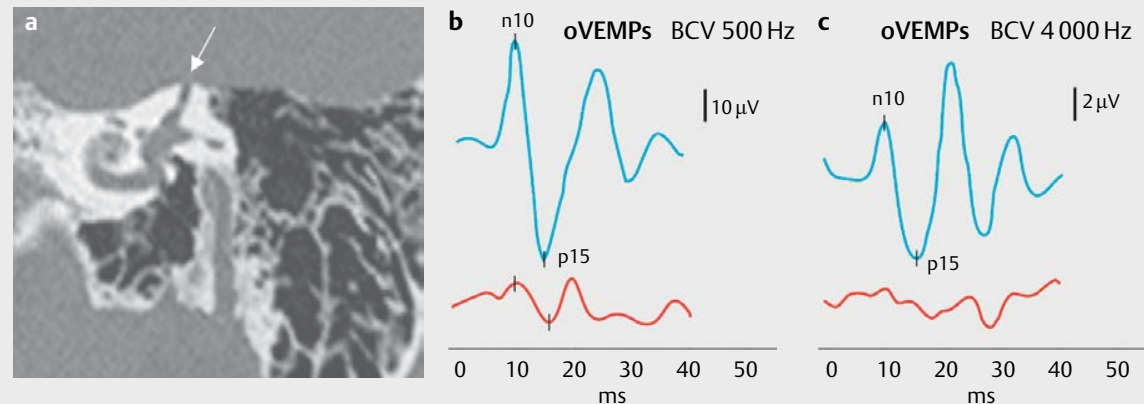

\footnotetext{
- Abb. 6 Dehiszenz des superioren Bogenganges (SCD) links. Die 55-jährige Patientin bemerkte erstmals nach einem viralen Infekt mit starkem Husten ein „Wattegefühl“ im linken Ohr mit Autophonie (Hören des Pulsschlags und der eigenen Schritte im linken Ohr), Diplakusis und einen kurzfristigen Schwankschwindel insbesondere beim Niesen, Husten und Hochheben schwerer Lasten sowie bei lauten Tönen (z. B. Chorgesang). Im Weber-Versuch lateralisierte sie nach links, auch beim Aufsetzen der Stimmgabel am Malleolus medialis links. a Darstellung der knöchernen Dehiszenz zwischen superiorem Bogengang links und mittlerer Schädelgrube (Pfeil) in der koronaren Schichtung des HRCT Schläfenbein. b oVEMPs bei $500 \mathrm{~Hz}$ Knochenleitung (bone conducted vibration, BCV) an Fz (Mitte der Stirn am Haaransatz): deutlich erhöhte n10p15-Amplitude von $67 \mu \mathrm{V}$ für den linken Utrikulus (blau) bei normwertiger Amplitude des rechten Utrikulus (rot, $15 \mu \mathrm{V}$ ), Asymmetrie-Ratio von 60,5\% zu Ungunsten rechts. c oVEMPs bei $4000 \mathrm{~Hz}$ BCV an Fz: für den linken Utrikulus (blau) kann eine n10p15-Amplitude von $9 \mu \mathrm{V}$ ausgelöst werden (Hinweis für SCD), für den rechten Utrikulus nicht (Normalbefund). Hier ist der unterschiedliche Massstab in Vergleich zu b zu beachten. Details siehe Kapitel 3.2.2.
} 
- Tab. 3 Assoziierte Befunde und zugrundeliegende Erkrankungen bei Bogengangs-Dehiszenz-Syndromen.

\section{Superiorer Bogengang}

- Meningeom [371]

- Arrosion durch den Sinus petrosus sup. [372-376]

- Ehlers-Danlos-Syndrom [377]

Posteriorer Bogengang

- Anomalien des Bulbus venae jugularis (hochstehender Bulbus, Divertikel), auch in Assoziation mit einem dehiszenten Aqueductus vestibuli [183-188]

- Fibröse Dysplasie [183]

- latrogen, z.B. nach Mastoidektomie oder VestibularisschwannomChirurgie [183]

- Apex-Cholesteatom [378]

- Kongenitales Cholesteatom des Mastoids [379]

- Eosinophiles Granulom [189]

- Multiple Dehiszenzen des Innenohres [151, 152, 183, 380-381]

- Innenohrfehlbildungen, z.B. erweiterter Aqueductus vestibuli, Mondini-Malformation [185]

- Komplexe Fehlbildungssyndrome, z.B. Apert-Syndrom [185], Hallermann-Streiff-Syndrom (okulo-mandibulo-faziales Syndrom) [382]

\section{Lateraler Bogengang $[142,182]$}

- Cholesteatom

- latrogen

mastoid» $[153,154]$. In seltenen Fällen kann eine SCD auch dadurch erzeugt werden, dass angrenzende Strukturen, wie der Sinus petrosus superior oder ein Meningeom, die knöcherne Begrenzung des oberen Bogenganges zur mittleren Schädelgrube hin arrodieren (Übersicht in > Tab. 3) [155].

\subsubsection{Symptome und Untersuchungsbefunde}

Hinsichtlich der Symptomatik ist die SCD ein wahres "Chamäleon»: Patienten berichten häufig über druck- und lärminduzierten Schwindel (37,4 bzw. 42,7\%), über Autophonie (42,5\%) und einen pulssynchronen Tinnitus (13,7\%) [156]. Bei etwa der Hälfte der Patienten, die sich präoperativ zum Verschluss der Dehiszenz (s. Kapitel 3.2.2.4) vorstellen, kann durch Druck auf den Tragus ein Nystagmus ausgelöst werden (Hennebert-Zeichen) [157]. Die meisten Patienten zeigen einen Nystagmus bei Valsalva-Manöver und/oder bei Präsentation von Reintönen (125-4000 Hz, $110 \mathrm{~dB}$ nHL) auf dem betroffenen Ohr über Kopfhörer [142]. Die jeweiligen Nystagmusrichtungen gemäss der Ewald'schen Gesetze sind in > Tab. 1 zusammengefasst. In seltenen Fällen lässt sich auch ein pulssynchroner Nystagmus - ausgelöst durch pulsierende intrakranielle Druckschwankungen - beobachten [158].

Schliesslich kann eine SCD auch einen BPLS des anterioren Bogenganges (a-BPLS) imitieren (Kapitel 3.3.2, > Tab. 4) [159]: während im Sitzen die knöcherne Dehiszenz im Dach des superioren Bogenganges durch das Gehirn abgedeckt wird, kann es beim Hinlegen zu einem «Unplugging» des Kanals mit ampullofugalem ( = exzitatorischen) Endolymphfluss kommen, welcher - wie beim a-BPLS
- zu einem exzitatorischen Nystagmus des anterioren Bogenganges führt ( $\triangleright$ Abb. 1b).

Gemäss dem ersten Ewald'schen Gesetz korrespondiert die Nystagmusrichtung bei der SCD i.d.R. mit der Ebene des betroffenen superioren Bogenganges [160]. Sollte die Diskrepanz zwischen Nystagmusrichtung und Ebene des superioren Bogenganges sehr gross sein, ist zu überlegen, ob zusätzlich noch eine weitere knöcherne Dehiszenz im posterioren oder horizontalen Bogengang vorliegt (Kapitel 3.2.4 und 3.2.5) [161].

Ein weiterer sensitiver - jedoch in der klinischen Praxis häufig vernachlässigter - Test, ist die Prüfung eines Vibrationsnystagmus (VIN), welcher bei 84-100\% der Patienten mit SCD beschrieben wurde. Bei $100 \mathrm{~Hz}$ Vibrationsfrequenz schlägt dieser überwiegend horizontal in Richtung des betroffenen Ohres als Ausdruck der gesteigerten globalen Sensitivität des dehiszenten Labyrinthes für Vibrationsreize. Bei $500 \mathrm{~Hz}$ zeigt sich überwiegend ein exzitatorischer Nystagmus in der Ebene des betroffenen oberen Bogenganges (Details und neurophysiologische Grundlagen bei [162-164]).

Die knöcherne Hyperakusis auf Seiten der Dehiszenz demarkiert sich im Weber-Test $(512 \mathrm{~Hz})$ mit einer Lateralisation auf die betroffene Seite, häufig sogar dann, wenn die Stimmgabel am medialen Malleolus aufgesetzt wird [165]. Manchmal genügt es bereits, den Patienten summen zu lassen, um einen Nystagmus zu provozieren $[141,166]$. Im Reintonaudiogramm zeigt sich der oben beschriebene typische Befund eines dritten Fensters mit einer tieffrequenten Innenohr-bedingten Schallleitungsschwerhörigkeit und Knochenleitungsschwellen $<0 \mathrm{~dB} \mathrm{nHL}$.

Während früher häufig erniedrigte Schwellen für die Auslösung von cVEMPs bei $500 \mathrm{~Hz}$ Luftleitungsreiz auf der betroffenen Seite als Indiz für eine SCD gewertet wurden, werden heute wegen der grösseren diagnostischen Genauigkeit meist Amplitudenunterschiede der oVEMPs als diagnostischer Marker herangezogen [167]. Insbesondere ist eine unter dem kontralateralen Auge gemessene, im Seitenvergleich erhöhte oVEMP n10p15-Amplitude bei $500 \mathrm{~Hz}$ Luft- oder Knochenleitungsstimulus ein zuverlässiger Indikator für eine SCD mit einer Sensitivität und Spezifität $>90 \%$ ( $\triangleright$ Abb. $6 \mathbf{b}$ ). Die genauen Werte hängen von den gewählten Stimulusparametern, Kontrollgruppen und Cut-off-Werten der jeweiligen Studien ab (Details siehe u. a. [167-170]). Die diagnostische Genauigkeit lässt sich weiter steigern durch die Messung der oVEMPs bei $4 \mathrm{kHz}$ $[171,172]$. Während sich für das Innenohr mit intakter otischer Kapsel bei diesen Frequenzen normalerweise keine oVEMPs mehr auslösen lassen, spricht der Nachweis von oVEMP-Antworten bei 4 kHz mit einer diagnostischen Genauigkeit von ca. $90 \%$ für eine SCD ( Abb. 6c; siehe $[138,173]$ für die neurophysiologischen Grundlagen).

Obwohl erhöhte VEMP-Amplituden pathognomonisch für ein Syndrom des dritten Fensters sind, finden sich diese auch in geringerem Ausmass bei anderen Erkrankungen, welche die Flüssigkeitsmechanik im Innenohr beeinflussen, z. B. bei M. Menière im Anfangsstadium [12] und bei intracochleären Schwannomen [174].

Schliesslich lassen sich in der Elektrocochleografie (ECochG) bei Patienten mit SCD - ähnlich wie bei Endolymphhydrops bzw. M. Menière - erhöhte Werte für den Quotienten aus Summationspotential (SP) und Aktionspotential (AP) messen. Eine mögliche Erklärung hierfür ist die Abnahme des Perilymphdruckes bedingt durch die knöcherne Dehiszenz, was - relativ gesehen - zu einer 
> Tab. 4 Wichtige seltene Differenzialdiagnosen des benignen paroxysmalen Lagerungsschwindels (BPLS, Details in Kapitel 3.3.2).

\begin{tabular}{|c|c|c|c|}
\hline Krankheitsbild & Klinische Befunde & Pathophysiologie & „red flags“ \\
\hline Vestibularisschwannom & \multirow[t]{2}{*}{$\begin{array}{l}\text { Richtungswechselnder } \\
\text { positionsabhängiger } \\
\text { Nystagmus }\end{array}$} & $\begin{array}{l}\text { Unterschiedlicher Druck des Tumors auf } \\
\text { die Fasern des N. vestibularis abhängig } \\
\text { von der Körperposition [383] }\end{array}$ & \multirow[t]{2}{*}{$\begin{array}{l}\text { Einseitige cochleovestibuläre } \\
\text { Unterfunktion }\end{array}$} \\
\hline $\begin{array}{l}\text { Intralabyrinthäres } \\
\text { Schwannom (Kap. 3.4.1) }\end{array}$ & & $\begin{array}{l}\text { Unterschiedlicher Einfluss der Tumormasse } \\
\text { auf den Endolymphfluss abhängig von der } \\
\text { Körperposition [384] }\end{array}$ & \\
\hline $\begin{array}{l}\text { Dehiszenz des superioren } \\
\text { Bogenganges (Kap. 3.2.2) }\end{array}$ & $\begin{array}{l}\text { Nystagmus in der Ebene des } \\
\text { anterioren Bogenganges } \\
\text { (a-BG) beim Abliegen }\end{array}$ & $\begin{array}{l}\text { „Unplugging“ des a-BG beim Abliegen } \\
\text { [159] }\end{array}$ & $\begin{array}{l}\text { Keine Nystagmusumkehr beim } \\
\text { Aufsitzen }\end{array}$ \\
\hline $\begin{array}{l}\text { Erweiterter vestibulärer } \\
\text { Aquädukt (Kap. 3.2.6) }\end{array}$ & $\begin{array}{l}\text { Nystagmus bei schnellen } \\
\text { Kopfbewegungen und } \\
\text { Positionswechseln }\end{array}$ & $\begin{array}{l}\text { Ungedämpfte Übertragung intrakranieller } \\
\text { Druckschwankungen auf die Endolymph- } \\
\text { räume [207] }\end{array}$ & $\begin{array}{l}\text { Keine Latenz, keine Nystagmusumkehr } \\
\text { bei Positionswechsel, Hörminderung }\end{array}$ \\
\hline $\begin{array}{l}\text { Labyrinthischämie (Kap. } \\
\text { 2.2.1) }\end{array}$ & $\mathrm{p}$-BPLS & $\begin{array}{l}\text { Ischämie im posterioren Bogengang und } \\
\text { der Cochlea bei Infarkt der A. cochlearis } \\
\text { communis [72] }\end{array}$ & $\begin{array}{l}\text { Simultane akute ipsilaterale sensori- } \\
\text { neurale Schwerhörigkeit }\end{array}$ \\
\hline Kleinhirnläsionen & $\begin{array}{l}\text { Zentraler Lage- oder } \\
\text { Lagerungsnystagmus }\end{array}$ & $\begin{array}{l}\text { Mittelliniennahe Läsion des Cerebellums } \\
\text { (Vermis, Nodulus, Pedunculus cerebelli } \\
\text { sup.) [50] }\end{array}$ & $\begin{array}{l}\text { siehe Übersicht in Kap. } 3.3 .2 \text { (bis auf } \\
\text { Hörminderung) }\end{array}$ \\
\hline
\end{tabular}

Erhöhung des Endolymphdruckes führen kann (sog. «Endolymphhydrops e vacuo»). Die ECochG lässt sich auch zum intraoperativen Monitoring einsetzen. Nach erfolgreichem Verschluss einer SCD normalisieren sich die auffälligen neurophysiologischen Befunde, wie der SP/AP-Quotient $[175,176]$ und die VEMPs [12].

\subsubsection{Bildgebung}

Als Goldstandard gilt hier das hochauflösende Schläfenbein-CT (high resolution computed tomography, HRCT) mit Schichtdicken von $\leq 0,625$ mm und Rekonstruktion in der Pöschl-Ebene (senkrecht zur Längsachse des Felsenbeins, d. h. in der Ebene des oberen Bogenganges) und der Stenvers-Ebene (senkrecht zur Pöschl-Ebene) [130]. Inzwischen wird die digitale Volumentomographie (DVT)/Cone beam Tomografie (CBT) als mindestens gleichwertig zum HRCT in der SCD-Diagnostik angesehen: die Strahlendosis ist im Vergleich zum HRCT geringer, die Auflösung besser und die Kosten niedriger $[177,178]$.

Stark T2-gewichtete MRT-Aufnahmen (z. B. CISS oder FIESTA) detektieren eine Dehiszenz des oberen Bogenganges zwar mit der gleichen Sensitivität wie ein hochauflösendes CT, allerdings wird in $40 \%$ der Fälle eine falsch-positive Diagnose im Vergleich mit dem CT gestellt. Daher sollte bei Verdacht auf eine SCD im SchläfenbeinMRT ein HRCT oder DVT zur Sicherung der Diagnose durchgeführt werden [179].

Aufgrund der generellen Überschätzung von Dehiszenzen des oberen Bogenganges in der Bildgebung wird in den von Ward et al. [161] vorgeschlagenen diagnostischen Kriterien neben dem radiologischen Nachweis einer Dehiszenz im HRCT auch das Vorhandensein von mindestens einem pathognomonischen Symptom (d. h. lärm- oder druckinduzierter Schwindel, Autophonie, pulssynchroner Tinnitus) und mindestens einem audiovestibulären Befund gefordert, der durch das dritte Fenster erklärt werden kann (d. h. negative Knochenleitungshörschwelle, charakteristischer VEMP- oder ECochG-Befund).

\subsubsection{Therapie}

Häufig wirkt allein die Information über die Existenz der Dehiszenz und die Pathophysiologie des Krankheitsbildes entlastend für die Patienten, insbesondere die Tatsache, dass es für die teilweise bizarr anmutenden Symptome wie das Hören der eigenen Augenund Darmbewegungen eine schlüssige Erklärung gibt. In vielen Fällen können Trigger wie Lärm oder starke Druckänderungen vermieden werden [142]. Werden die Symptome hauptsächlich durch Druckänderungen im Bereich des Mittelohres ausgelöst (z. B. bei schnellen Höhenwechseln), kann die Anlage eines Paukenröhrchens auf der betroffenen Seite hilfreich sein [177].

Der operative Verschluss der knöchernen Dehiszenz stellt die einzige kausale Therapie dar, wofür sich ca. 30-50\% der Patienten entscheiden. Die unterschiedlichen Zugangswege (transtemporal vs. transmastoidal) und Verschlusstechniken («Plugging», «Resurfacing» oder die Kombination aus beidem) mit ihren Indikationen, Vor- und Nachteilen sowie Risiken und Erfolgsraten werden ausführlich bei $[142,161,177]$ dargestellt. Im Falle eines «honeycomb mastoid» mit multiplen Dehiszenzen werden inzwischen auch mittels "computer-aided design» (CAD) massgeschneiderte Glaskeramikplatten für die Wiederherstellung der Mastoidoberfläche verwendet [154].

\subsection{3 "Near dehiscence"-Syndrom des superioren Bogenganges}

Auch eine nicht komplett dehiszente, sehr dünne und nachgiebige knöcherne Bedeckung des oberen Bogenganges (Dicke $<0,1 \mathrm{~mm}$ ) kann zu einem Locus minoris resistentiae mit den typischen Symptomen, klinischen Zeichen und elektrophysiologischen Untersuchungsbefunden einer SCD führen [180]. In der Studie von Carey et al. [148] wurde bei 1,4\% der Schläfenbeinpräparate ein derartig dünner Knochen gesehen. Im Vergleich zur kompletten Dehiszenz sind die klinischen Befunde bei einer «Near dehiscence» des oberen Bogenganges meist geringer ausgeprägt $[180,181]$. Die chirurgi- 
sche Therapie besteht entweder in einer Verstärkung der knöchernen Bedeckung des oberen Bogenganges (z. B. mit Faszie oder Knochenzement) oder in einer Kombination aus «Plugging» und anschliessendem «Resurfacing». In einigen Fällen entpuppt sich eine präoperativ im CT diagnostizierte Dehiszenz intraoperativ als «Near dehiscence» - ein weiteres anschauliches Beispiel für die Überdiagnose einer SCD in der Bildgebung $[146,180]$.

\section{TIPP FÜR DIE PRAXIS}

Die Entscheidung für eine operative Therapie sollte sowohl für die Dehiszenz als auch die "Near dehiscence» des superioren Bogenganges immer von der klinischen Präsentation und dem Vorhandensein pathognomonischer audiovestibulärer Befunde abhängig gemacht werden - nie alleine von der Bildgebung.

\subsubsection{Dehsizenz des posterioren Bogenganges}

Diese ist mit einer Prävalenz von 0,2\% in einer post mortem Schläfenbeinstudie seltener als die SCD [95]. Die Dehiszenz des posterioren Bogenganges (posterior canal dehiscence, PCD) findet sich häufig in Assoziation mit einem hochstehenden Bulbus venae jugularis (30\%), iatrogen bedingt (15\%) oder bei einer fibrösen Dysplasie des Schläfenbeins ( $\triangleright$ Tab. 3) $[182,183]$. Ein erweiterter oder hochstehender Bulbus venae jugularis kann aufgrund der anatomischen Nachbarschaft auch zu einer Dehsizenz des Aqueductus vestibuli führen, welche ebenfalls als drittes Fenster wirken kann [184-188].

Die Symptome und klinischen Zeichen einer Dehiszenz des posterioren Bogenganges entsprechen denen der SCD. Allerdings schlägt der Nystagmus nun in der Ebene des betroffenen posterioren Bogenganges, d. h. im Falle einer Exzitation rotatorisch zur betroffenen Seite mit Upbeat-Komponente - analog zum p-BPLS ( $\triangleright$ Abb. 1a) $[48,189,190]$. Auch Patienten mit PCD zeigen häufig die für Syndrome des dritten Fensters charakteristische Innenohrbedingte Schallleitungsschwerhörigkeit mit negativen Knochenleitungsschwellen für Frequenzen $<2 \mathrm{kHz}$ im Tonaudiogramm [191]. Aufgrund der Seltenheit dieses Krankheitsbildes liegen noch keine systematischen Untersuchungen zu den entsprechenden cund oVEMP-Antworten vor. Es gibt jedoch Fallberichte mit ipsilateral erniedrigten Schwellen und erhöhten Amplituden für die cVEMPs $[183,185]$.

Die radiologische Diagnostik wird analog zur SCD durchgeführt, inklusive Rekonstruktion in der Pöschl- und Stenvers-Ebene. Die chirurgische Therapie erfolgt durch ein transmastoidales Plugging des posterioren Bogenganges. Im Falle eines hochstehenden oder dilatierten Bulbus venae jugularis wird zusätzlich zum Plugging die natürliche Grenze zwischen Bulbus und Bogengang verstärkt, z. B. durch die Interposition von Knorpel und Faszie [192-194].

\subsubsection{Dehiszenz des horizontalen Bogenganges}

Im Vergleich zur SCD und PCD ist ein drittes Fenster im horizontalen Bogengang ein echter «Kolibri». Als mögliche Ursache für die Seltenheit von Dehiszenzen an dieser Stelle wird vermutet, dass der horizontale Bogengang - im Gegensatz zum superioren und posterioren Kanal - nicht direkt an das Schädelinnere angrenzt. Da- durch ist der Knochen des knöchernen Labyrinths an dieser Stelle nicht den pulsierenden intrakraniellen Druckschwankungen ausgesetzt, die als ein möglicher ätiopathogenetischer Faktor bei der Entstehung von Bogengangsdehiszenzen diskutiert werden [182]. Meist findet sich eine Dehiszenz des horizontalen Bogenganges bei Cholesteatomen des Mittelohrs oder als Folge von operativen Eingriffen ( $\triangleright$ Tab. 3) [142].

Der druck- und lärminduzierte Nystagmus schlägt hier gemäss dem ersten Ewald'schen Gesetz rein horizontal [195]. Falls die Dehiszenz des lateralen Bogenganges im Rahmen einer Mittelohrerkrankung entstanden ist, ist bei der audiovestibulären Diagnostik darauf zu achten, dass die klinischen Zeichen des dritten Fensters von denen einer Mittelohrpathologie maskiert werden können (> Tab. 2).

Während die bisher behandelten Erkrankungen durch eine nicht-natürlich vorhandene knöcherne Dehiszenz bedingt sind, können auch abnorme Erweiterungen von natürlichen neurovaskulären Foramina zu den typischen Syndromen eines dritten Fensters führen Hierzu gehören insbesondere der erweiterte Aqueductus vestibuli (Kapitel 3.2.6) und die X-chromosomale familiäre Schwerhörigkeit mit Stapes-Gusher (Kapitel 3.2.7).

\subsubsection{Erweiterter Aqueductus vestibuli/Saccus endolympha- ticus}

Der häufigste Vertreter der abnorm erweiterten natürlichen Foramina ist der erweiterte Aqueductus vestibuli (large vestibular aqueduct, LVA oder enlarged vestibular aqueduct, EVA), welcher meist auch mit einem erweiterten Saccus endolymphaticus einhergeht ( $>$ Abb. 7). Der erweiterte vestibuläre Aquädukt ist in 60-80\% der Fälle bilateral vorhanden und häufig mit anderen Fehlbildungen des Innenohres verknüpft [196]. Er ist die häufigste Innenohrfehlbildung bei Kindern mit angeborener Schwerhörigkeit (0,6-13\%) [197].

\subsubsection{Radiologische Diagnostik}

Nach den sog. Cincinatti-Kriterien liegt ein EVA vor, wenn der knöcherne Aqueductus vestibuli im axialen HRCT des Schläfenbeins an der Apertura operculi einen Durchmesser von > 1,9 mm hat bzw. in der Mitte zwischen Vestibulum und Operculum einen Durchmesser von>0,9 mm (siehe hierzu das Fallbeispiel in > Abb. 7). Als Faustregel gilt, dass der Durchmesser des Aquädukts nicht grösser als der des benachbarten posterioren Bogenganges sein sollte [198]. HRCT und Schläfenbein-MRT besitzen die gleiche diagnostische Wertigkeit und werden komplementär verwendet. Die Darstellung des Saccus endolymphaticus in der T2- oder CISS-Sequenz des MRT gilt bereits als sicheres Indiz für einen erweiterten vestibulären Aquädukt, da ersterer normalerweise nicht im MRT sichtbar ist $[197,199]$.

\subsubsection{Pathophysiologie}

Der erweiterte vestibuläre Aquädukt ist ein wahres Chamäleon der seltenen Innenohrerkrankungen. Um die mannigfaltigen audiovestibulären Symptome dieses Krankheitsbildes zu verstehen, lohnt sich ein kurzer Blick auf die zugrundeliegende Pathophysiologie. Häufig liegt beim EVA eine homozygote Mutation des SLC26A4Gens vor (siehe Kapitel 3.2.6.6 und \ Tab. 5) [200, 201]. Das Genprodukt Pendrin ist ein Anionen-Austauscher, welcher im Innenohr 

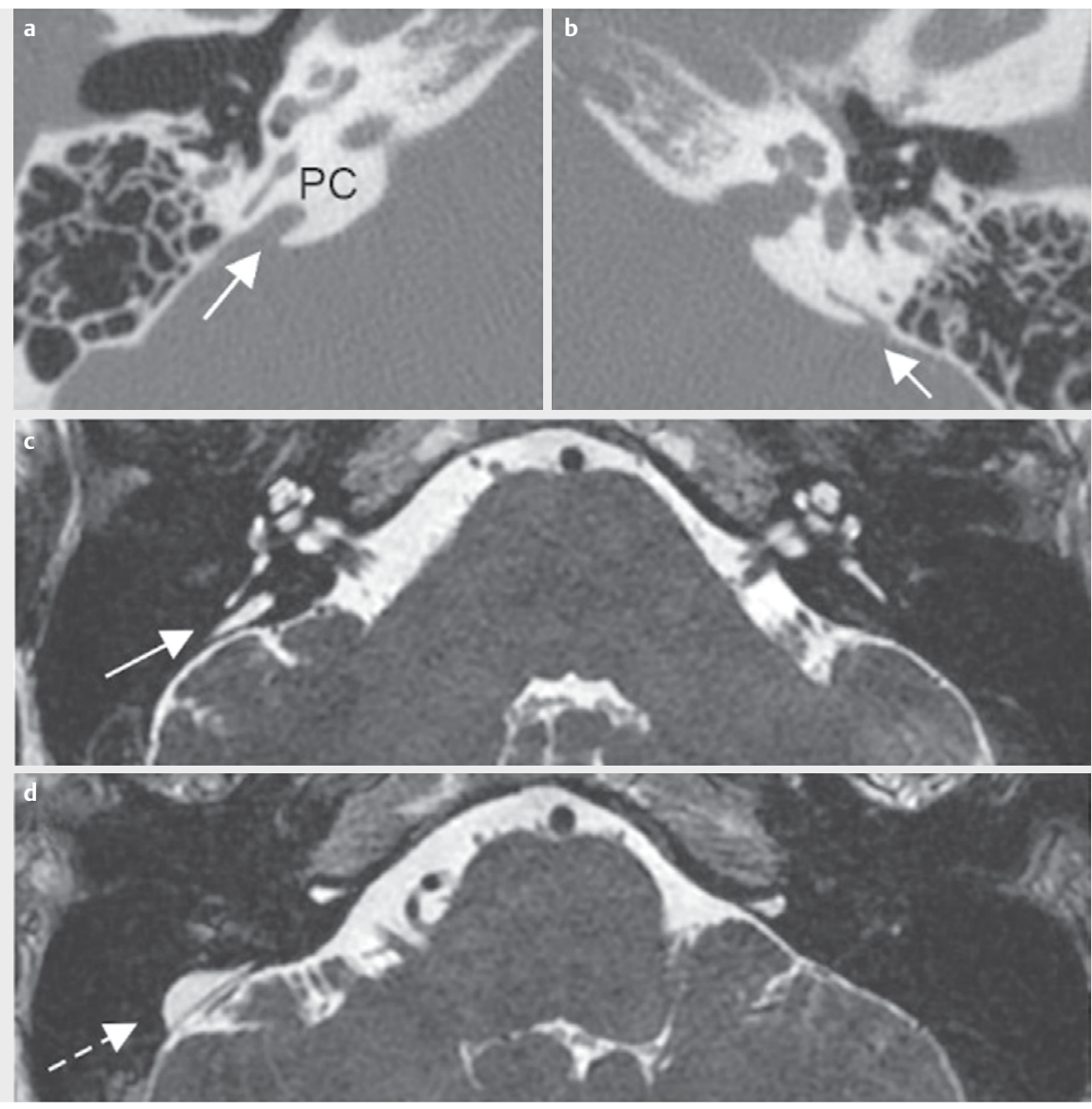

Abb. 7 Erweiterter Aqueductus vestibuli (EVA) rechts. Die 27-jährige Patientin berichtete über kurzfristige Schwankschwindelsensationen beim Niesen, Husten und Pressen, welche erstmals nach Problemen mit dem Druckausgleich bei einem Fallschirmsprung aufgetreten waren. a In den axialen Schichten des HRCT des Schläfenbeins zeigte sich rechts ein EVA (Pfeil, Durchmesser =3,1 mm an der Apertura operculi). Damit ist der Durchmesser des Aquäduktes deutlich grösser als der des benachbarten posterioren Bogenganges (PC). b Normalbefund links. $\mathbf{c}$ und $\mathbf{d}$ In der T2-Wichtung des MRT bestätigte sich die Diagnose eines EVA rechts (Pfeil in c), zudem demarkierte sich ein erweiterter Saccus endolymphaticus rechts (gestrichelter Pfeil in d). Normalbefund links: Ductus und Saccus endolymphaticus nicht im MRT sichtbar.

u. a. in den Epithelien des endolymphatischen Sacks exprimiert wird und $\mathrm{HCO} 3-$ Ionen im Austausch gegen $\mathrm{Cl}^{-}$in den Endolymphraum transportiert, was für die Aufrechterhaltung des $\mathrm{pH}$-Wertes in der Endolymphe von zentraler Bedeutung ist. Aus Untersuchungen an Mausmodellen mit Mutationen des Slc26a4-Gens ist bekannt, dass es aufgrund der defekten Funktion von Pendrin zu einem Absinken des pH-Wertes in der Endolymphe kommt [202, 203]. Die Konsequenzen für die Flüssigkeits- und lonenhomöostase des Innenohres sowie die daraus resultierenden klinischen Korrelate sind in - Tab. 6 zusammengefasst.

\subsubsection{Audiologische Symptome und Befunde}

Die Befunde im Reintonaudiogramm reichen von einer tieftonbetonten Innenohr-bedingten Schallleitungsschwerhörigkeit mit teils negativen Knochenleitungsschwellen («drittes Fenster») bis hin zu einer hochgradigen sensorineuralen Schwerhörigkeit [204-207]. Zusätzlich zu einer langsamen Progression des Hörverlustes existieren auch Fallberichte einer akuten Hörminderung nach leichtem Schädel-Hirn-Trauma oder Druckänderungen im Schädelinneren bzw. Mittelohr (z. B. Valsalva-Manöver, schnelle Höhenwechsel). Teilweise führen diese Ereignisse auch zu einer Erstmanifestation eines bisher stummen EVA (siehe hierzu das Fallbeispiel in > Abb. 7). Basierend auf diesen Erfahrungen wird Patienten häufig empfohlen, Kontaktsportarten oder Aktivitäten mit häufiger Druckänderung (z. B. Tauchen, Fallschirmspringen, Gewichtheben) zu vermeiden. Letztendlich ist aber unklar, ob hier tatsächlich ein kausaler Zusammenhang oder vielmehr ein «reporting bias» besteht [208]. Die langfristige Progression des sensorineuralen Hörverlustes bei EVA scheint unabhängig von Kopftraumata stattzufinden [209].

\subsubsection{Vestibuläre Symptome und Befunde}

Im Vergleich zu den audiologischen Effekten des EVA ist über die vestibulären Auswirkungen bislang nur wenig bekannt. Die vestibulären Symptome reichen von kurzfristigem lärm- oder druckinduziertem Schwindel bis hin einem dauerhaften Unsicherheitsgefühl und spiegeln so - ähnlich wie die audiologischen Befunde - die komplette Bandbreite zwischen drittem Fenster und chronischer cochleovestibulärer Unterfunktion wider [205, 206] (> Tab. 6). Ähnlich wie bei beim Hörverlust wird auch für die vestibulären Symptome in Fallberichten eine Assoziation mit Bagatelltraumata/ Druckänderungen berichtet $[205,210,211]$. 
Drittes Fenster Als charakteristische Zeichen eines dritten Fensters wurden bei Patienten mit EVA ein positives Tullio-Phänomen und ein Vibrationsnystagmus in Richtung des betroffenen Ohres beschrieben [205, 207]. Zudem wurden erniedrigte c- und oVEMPSchwellen sowie erhöhte oVEMP-Amplituden für das entsprechende Labyrinth gemessen [204, 205, 212-214]. Im Gegensatz zum SCD wurde die Erhöhung der oVEMP-Amplituden bislang aber nur für Frequenzen $<2 \mathrm{kHz}$ nachgewiesen, möglicherweise weil beim EVA keine Schall- oder vibrationsinduzierte Stimulation von Bogengangsneuronen stattfindet (siehe Kapitel 3.2.2.2 und [138]).

Die Auslösung von Schwindel und Nystagmus bei schnellen Kopfdrehungen und Positionswechseln wird wahrscheinlich durch die ungedämpfte Übertragung intrakranieller Druckschwankungen auf die Innenohrflüssigkeit im Rahmen des dritten Fensters ausgelöst. Im Gegensatz zum BPLS tritt dieser positionsabhängige Nystagmus ohne Latenz auf, lässt sich nicht einem bestimmten Bogen- gang zuordnen und bessert sich nicht durch Repositionsmanöver [207] ( Tab. 4).

Benigner paroxysmaler Lagerungsschwindel Allerdings findet sich bei Patienten mit EVA auch in ca. $20 \%$ der Fälle ein "echter» BPLS, welcher sich durch Repositionsmanöver therapieren lässt und wahrscheinlich durch die Störung der Calciumhomöostase in der Endolymphe bedingt ist ( Tab. 6). Typisch für den EVA-assoziierten BPLS ist ein rezidivierendes Auftreten mit Beteiligung mehrerer, wechselnder Bogengänge sowie eine Assoziation mit Hörminderung $[211,215,216]$.

Menière-artige Symptomatik Menière-ähnliche Symptome mit Schwindel und Hörminderung über mehrere Stunden wurden bei Patienten mit EVA erstmals von Valvassori et al. 1969 [217] und danach in mehreren klinischen Studien beschrieben [218, 219]. Passend hierzu liess sich bei sechs Patienten mit EVA in beiden Innenohren ein cochleovestibulärer endolymphatischer Hydrops im Schläfenbein-MRT nachweisen [220]. In einer anderen Studie fand

> Tab. 5 Genetisch bedingte Erkrankungen des vestibulären Labyrinths (modifiziert nach [224, 354]).

\begin{tabular}{|c|c|c|c|}
\hline Gen (Genprodukt) & Lokus & Assoziierte Erkrankungen (Erbgang) & Klinisches Bild \\
\hline $\begin{array}{l}\text { VHL (Von Hippel-Lindau tumor } \\
\text { suppressor) }\end{array}$ & $3 p 25.3$ & Von-Hippel-Lindau-Syndrom (AD) & $\begin{array}{l}\text { Multiple Tumoren } \\
\text { + ELST in 3,6\% der Fälle [280] } \\
\text { (Kap. 3.4.2.2) }\end{array}$ \\
\hline$?$ & $6 q$ & Familiäre bilaterale Vestibulopathie (AD) & $\begin{array}{l}\text { BVP [385] } \\
\text { (Kap. 4.1.3.4) }\end{array}$ \\
\hline POU4F3 & $5 q 32$ & DFNA15 (AD) & \multirow{7}{*}{$\begin{array}{l}\text { Progressive sensorineurale Schwerhörigkeit } \\
\text { mit variabler vestibulärer Dysfunktion [354] }\end{array}$} \\
\hline GRHL2 (grainyhead-like 2) & $8 q 22.3$ & DFNA28 (AD) & \\
\hline $\begin{array}{l}\text { CLIC5 (chloride intracellular } \\
\text { channel 5) }\end{array}$ & $6 p 12.3$ & DFNB102/103 (AR) & \\
\hline \multirow[t]{2}{*}{$\mathrm{COCH}$ (Cochlin) } & $14 q 12$ & DFNA9 (AD) & \\
\hline & & DFNB110 (AR) & \\
\hline \multirow[t]{3}{*}{ MYO7A (Myosin 7A) } & \multirow[t]{3}{*}{$11 q 13.5$} & DFNA11 (AD) & \\
\hline & & DFNB2 (AR) & \\
\hline & & Usher-Syndrom 1B (USH1B) (AR) & $\begin{array}{l}\text { Schwerhörigkeit/Taubheit + variable } \\
\text { vestibuläre Funktionsstörung + Retinitis } \\
\text { pigmentosa [358-359] } \\
\text { (Kap. 4.1.3.4) }\end{array}$ \\
\hline \multirow[t]{2}{*}{ SLC26A4 bzw. PDS (Pendrin) } & \multirow[t]{2}{*}{$7 q 22.3$} & DFNB4 mit EVA (AR) & $\begin{array}{l}\text { Erweiterter vestibulärer Aquädukt } \\
\text { + Schwerhörigkeit/Taubheit } \\
\text { + variable vestibuläre Funktionsstörung } \\
\text { (Kap. 3.2.6) }\end{array}$ \\
\hline & & Pendred-Syndrom (AR) & $\begin{array}{l}\text { Zusätzlich: euthyreote (hypothyreote) Struma } \\
\text { [200-202, 225-226] } \\
\text { (Kap. 3.2.6.6) }\end{array}$ \\
\hline POU3F4 & Xq21.1 & $\begin{array}{l}\text { X-chromosomale familiäre Schwerhörigkeit mit } \\
\text { Stapes-Gusher } \\
\text { DFNX2 (XR) }\end{array}$ & $\begin{array}{l}\text { „incomplete partition type III“ [77] } \\
\text { Angeborene Schwerhörigkeit/Taubheit, } \\
\text { „Korkenzieher-Cochlea“, drittes Fenster } \\
\text { zwischen Cochlea und innerem Gehörgang } \\
\text { (siehe Kapitel 3.2.7) }\end{array}$ \\
\hline
\end{tabular}

$A D=$ autosomal-dominant, $A R=$ autosomal-rezessiv, $B V P=$ bilaterale Vestibulopathie, DFNA= autosomal-dominante familiäre nicht-syndromale Schwerhörigkeit, DFNB = autosomal-rezessive familiäre nicht-syndromale Schwerhörigkeit, DFNX2=X-chromosomale familiäre Schwerhörigkeit mit Stapes-Gusher, ELST = Tumor des endolymphatischen Sacks, EVA = erweiterter vestibulärer Aquädukt, USH1B = Usher-Syndrom Typ 1B, VHL = vonHippel-Lindau-Syndrom. 
> Tab. 6 Pathophysiologie des Syndroms des erweiterten Ductus/Saccus endolymphaticus (Kap. 3.2.6).

\begin{tabular}{|c|c|c|}
\hline Pathophysiologie & Konsequenz & Klinisches Korrelat \\
\hline \multirow{2}{*}{$\begin{array}{l}\text { Expression/Funktion epithelialer Natriumkanäle } \\
(\text { ENaCs) im Saccus endolymphaticus } \downarrow \\
{[203,386]} \\
\rightarrow \text { Flüssigkeitsretention im Endolymphraum } \uparrow\end{array}$} & $\begin{array}{l}\text { - Dilatation des Ductus und Saccus } \\
\text { endolymphaticus }\end{array}$ & " « Drittes Fenster » \\
\hline & " Endolymphhydrops [386-387] & - Menière-artige Symptomatik \\
\hline $\begin{array}{l}\text { Funktion epithelialer Kationenkanäle (TRPV 5/6) } \\
\text { im Saccus endolymphaticus } \downarrow[388]\end{array}$ & $\begin{array}{l}{\left[\mathrm{Ca}^{2+}\right] \uparrow \text { in der Endolymphe }} \\
\text { " "Riesen-Otolithen » }\left(\mathrm{CaCO}_{3}\right) \text { im } \\
\text { Utrikulus, Calciumoxalat-Kristalle im } \\
\text { Sakkulus } \\
\text { " Degeneration der Otolithenmembran } \\
\text { [389] }\end{array}$ & Benigner paroxysmaler Lagerungsschwindel \\
\hline Erweiterte Endolymphräume & $\begin{array}{l}\text { Ungedämpfte Übertragung von intrakrani- } \\
\text { ellen Druckschwankungen auf das } \\
\text { cochleo-vestibuläre Sinnesepithel [206] }\end{array}$ & $\begin{array}{l}\text { - Progressive cochleo-vestibuläre Unterfunktion } \\
\text { " Mögliche Assoziation zwischen Bagatelltrauma } \\
\text { und akuter cochleärer/vestibulärer Funktions- } \\
\text { minderung }\end{array}$ \\
\hline
\end{tabular}

sich bei $75 \%$ der EVA-Patienten eine Diskrepanz zwischen reduzierter kalorischer Erregbarkeit und normalem vHIT-Gain für den horizontalen Bogengang - ähnlich wie bei M. Menière [221] - als Indikator für einen Endolymphhydrops [222].

Chronische vestibuläre Unterfunktion Als Zeichen für eine chronische unilaterale oder bilaterale peripher-vestibuläre Unterfunktion demarkieren sich folgende Befunde: eine reduzierte kalorische Erregbarkeit des horizontalen Bogenganges, Einstellsakkaden im Kopf-Impulstest [211, 219,222], ein Vibrationsnystagmus in Richtung des Ohres mit der besseren vestibulären Funktion [205] oder reduzierte VEMP-Amplituden auf der betroffenen Seite [213].

Assoziation mit vestibulärer Migräne Schliesslich wurde eine Assoziation von EVA mit (vestibulärer) Migräne beschrieben $[205,219]$ - ähnlich wie bei der SCD $[142,177]$. Hier ist zu erwägen, dass die vestibuläre Hypersensitivität bedingt durch das zusätzliche dritte Fenster als Trigger für Migränesymptome wirken könnte.

\subsubsection{Therapie}

Die therapeutischen Optionen bei EVA sind äusserst begrenzt. Bei einer klaren Assoziation von Hörminderung und Schwindel mit Triggern wie Lärm und / oder Druckänderungen sollten diese möglichst vermieden werden. Bei akuter Verschlechterung der cochleovestibulären Funktion kommen intratympanale oder systemische Glukokortikoide zum Einsatz [206]. Im Falle einer an Taubheit grenzenden sensorineuralen Schwerhörigkeit kann ein Cochleaimplantat (Cl) eingesetzt werden. Hier ist intraoperativ das erhöhte Risiko für einen Austritt von Perilymphe bei Eröffnung der Cochlea zu beachten ("Oozer») [223]. Chirurgische Massnahmen am endolymphatischen Sack führen zu keiner Besserung der Symptome, bergen das Risiko einer Ertaubung und sind daher kontraindiziert [154].

\subsubsection{Assoziierte Erkrankungen}

Ein erweiterter vestibulärer Aquädukt findet sich auch bei nichtsyndromalen (DFNB4) und syndromalen genetisch bedingten Schwerhörigkeiten (Pendred-Syndrom) ( $\triangleright$ Tab. 5). Bei der DFNB4 (autosomal-rezessive nicht-syndromale familiäre Schwerhörigkeit mit EVA) liegt in 50-70\% der Fälle eine homozygote Mutation des SLC26A4-Gens vor [201, 224].

Das Pendred-Syndrom [225] ist gekennzeichnet durch die Kombination aus EVA (ggf. mit anderen Innenohrfehlbildungen), progressivem sensorineuralen Hörverlust und einer meist euthyreoten Struma (in 50-80\% der Fälle). Diesem ebenfalls autosomal-rezessiv vererbtem Krankheitsbild liegt in bis zu 90 \% der Fälle eine Mutation des SLC26A4-Gens zugrunde [200, 226]. Mit einer Prävalenz von 7,5-10/10 000 Einwohner gilt es als die häufigste syndromale genetisch bedingte Schwerhörigkeit [202]. Die vestibulären Symptome und Befunde entsprechen denen des EVA.

In der Schilddrüse ist Pendrin am Transport von lodid in das Lumen der Schilddrüsenfollikel beteiligt. Abhängig vom lodgehalt der Nahrung ist die Stoffwechsellage beim Pendred-Syndrom euoder hypothyreot. Regelmässige Ultraschallkontrollen sind notwendig, um eine Grössenprogredienz der Struma bzw. eine maligne Entartung zu einem follikulären Schilddrüsenkarzinom frühzeitig zu erkennen. Ein Humangenetiker sollte für die genetische Diagnostik und Beratung hinzugezogen werden [202].

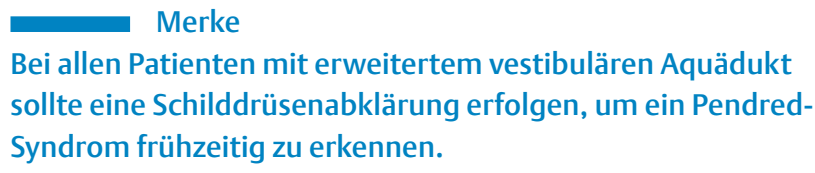

Neben dem Pendred-Syndrom findet sich ein EVA auch bei Innenohrfehlbildungen wie der «incomplete partition type Il» (früher «Mondini-Malformation» genannt) [227] sowie bei komplexen Fehlbildungssyndromen mit Innenohrbeteiligung, wie z. B. dem CHARGESyndrom (siehe hierzu auch den Beitrag von Frau Prof. Dr. Warnecke), branchio-oto-renalem Syndrom, oto-fazio-zervikalem Syndrom, Waardenburg-Syndrom und Noonan-Syndrom [206].

\subsubsection{X-chromosomale familiäre Schwerhörigkeit mit Stapes- Gusher (DFNX2)}

Diese X-chromosomal rezessiv vererbte Erkrankung, die fast ausschliesslich Männer betrifft, ist häufig mit einer Mutation des 
POU3F4-Gens assoziiert ( $\triangleright$ Tab. 5). Ähnlich wie beim EVA handelt es sich hier um die abnorme Erweiterung eines natürlichen neurovaskulären Foramens, nämlich des inneren Gehörganges. Zusätzlich liegt eine inkomplette knöcherne Separation zwischen Cochlea und innerem Gehörgang vor. Dadurch können intrakranielle Druckschwankungen über den erweiterten inneren Gehörgang direkt auf das Innenohr übertragen werden, wo sie zu einer progressiven Schädigung des Sinnesepithels führen. Die Cochlea ist häufig dysplastisch und hat eine korkenzieherartige Gestalt («incomplete partition type II/»). Auch diese Fehbildung führt häufig zur Ertaubung, und bei der Cochleaimplantation ist mit einem PerilymphGusher zu rechnen [77, 129, 130, 223].

\subsubsection{Differenzialdiagnosen von Syndromen des dritten Fensters}

\subsubsection{Vestibuläre Atelektase}

Eine vestibuläre Atelektase wird als zugrundliegende Pathologie bei Patienten mit einer Kombination aus bilateraler Vestibulopathie (Kapitel 4.1) und druck-/lärminduziertem Nystagmus/Schwindel postuliert (Fallbeispiel in \ Abb. 8) [141, 228-230]. Die bilaterale peripher-vestibuläre Unterfunktion wird durch ein kollabiertes membranöses Labyrinth erklärt. Typische Befunde sind hier eine kalorische Unter- bis Unerregbarkeit beider horizontaler Bogengänge sowie ein reduzierter Gain aller Bogengänge im vHIT. Bei manchen Patienten besteht eine Diskrepanz zwischen kalorischer Unerregbarkeit und relativ gut erhaltener hoch-frequenter Bogengangsfunktion im vHIT. Dies wird dadurch erklärt, dass bei kollabiertem Labyrinth durch die niederfrequente kalorische Stimulation keine ausreichende Endolymphströmung für eine Exzitation der vestibulären Haarzellen im horizontalen Bogengang erzeugt werden kann, während der höher-frequente Beschleunigungsreiz beim Kopf-Impuls-Test genügt, um eine Bewegung der Endolymphe auszulösen [228].

Die Provokation von Schwindel durch Druckänderungen des Mittelohres und laute Töne wird auf einen Kontakt des kollabierten membranösen Labyrinthes mit der Stapesfussplatte zurückgeführt. Dieser Befund indiziert, dass bei einer vestibulären Atelektase die bilaterale Vestibulopathie nicht durch einen Funktionsverlust der vestibulären Haarzellen bedingt ist, sondern durch eine mechanische Ursache, welche der Signaltransduktion in den Haarzellen vorausgeschaltet ist, wie zum Beispiel einen Kollaps des häutigen Labyrinthes [228].

Bis vor kurzem war unklar, ob die klinische Kombination aus druck-/lärminduziertem Schwindel und bilateraler Vestibulopathie tatsächlich dem histopathologischen Befund einer vestibulären Atelektase entspricht [231], wie sie erstmals von Merchant und Schuknecht 1988 beschrieben wurde [232]. Durch Fortschritte in der hochauflösenden MRT-Bildgebung des Schläfenbeins ist es jedoch in den letzten Jahren gelungen, den kollabierten Endolymphschlauch in 3D-FLAIR-Sequenzen vier Stunden nach intravenöser Gadoliniumgabe darzustellen, wobei ein- und beidseitige Atelektasen identifiziert wurden [233-235].

Die therapeutischen Optionen bei diesem Krankheitsbild sind sehr begrenzt. Wie bei den Syndromen des dritten Fensters ist es für den Patienten häufig schon beruhigend zu wissen, wie die Symptome zustande kommen. Auslösende Trigger sollten gemieden werden. Als ultima ratio kann - abhängig vom Ausmass der bilateralen Vestibulopathie - eine intratympanale Gentamicingabe diskutiert werden [228]. Die physiotherapeutische Behandlung der bilateralen Vestibulopathie erfolgt wie in Kapitel 4.1.5 beschrieben.

\subsubsection{Andere Differenzialdiagnosen}

Generell kann ein druck- oder lärminduzierter Schwindel/Nystagmus bei allen Erkrankungen des Mittel- und Innenohres ausgelöst werden, bei denen es zu einem direkten Kontakt zwischen dem membranösen Labyrinth und der Stapesfussplatte kommt [140]. Hierzu zählen insbesondere entzündliche Ursachen, wie aus der Erstbeschreibung des Hennebert-Zeichens bei Patienten mit Syphilis hervorgeht [157], Fehlbildungen des Mittelohres oder postoperative/-traumatische Vernarbungen zwischen Stapesfussplatte und Vestibulum [236, 237].

Manchmal berichten auch Patienten mit einem M. Menière von kurzen druck- und lärminduzierten Schwindelsensationen. Dies wird dadurch erklärt, dass das häutige Labyrinth des Vestibulums aufgrund des Endolymphhydrops so stark dilatiert ist, dass es temporär in Kontakt mit der Stapesfussplatte kommt. Steigt der Mit-
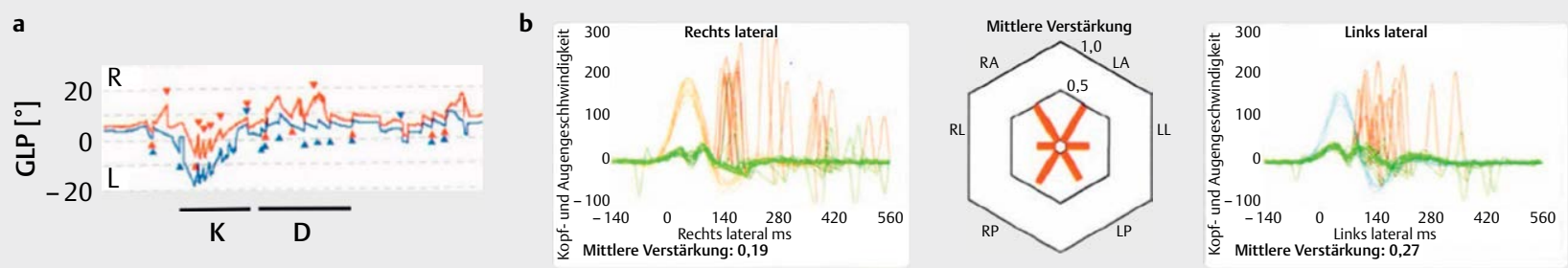

- Abb. 8 Vestibuläre Atelektase. Der 51-jährige Patient beklagte einen kurzfristigen Drehschwindel beim Schnäuzen, welcher ohne erinnerliche Auslöser aufgetreten sei. Auf Nachfrage gab er an, dass er schon immer Schwierigkeiten gehabt habe, beim Gehen im Dunkeln das Gleichgewicht zu halten. a Videookulographie beider Augen (rechts: rot, links: blau) bei Kompression (K) und Dekompression (D) eines Politzer-Ballons im linken Gehörgang. Y-Achse: Geschwindigkeit der langsamen Nystagmusphase (GLP). Hier zeigt sich ein Linksnystagmus bei Kompression (=Exzitation der Haarzellen des horizontalen Bogenganges) und ein Rechtsnystagmus bei Dekompression (= Inhibition). b Video-Kopf-Impuls-Test (vHIT): Im Hexaplot (Mitte) demarkiert sich ein reduzierter Gain für alle sechs Bogengänge, passend zu einer bilateralen Vestibulopathie (BVP) mit Gangunsicherheit im Dunkeln. Exemplarisch sind die Ergebnisse für die beiden horizontalen (=lateralen) Bogengänge mit reduziertem Gain (rechts: 0,19; links: 0,27) und Korrektursakkaden dargestellt. In der kalorischen Prüfung bestand eine Unerregbarkeit beider horizontalen Bogengänge (nicht dargestellt). Details s. Kap. 3.2.8.1. 
telohrdruck an (z. B. beim Valsalva-Manöver) oder wird die Stapesfussplatte durch laute Töne ausgelenkt, so wird die Druckwelle direkt auf die Endolymphe des Vestibulums übertragen, wo sie über eine passagere Exzitation der vestibulären Haarzellen zu kurzzeitigem Schwindel führt [238-240].

\subsection{Positionsabhängiger Schwindel für Sekunden bis Minuten}

\subsubsection{Seltene Varianten des benignen paroxysmalen} Lagerungsschwindels (BPLS)

Der benigne paroxysmale Lagerungsschwindel (BPLS) ist mit einer Lebenszeitprävalenz von 2,4\% eine der häufigsten peripher-vestibulären Erkrankungen [2, 241], in über 90 \% der Fälle dislozieren die Otolithen in den langen Schenkel des posterioren oder horizontalen Bogenganges. Daneben gibt es auch seltene Manifestationen, wie z. B. eine Canalolithiasis des anterioren (= superioren) Bogenganges (a-BPLS), welche bei ca. $3 \%$ der Patienten mit BPLS beobachtet wird $[242,243]$. Des Weiteren wurden auch klinische Präsentationen beschrieben, welche durch die Dislokation von Otolithen in den kurzen Schenkel des posterioren oder horizontalen Bogenganges oder in das Crus commune des posterioren Bogenganges erklärt werden können. Eine ausführliche Übersicht über Symptome, Nystagmusmuster und geeignete therapeutische Manöver findet sich bei [50].

Für alle Formen des BPLS gelten die drei Ewald'schen Gesetze [46]: der Nystagmus schlägt in der Ebene des betroffenen Bogenganges (Erstes Ewald'sches Gesetz), und aus der Nystagmusrichtung lässt sich auf eine Exzitation bzw. Inhibition des betroffenen Bogenganges schliessen (Zweites und drittes Ewald'sches Gesetz) ( $\triangleright$ Abb. 1). Gerade bei seltenen Formen des BPLS ist eine videookulographische Aufzeichnung des Nystagmus in unterschiedlichen Blickrichtungen empfehlenswert, um die einzelnen Nystagmuskomponenten und damit die betroffenen Bogengänge zu identifizieren [49, 244].

Beim sog. BPLS Typ II zeigt sich meist kein Nystagmus im DixHallpike-Manöver, jedoch kommt es beim Aufsitzen von der betroffenen Seite zu einer heftigen Pro- und Retropulsion des Rumpfes. Die Symptomatik schwächt sich bei der wiederholten Durchführung der Dix-Hallpike-Manöver ab. Als zugrundeliegende Pathologie wird eine Dislokation von Otolithen in den kurzen Schenkel des posterioren Bogenganges angenommen [245, 246].

\subsubsection{Seltene Differenzialdiagnosen eines BPLS}

Hinter dem «Hufgeklapper» eines BPLS können sich aber auch einige «Zebras» mit ähnlicher klinischer Präsentation verbergen. Generell sollte die Diagnose eines BPLS in folgenden Fällen kritisch hinterfragt werden [50, 247]:

- Die Nystagmusrichtung korreliert nicht mit der Ebene des

Bogenganges, welcher durch ein bestimmtes Lagerungsma-

növer stimuliert bzw. gehemmt wird.

- Der Nystagmus ist rein torsional oder vertikal.

- Die Eigenschaften des Nystagmus sind uncharakteristisch für einen BPLS, z. B. keine Latenz, fehlendes Crescendo-Decrescendo-Muster, keine Nystagmusumkehr („unwinding nystagmus“) beim Hinlegen/Aufsetzen für die vertikalen Bogengänge bzw. Drehung von der rechten auf die linke Körperseite für die horizontalen Bogengänge.
- Eine wiederholte korrekte Durchführung der Repositionsmanöver für den vermeintlich betroffenen Bogengang führt zu keiner Besserung der Symptomatik.

- Nystagmus- und Schwindelintensität bei den Lagerungsmanövern stimmen nicht überein.

- Auf dem vermeintlich betroffenen Ohr liegt zusätzlich eine Hörminderung vor (siehe auch Kapitel 2.2.1 und 3.2.6).

In diesen Fällen sollte eine weiterführende audiovestibuläre Diagnostik und eine Bildgebung von Gehirn und Schläfenbein durchgeführt werden (CT oder MRT je nach Symptomatik). > Tab. 4 fasst die wichtigsten Erkrankungen zusammen, welche einen BPLS imitieren können.

\subsection{Spontaner episodischer Schwindel (Stunden bis Tage)}

Bei den folgenden Erkrankungen handelt es sich um «Zebras», die häufig die klinische Präsentation eines M. Menière imitieren, d. h. spontan auftretende, rezidivierende (audio-)vestibuläre Funktionsstörungen für Stunden (bis Tage). In der Frühphase ist ein episodischer bis fluktuierender Verlauf typisch, im Langzeitverlauf kommt es häufig zu einer progressiven Verschlechterung der vestibulären Funktion bis hin zu einem chronischen vestibulären Syndrom (Kapitel 4). Neben den im folgenden Abschnitt genannten Erkrankungen (Intralabyrinthäre Schwannome, Tumoren des endolymphatischen Sacks, Autoimmunerkrankungen des Innenohres) kann sich auch ein erweiterter Aqueductus vestibuli (Kapitel 3.2.6) mit diesem klinischen Bild präsentieren.

\subsubsection{Intralabyrinthäres Schwannom}

\subsubsection{Epidemiologie und Klassifikation}

Diese Sonderform der Schwannome des achten Hirnnerven - auch primäres Innenohrschwannom genannt [248] - geht von SchwannZellen des N. vestibularis bzw. cochlearis innerhalb des Labyrinths aus [249, 250]. Wenngleich erstmals 1917 beschrieben [251], galten diese gutartigen Innenohrtumore lange als Rarität. Mit den verbesserten Möglichkeiten der Innenohr-Bildgebung im MRT [23] und dem erhöhten Bewusstsein für ihre Existenz werden intralabyrinthäre Schwannome (ILS) in den letzten Jahren häufiger diagnostiziert [252]. Neuere Studien gehen davon aus, dass ihre jährliche Inzidenz bei > 1/100 000 liegt [253] und sie 10\% aller Schwannome des achten Hirnnerven ausmachen [254]. Bislang wurden etwa 500 Fälle in der Literatur beschrieben [255]. Die Klassifikation intralabyrinthärer Schwannome erfolgt auf der Basis ihrer Lokalisation und Ausdehnung. Meist werden die Klassifikationssysteme nach Kennedy [256], Salzman [257] und Van Abel [248] angewandt. Intracochleäre Schwannome stellen mit ca. 50 \% die häufigste Gruppe der intralabyrinthären Schwannome dar. Bilaterale ILS wurden sowohl bei Patienten mit Neurofibromatose Typ II [258] als auch sporadisch beschrieben [259].

\subsubsection{Klinische Präsentation und Bildgebung}

Das häufigste Symptom ist der einseitige Hörverlust, der bei $99 \%$ der Patienten gefunden wird. Abhängig von der Lokalisation des Tumors kommen Schwindel und Gleichgewichtsstörungen hinzu. Der zeitliche Verlauf der cochleo-vestibulären Symptome ist äusserst variabel: diese können episodenhaft, fluktuierend oder pro- 
gressiv auftreten. In einer Studie erhielten 39\% der Patienten mit ILS anfänglich die Diagnose eines M. Menière [248], da sich beide Erkrankungen mit einer ähnlichen Symptomatik präsentieren. Die fluktuierende Symptomatik bei ILS deutet auf einen sekundären Endolymphhydrops hin. Hierfür spricht neben den ähnlichen audiovestibulären Untersuchungsbefunden bei beiden Krankheitsbildern [260] auch der kürzlich beschriebene Nachweis eines endolymphatischen Hydrops im Schläfenbein-MRT bei Patienten mit ILS $[261,262]$.

\section{TIPP FÜR DIE PRAXIS}

Bei allen Patienten mit einseitigem audiovestibulären Funktionsverlust (fluktuierend oder stabil) sollte eine MRT-Bildgebung des Schläfenbeins mit der expliziten Fragestellung nach einem intralabyrinthären Schwannom gemäss den Empfehlungen der Deutschen Röntgengesellschaft erfolgen [116].

Dabei ist es essentiell, bei der MRT-Bildgebung dezidiert nach einem ILS zu fragen, da die Gefahr besteht, dass dieses aufgrund der kleinen Grösse und der ungewöhnlichen Lokalisation übersehen wird, selbst wenn es auf den MRT-Bildern dargestellt wird [254, 263]. So erklärt sich auch die lange Latenz (durchschnittlich 7 Jahre) vom Auftreten der ersten Symptome bis zur Stellung der Diagnose [248]. Neben einem M. Menière können ILS auch das klinische Bild eines BPLS imitieren (siehe Kapitel 3.3.2 und $\triangleright$ Tab. 4). Eine wichtige Differenzialdiagnose intravestibulärer Raumforderungen sind neben Blutungen (Kap. 2.2.2) und Fibrosen auch die sehr seltenen intralabyrinthären Lipome [264, 265].

\subsubsection{Therapie}

Lange wurden Patienten mit ILS überwiegend mit einer «Wait-andtest-and-scan»-Strategie behandelt, besonders wenn noch ein funktionierendes Gehör auf der betreffenden Seite vorlag. Mit der Weiterentwicklung der mikrochirurgischen Techniken und der Möglichkeit der Cochlea-Implantation hat hier in den letzten Jahren ein Paradigmenwechsel stattgefunden. Gerade bei den häufigen intracochleären Schwannomen wird eine frühzeitige Tumorresektion (partielle, subtotale oder «near-total» Cochleoektomie je nach Tumorausdehnung) mit simultaner Cochlea-Implantation empfohlen [252, 254, 266]. Bei der Verwendung von perimodiolären Elektrodenträgern, welche mittels «cartilage-in-perichondrium-bed»-Technik bei der Cochlea-Rekonstruktion zusätzlich an den Modiolus angenähert werden, lassen sich sehr gute Ergebnisse in der Hörreha-
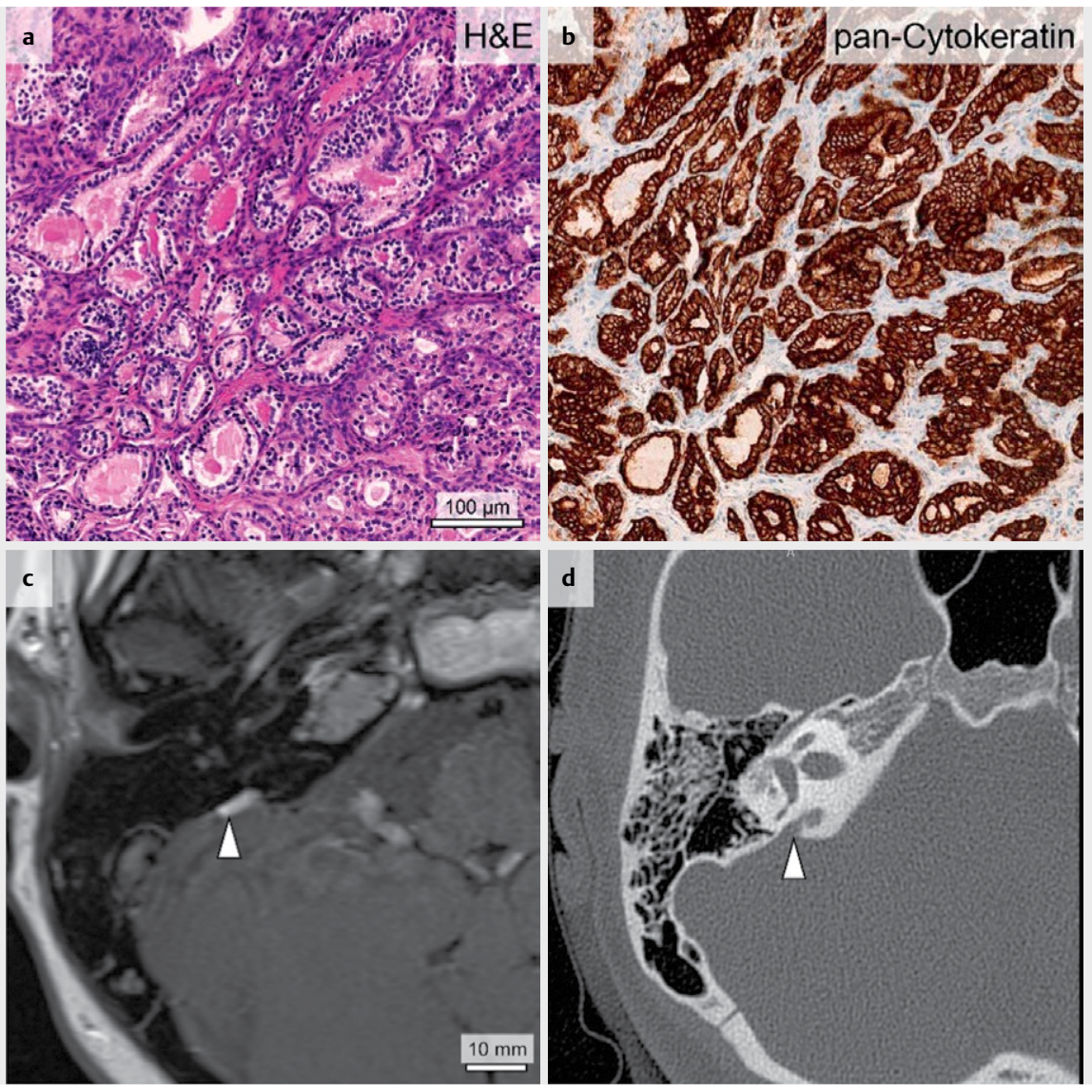

- Abb. 9 Tumor des endolymphatischen Sacks (ELST). a und b Typisches papilläres Muster in der Hämatoxylin-Eosin (H\&E)- sowie in der pan-Cytokeratin-Färbung am histologischen Präparat. In der präoperativen Bildgebung des Patienten zeigte sich in der T2-Wichtung des cMRT ein zystischer Tumor des rechten Saccus endolymphaticus (Pfeilspitze in c) mit Aufweitung und Arrosion des Aqueductus vestibuli im HRCT Schläfenbein (Pfeilspitze in d). Abbildung mit freundlicher Genehmigung von Dr. David Bächinger, Zürich. 
bilitation mit Cl erreichen [267, 268]. Des Weiteren ist es möglich, bei der Cochleoektomie die Bogengangsfunktion zu erhalten [263].

Berichte über stereotaktische Bestrahlung intralabyrinthärer Schwannome sind selten [269, 270]. Hier ist zu beachten, dass die Spiralganglienneurone im Bestrahlungsfeld liegen und damit das Risiko ihrer Degeneration mit neuraler Ertaubung besteht [262].

\subsubsection{Tumoren des endolymphatischen Sacks}

\subsubsection{Klinische Präsentation und Bildgebung}

Hierbei handelt es sich um niedrig-maligne papilläre Neoplasien («low-grade adenocarcinoma»), welche vom Epithel des Ductus/Saccus endolymphaticus im Bereich des knöchernen Aqueductus vestibuli ausgehen ( $\triangleright$ Abb. 9) [271-273]. Die Tumoren wachsen lokal destruktiv und infiltrierend, metastasieren aber äusserst selten (insgesamt drei Fallbeschreibungen mit zerebellären bzw. spinalen Metastasen in der Literatur) $[274,275]$. Aktuell sind in der Literatur weniger als 200 Fälle von Tumoren des endolymphatischen Sacks (ELST) beschrieben [276]. Die Symptomatik mit fluktuierenden bzw. chronisch-progredienten einseitigen audiovestibulären Symptomen ähnelt der des M. Menière [273, 275, 277]. Entsprechend wurde auch bei Patienten mit ELST ein - a.e. sekundärer - Endolymphhydrops im Innenohr-MRT nachgewiesen [278]. Als Ursache für den Hydrops wird neben einer mechanischen Blockade des Endolymphabflusses auch eine Hochregulation von Typ2-Vasopressin-Rezeptoren im endolymphatischen Sack diskutiert [279].

ELST stellen sich im Schläfenbein-MRT heterogen dar. Die soliden Anteile der gefässreichen, gelappten Tumoren reichern Kontrastmittel an. Einblutungen zeigen sich bereits in der T1-Wichtung ohne Kontrastmittel hyperintens, zystische Komponenten erscheinen in der T2-Wichtung hyperintens ( $\mathbf{A} \mathbf{b} \mathbf{b}$. 9). Eine Tumorausbreitung bis in den Kleinhirnbrückenwinkel und das Kleinhirn ist möglich. Ergänzend zum MRT sollte auch eine hochauflösende CTBildgebung bzw. ein DVT des Schläfenbeins zur Beurteilung der knöchernen Destruktionen durchgeführt werden. Hier zeigen sich typischerweise «Mottenfrass-ähnliche» Läsionen [275].

\subsubsection{Assoziation mit dem Von-Hippel-Lindau-Syndrom}

In ca. 30 \% der Fälle stellen ELST die Erstmanifestation eines VonHippel-Lindau-Syndroms (vHL) dar [280]. Dieser seltenen Erkrankung (geschätzte Prävalenz: 1:39000) mit autosomal-dominantem Erbgang liegt eine Mutation im VHL-Gen (Chromosom 3p25.3) zugrunde ( $\triangleright$ Tab. 5) [224]. Sie äussert sich neben ELST in multiplen weiteren Tumoren, wie z. B. Hämangioblastomen des ZNS und der Retina, Phäochromozytomen der Nebenniere, klarzelligen Nierenzellkarzinomen und endokrinen Tumoren des Pankreas [281].

\section{TIPP FÜR DIE PRAXIS}

Bei der Diagnose eines Tumors des endolymphatischen Sacks sollte immer eine weiterführende Diagnostik bezüglich Von-Hippel-Lindau-Syndrom erfolgen.

Diese umfasst neben einem MRT des Schädels, des Rückenmarks und des Abdomens eine ophthalmologische Untersuchung und eine ge- netische Testung auf eine Mutation im VHL-Gen [275]. Eine gross angelegte europäische Registerstudie von 2016 zeigte, dass 3,6\% der Patienten mit vHL-Syndrom Tumoren des endolymphatischen Sacks aufweisen. In $20 \%$ der Fälle treten diese beidseitig auf [280].

\subsubsection{Therapie}

Die Therapie der Wahl ist eine komplette Resektion des Tumors, welche je nach Ausdehnung über einen translabyrinthären, retrolabyrinthären, retrosigmoidalen oder subtemporalen Zugang erfolgen kann [282]. Bei frühzeitiger Resektion besteht die Möglichkeit des Hörerhaltes. Falls eine komplette Resektion nicht möglich ist, wird eine adjuvante Radiotherapie empfohlen. Mit diesem Konzept wird in der Regel eine langjährige Rezidivfreiheit erreicht. Eine Bestrahlung alleine führt zu keiner Kontrolle des Tumorwachstums $[275,276]$. Es liegen einzelne Fallberichte über eine Tumorreduktion mit Tyrosinkinase-Inhibitoren im Sinne einer Salvage-Therapie bei nicht-operablem Tumor vor [283].

\subsubsection{Autoimmunerkrankungen des Innenohres}

Autoimmunbedingte Erkrankungen des Innenohres (autoimmune inner ear disease, AIED) sind mit einer geschätzten jährlichen Inzidenz von <5/100 000 sehr selten. Die Dunkelziffer ist wahrscheinlich höher aufgrund der klinischen Heterogenität des Krankheitsbildes und des Fehlens gesicherter diagnostischer Marker. Insgesamt wird geschätzt, dass ca. 1 \% aller cochleovestibulären Erkrankungen autoimmunen Ursprungs sind [284]. Um bei dem eingangs erwähnten Bild von «Pferden» und «Zebras» zu bleiben, handelt es sich bei der AIED um einen ganzen «Zoo» an Erkrankungen, deren systematische Abhandlung den Umfang dieses Artikels sprengen würde. Der folgende Abschnitt soll vielmehr die Sensibilität des HNO-Arztes für «red flags» dieses Krankheitsbildes schärfen und ihn bei der Durchführung einer Basisdiagnostik unterstützen. In 15-30\% der Fälle tritt die AIED als Manifestation einer systemischen Autoimmunerkrankung auf (sekundäre AIED) [285], die wichtigsten Ursachen sind in \ Tab. 7 zusammengefasst. Das Susac-Syndrom wird im Beitrag von Frau Prof. Dr. Warnecke [286] detailliert vorgestellt.

\subsubsection{Definition}

Wie so häufig für Erkrankungen des Innenohres, ist über die audiologischen Manifestationen von AIEDs weitaus mehr bekannt als über die vestibulären. Dies spiegelt sich auch in den verschiedenen Definitionen von AIED wider. Das zentrale diagnostische Kriterium ist hier ein fluktuierender, progressiver sensorineuraler Hörverlust über Wochen bis Monate. Die Progression verläuft zu langsam für einen «Hörsturz» (also > 72 h) und zu schnell für eine Presbyakusis. Als Faustregel gilt, dass ein bilateraler sensorineuraler Hörverlust von mindestens $30 \mathrm{~dB} \mathrm{nHL}$ in einer Frequenz vorliegen sollte, welcher innerhalb von drei Monaten zunimmt (um mind. $15 \mathrm{~dB}$ in einer Frequenz oder mind. $10 \mathrm{~dB}$ in zwei benachbarten Frequenzen) [284, 287]. Ausserdem darf sich der Hörverlust nicht besser durch eine andere Ursache erklären lassen (z. B. Lärmschaden, ototoxische Substanzen) [285, 288].

In ca. $50 \%$ der Fälle beginnt der Hörverlust einseitig, im weiteren Verlauf entwickelt sich nahezu immer eine bilaterale sensorineurale Hörminderung, welche nicht unbedingt symmetrisch sein muss [285]. In der Literatur wird eine begleitende vestibuläre Funk- 
tionsstörung mit ca. $50 \%$ angegeben, klare diagnostische Kriterien wie für die Hörminderung fehlen allerdings bislang [285].

Gerade im frühen Stadium ist ein fluktuierender Verlauf der audiovestibulären Funktionsstörung wie beim M. Menière typisch, audiologische und vestibuläre Symptome/Zeichen können hierbei auch getrennt voneinander auftreten [289]. Insbesondere bei dem klinischen Bild eines bilateralen M. Menière sollte differenzialdiagnostisch eine AIED erwogen werden [285, 287].

\subsubsection{Diagnostisches Procedere}

Anamnese und klinische Untersuchung Neben der zeitlichen Dynamik der audiovestibulären Symptomatik sollte hier insbesondere auf mögliche andere Manifestationen von Autoimmunerkrankungen im HNO-Bereich geachtet werden (siehe $>$ Tab. 7 und den Beitrag von Frau Dr. Weiss [128]). Bei der Systemanamnese sollte gezielt nach ophthalmologischen und neurologischen Symptomen gefragt werden ( $\triangleright$ Abb. 10). Autoimmunerkrankungen, welche Gehirn, Auge und Ohr betreffen können, werden unter dem Überbegriff «Brain-Eye-Ear-Syndromes» (BEE) zusammengefasst ( $\triangleright$ Tab. 7) [290]. Die Systemanamnese wird abgerundet durch Fragen nach Symptomen im Bereich des Gastrointestinaltraktes (z. B. bei M. Crohn, Colitis ulcerosa, Zöliakie) [291-293], des Bewegungsapparates (z. B. rheumatoide Arthritis), der Niere (ANCA-positive Vaskulitiden) und der Schilddrüse (Autoimmunthyreoiditis) [285].

Audiovestibuläre Diagnostik Im Reintonaudiogramm zeigt sich typischerweise eine uni- oder bilaterale sensorineurale Schwerhörigkeit. Eine zusätzliche Schallleitungskomponente ist möglich, z. B. bei einer chronischen Otitis media im Rahmen ANCA-assoziierter Vaskulitiden, einer Gehörknöchelchenankylose bei rheumatoider Arthritis oder einer Tubenfunktionsstörung bei Relapsing Polychondritis [285, 294]. Der Verlauf der Hörschwelle eignet sich zum Monitoring der Krankheitsaktivität und des Therapieerfolgs (siehe Kapitel 3.4.3.3). Die Messungen kann der Patient mithilfe von Tabletbasierten Hörtests inzwischen selbständig zu Hause durchführen [289, 295].

Auch wenn die Patienten nicht über subjektive Schwindelsymptome berichten, sollte im Rahmen der AIED-Abklärung immer eine Fünf-Rezeptor-Diagnostik der Gleichgewichtsorgane durchgeführt werden, um die Beteiligung der einzelnen Endorgane an der AIED zu bestimmen. Aufgrund der hohen Test-Retest-Reliabilität sind diese Untersuchungen zum Monitoring der vestibulären Funktion im Verlauf der Erkrankung geeignet - sozusagen als eine Art «Rein- tonaudiogramm» des Gleichgewichtsorgans [289]. Die Sammlung dieser Daten an grossen Patientenpopulationen stellt ausserdem eine wichtige Voraussetzung für eine eigenständige Definition autoimmunbedingter vestibulärer Erkrankungen dar (s. oben) und erlaubt eine Quantifizierung des Therapieerfolges [296].

Bildgebung Bei Verdacht auf eine AIED sollte immer ein MRT von Gehirn und Schläfenbein angefertigt werden, um andere Erkrankungen mit ähnlicher Symptomatik auszuschliessen (z. B. Vestibularischwannome, intralabyrinthäre Schwannome, Tumoren des endolymphatischen Sacks, multiple Sklerose) und um weitere Hinweise auf ein mögliches «Brain-Eye-Ear»-Syndrom zu erhalten (siehe - Tab. 7). Des Weiteren wird bei Autoimmunerkrankungen mit ZNS-Beteiligung auch häufig eine Kontrastmittelanreicherung im Bereich des N. vestibulocochlearis und der basalen Meningen beobachtet [285, 288, 290, 297, 298].

Labordiagnostik Hier gibt es keine allgemein gültigen Leitlinien. In der klinischen Praxis hat sich die Bestimmung der Parameter aus $\triangleright$ Tab. 8 als Basisdiagnostik bewährt (basierend auf [284, 287290]). Diese kann abhängig vom klinischen Bild und bekannten Vorerkrankungen modifiziert werden. Hier ist - wie auch bei der weiteren Diagnostik und Therapie - die enge Zusammenarbeit mit einem Immunologen zu empfehlen [285].

Infektiöse Erkrankungen mit neurotropen Bakterien oder Viren können einen ähnlichen klinischen Verlauf wie eine AIED zeigen. Da sie kausal behandelbare Ursachen eines audiovestibulären Funktionsverlustes darstellen, sollte trotz ihrer Seltenheit die entsprechende Serologie im Rahmen einer AIED-Abklärung bestimmt werden (siehe Kapitel 2.2.3 und [103]).

Ophthalmologische und neurologische Mitbeurteilung Ähnlich wie bei der neurootologischen Untersuchung sind auch bei den AIEDs die Augen das «Fenster zum Ohr» [289]. Während die entzündlichen Läsionen des Innenohres äusserlich nicht sichtbar sind, kann der Ophthalmologe diese im Bereich des Auges erkennen ( $\triangleright$ Abb. 10), und damit entscheidende Hinweise für das Vorliegen einer AIED im Sinne eines BEE-Syndroms geben.

Abhängig von der Symptomatik sollte auch ein in Autoimmunerkrankungen des ZNS erfahrener Neurologe mit hinzugezogen werden. Dieser entscheidet über weitere Zusatzdiagnostik wie z. B. eine Lumbalpunktion (Nachweis von oligoklonalen Banden, intrathekaler Antikörperproduktion, Antikörper gegen neurotrope Bakterien und Viren, Tumorzellen bei Meningeosis carcinomatosa) und
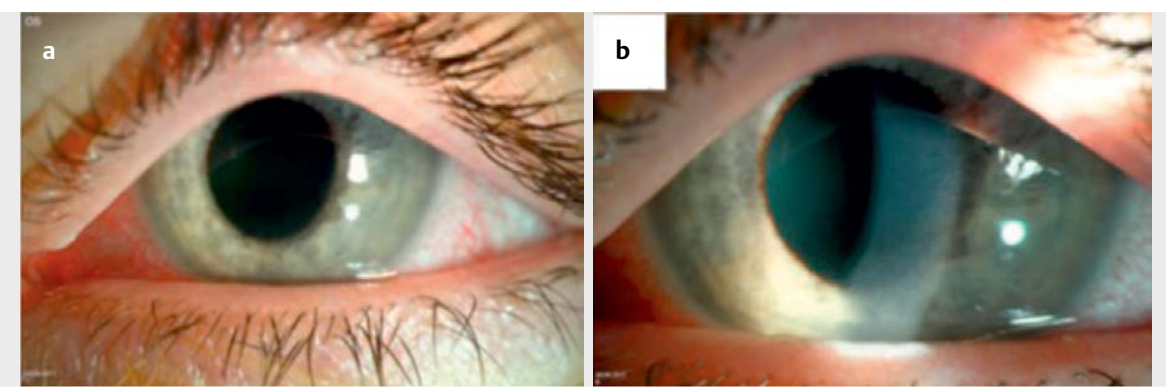

- Abb. 10 Ophthalmologische Befunde bei Cogan-Syndrom. a Gerötete Konjunktiven als Zeichen einer Konjunktivitis. b In der Spaltlampenuntersuchung zeigen sich Zellen in der Vorderkammer des Auges als Hinweis auf eine Uveitis. Abbildung mit freundlicher Genehmigung von Dr. Elias Flockerzi, Homburg/Saar. 
> Tab. 7 Differenzialdiagnose von Autoimmunerkrankungen mit Beteiligung des Innenohres, der Augen und des Gehirns («Brain-Eye-Ear»-Syndrome, nach [287, 289, 290]). Für Details zu den Laborbefunden siehe Tab. 8.

\begin{tabular}{|c|c|c|c|}
\hline & Diagnose & Besonderheiten/ured flags" & Pathognomonische Zusatzuntersuchungen \\
\hline \multirow[t]{5}{*}{ Vaskulitis [294] } & Cogan-Syndrom [390-392] & $\begin{array}{l}\text { Trias: Schwindel, Hörminderung und «rotes } \\
\text { Auge» }\end{array}$ & $\begin{array}{l}\text { - Ophthalmologische Untersuchung: } \\
\text { interstitielle Keratitis, Uveitis, Konjunktivitis } \\
\text { " Spaltlampenuntersuchung: Zellen in der } \\
\text { Vorderkammer des Auges } \\
\text { ( } \text { Abb. 10) }\end{array}$ \\
\hline & Susac-Syndrom & $\begin{array}{l}\text { Trias: Enzephalopathie, retinale Arterienast- } \\
\text { verschlüsse, Hörminderung }\end{array}$ & $\begin{array}{l}\text { " Schädel-MRT: Schneeball-artige balkennahe } \\
\text { Läsionen (T2), «ausgestanzte» Läsionen im } \\
\text { Balken (T1) } \\
\text { " Fluoreszenzangiographie der Retina: } \\
\text { Gefässverschlüsse }\end{array}$ \\
\hline & \multirow[t]{2}{*}{ ANCA-positive Vaskulitiden } & $\begin{array}{l}\text { Granulomatöse Polyangiitis (GPA, früher } \\
\text { Wegener-Granulomatose): therapieresistente } \\
\text { Otitis media }\end{array}$ & cANCA erhöht \\
\hline & & $\begin{array}{l}\text { Eosinophile Granulomatose mit Polyangiitis } \\
\text { (EGPA, früher Churg-Strauss-Syndrom): } \\
\text { rezidivierende polypöse Sinusitiden }\end{array}$ & pANCA erhöht \\
\hline & M. Behcet & $\begin{array}{l}\text { - Orale und genitale Aphten } \\
\text { - Sinusvenenthrombose (SVT) }\end{array}$ & $\begin{array}{l}\text { " positiver Pathergie-Test } \\
\text { - häufig HLAB51 positiv }\end{array}$ \\
\hline \multirow[t]{8}{*}{ andere } & Sarkoidose [297] & $\begin{array}{l}\text { " Heerfordt-Syndrom: Trias aus Uveitis, } \\
\text { Parotitis, Facilaisparese } \\
\text { " Erythema nodosum }\end{array}$ & $\begin{array}{l}\text { " ACE erhöht } \\
\text { " Thorax-Bildgebung: bihiläre pulmonale } \\
\text { Lymphadenopathie }\end{array}$ \\
\hline & Sjögren-Syndrom & $\begin{array}{l}\text { " «dry eyes, dry mouth» } \\
\text { " erhöhtes Risiko für Lymphome der Parotis }\end{array}$ & $\begin{array}{l}\text { - Anti-Ro, Anti-La erhöht } \\
\text { - Parotis-Ultraschall: «Leopardenfellmuster» }\end{array}$ \\
\hline & $\begin{array}{l}\text { Systemischer Lupus erythemato- } \\
\text { des [393] }\end{array}$ & "Schmetterlings-Erythem" & ANA, Anti-ds-DNA, Anti-SmD erhöht \\
\hline & $\begin{array}{l}\text { Antiphospholipid-Antikörper- } \\
\text { Syndrom (APS) }\end{array}$ & $\begin{array}{l}\text { Rezidivierende Thrombosen } \\
\text { " ZNS: Apoplex, TIA, SVT } \\
\text { " Auge: “Amaurosis fugax”, Gesichtsfeldaus- } \\
\text { fälle } \\
\text { - Uterus: rezidivierende Aborte }\end{array}$ & $\begin{array}{l}\text { Anti-Cardiolipin, Anti-beta-2-Glykoprotein, } \\
\text { Anti-Lupuskoagulans erhöht }\end{array}$ \\
\hline & Vogt-Koyangi-Harada-Syndrom & $\begin{array}{l}\text { Alopezie, Vitiligo (befällt überwiegend } \\
\text { melanozytenreiche Gewebe) }\end{array}$ & $\begin{array}{l}\text { " Fluoreszenzangiographie der Retina: } \\
\text { "Sternenhimmel» } \\
\text { " Optische Kohärenztomographie: subretina- } \\
\text { le Flüssigkeitsansammlung }\end{array}$ \\
\hline & $\begin{array}{l}\text { Relapsing Polychondritis } \\
\text { [394-395] }\end{array}$ & $\begin{array}{l}\text { Beteiligung von Ohr-, Nasen- und Kehlkopf-/ } \\
\text { Trachealknorpel (Ohrmuschelperichondritis, } \\
\text { Otitis externa, Tubendysfunktion, Sattelnase, } \\
\text { Laryngo-Tracheomalazie, subglottische } \\
\text { Stenose) }\end{array}$ & Knorpelbiopsie: entzündliche Infiltrate \\
\hline & Rheumatoide Arthritis & Gehörknöchelchenbeteiligung bei 15 - 20\% & RF, Anti-CCP, ANA erhöht \\
\hline & Hashimoto-Thyreoiditis & Steroidsensitive Enzephalopathie & Anti-TPO erhöht \\
\hline
\end{tabular}

über die Therapie zentraler Manifestationen von BEE-Syndromen [289, 290].

\subsubsection{Therapie}

Eine frühzeitige Therapie einer AIED ist entscheidend, da es sich um potentiell reversible audiovestibuläre Funktionsstörungen handelt. Allerdings existieren wegen der Seltenheit und klinischen He- terogenität des Krankheitsbildes nur wenige klinische Studien mit kleinen Patientengruppen, welche meist nicht randomisiert-kontrolliert sind. Zusätzlich erschweren unterschiedliche Zielparameter die Vergleichbarkeit einzelner Studien. Die Therapie bleibt weiterhin eine Gratwanderung zwischen bestmöglichem Erhalt der audiovestibulären Funktion und Vermeidung von therapiebeding- 
ten Nebenwirkungen. Eine aktuelle Übersicht über die medikamentöse Therapie der AIED findet sich bei [299].

\subsection{Glukokortikoide}

Als «Goldstandard» wird ein Therapieversuch mit systemischen Glukokortikoiden empfohlen (z. B. Prednisolon $1 \mathrm{mg} / \mathrm{kg}$ Körpergewicht p.o. für zunächst vier Wochen) - sofern keine Kontraindikationen vorliegen. Zeigt sich nach den ersten vier Wochen eine Verbesserung der Hörschwelle, wird die gleiche Dosierung solange weitergeführt, bis sich in den monatlich angefertigten Audiogrammen eine Stabilisierung des Hörvermögens demarkiert. Danach wird das orale Prednisolon über einen Zeitraum von acht Wochen ausgeschlichen, bis eine Erhaltungsdosis von $10 \mathrm{mg}$ täglich erreicht ist. Nach insgesamt sechs Monaten Glukokortikoid-Therapie und stabilem Audiogramm kann ein Auslassversuch erfolgen. Flammen die Symptome unter dieser Therapie wieder auf, ist unter Federführung eines erfahrenen Immunologen eine erneute Dosiserhöhung oder die Kombination mit Steroid-sparenden Medikamenten (Immunsuppressiva oder Biologika) zu erwägen (siehe unten).

Zeigt sich dagegen innerhalb der ersten 4 Wochen der Steroidtherapie keine Verbesserung des Hörvermögens, wird das orale Prednisolon innerhalb von 12 Tagen ausgeschlichen. Etwa $70 \%$ der Patienten sprechen positiv auf die erste Gabe systemischer Glukokortikoide an. Allerdings kann sich im weiteren Krankheitsverlauf eine Steroidresistenz entwickeln. Auch dann wird auf Immunsuppressiva oder Biologika zurückgegriffen. Generell gilt, dass bis zur Stabilisierung der Hörschwelle einmal monatlich ein Audiogramm durchgeführt werden sollte, danach alle sechs Monate [285, 288]. Zur Prophylaxe steroidassoziierter Nebenwirkungen wird für die Dauer der systemischen Steroidtherapie die tägliche Einnahme von Vitamin D und Calcium (Osteoporoseprophylaxe) sowie Pantoprazol (Magenulkusprophylaxe) empfohlen, ausserdem 2x wöchentlich (z. B. Samstag und Sonntag) die Einnahme von Sulfamethoxazol/Trimethoprim (Pneumocystis carinii-Prophylaxe).

Bestehen Kontraindikationen gegen eine systemische Glukokortikoidtherapie, kann diese auch intratympanal erfolgen, z. B. 1x wöchentlich im betroffenen Ohr über 2 Monate. In einer Untersuchung an 11 Patienten berichteten $54 \%$ nach intratympanaler Applikation von 6-Methylprednisolon über eine Verbesserung des Hörvermögens und der vestibulären Beschwerden [300] (siehe in diesem Zusammenhang auch [301] zur korrekten Nomenklatur von Glucocorticoiden in der lokalen Innenohrapplikation).

\subsection{Weitere Therapieoptionen}

Abhängig vom Krankheitsverlauf und weiteren Erkrankungen des Patienten können unter Federführung des Immunologen Immunsuppressiva wie Cyclophosphamid, Methotrexat, Azathioprim, Ciclosporin oder Mycophenolat Mofetil unter strenger Kontrolle möglicher Nebenwirkungen eingesetzt werden [285, 288].

In einigen Studien wurden Biologika bei Patienten mit AIED verwendet, wenn es während des Ausschleichens des oralen Steroids zu einem Wiederaufflammen der Symptome kam. Hierzu gehören Anti-TNFo-Antikörper (Golimumab, Infliximab, Etanercept), IL1ßBlocker (Anakinra) und Anti-CD20-Antikörper (Rituximab). Infliximab kann auch intratympanal appliziert werden [302]. Trotz positiver Berichte bei einigen Patienten liegen aktuell noch zu wenige Daten vor, um diese Therapien als primäre Alternative zur systemi- schen Steroidtherapie zu empfehlen. Auch der Stellenwert der Plasmapherese bei AIED ist aktuell noch unklar [284, 285, 288, 299].

Kommt es im Rahmen einer AIED zu einer Ertaubung, so sollte möglichst frühzeitig eine Cochlea-Implantation angestrebt werden, um einer entzündlich bedingten Fibrosierung/Ossifikation der Cochlea zuvor zu kommen [284]. Beim Cogan-Syndrom wurde diese bereits acht Wochen nach Ertaubung beschrieben [303]. Die Therapie einer bilateralen Vestibulopathie erfolgt wie in Kapitel 4.1.5 dargelegt.

\section{Merke}

Die Betreuung von Patienten mit autoimmun bedingten Erkrankungen des Innenohres und "Brain-Eye-Ear»-Syndromen erfordert eine enge Kooperation zwischen HNO-Ärzten, Neurologen, Augenärzten und Immunologen, idealerweise in einer interdisziplinären neuro-oto-immunologischen Sprechstunde.

\section{Chronische vestibuläre Syndrome}

Das CVS ist durch folgende Charakteristika gekennzeichnet [5]:

- anhaltender Schwindel/Gleichgewichtsstörung

- Dauer: Wochen bis Jahre

- Symptome und Befunde einer anhaltenden vestibulären Funktionsstörung (z. B. Oszillopsien, Nystagmus, Gangunsicherheit).

Es ist die gemeinsame Endstrecke zahlreicher akuter und episodischer vestibulärer Syndrome, wenn die peripher-vestibuläre Funktion dauerhaft defekt bleibt. Während eine chronische unilaterale vestibuläre Störung in der klinischen Praxis meist schnell identifiziert wird, stellt die bilaterale Vestibulopathie die diagnostischen Fähigkeiten des behandelnden Arztes häufig auf die Probe [304].

\subsection{Bilaterale Vestibulopathie}

Die bilaterale Vestibulopathie (BVP) ist nicht nur bezogen auf die Gesamtbevölkerung ein seltenes Krankheitsbild (geschätzte Prävalenz von 28/100 000 basierend auf dem «United States National Health Interview Survey» von 2008) [305], selbst in spezialisierten Schwindelambulanzen erhalten nur 0,7-7\% der Patienten diese Diagnose $[68,298]$. Die Seltenheit der Erkrankung und die für eine vestibuläre Symptomatik untypische Befundkonstellation resultieren für die betroffenen Patienten häufig in einer wahren Arzt-Odyssee (durchschnittlich sieben Ärzte) und einer langen Latenz von den ersten Symptomen bis zur korrekten Diagnose (bis zu 15 Jahre) $[306,307]$.

\subsubsection{Symptomatik}

Wie bereits in Kapitel 2.3 erwähnt, unterscheiden sich Symptomatik und klinischer Befunde einer beidseitigen vestibulären Unterfunktion deutlich von der einseitigen Form. Insbesondere beklagen die Patienten meist keinen Schwindel, und sie zeigen - bei einem symmetrischen Funktionsverlust beider Gleichgewichtsorgane keinen Spontannystagmus. Letzterer ist der Ausdruck einer Tonusdifferenz zwischen rechtem und linkem Vestibulariskerngebiet im Hirnstamm, wie sie typischerweise bei einseitigen oder asymme- 
trischen vestibulären Funktionsstörungen auftritt, nicht aber bei einer symmetrischen bilateralen Vestibulopathie [63, 304, 306].

Das Leitsymptom der BVP bei $>90 \%$ der Patienten ist vielmehr ein dauerhaftes Gefühl der Imbalance im Stehen und Gehen, welches im Dunkeln und auf unebenen Unterflächen zunimmt [307309]. Bereits ein kurzes, unbewusstes Schliessen der Augen kann zu plötzlichem Orientierungsverlust mit Sturz führen, wie in der Selbstbeobachtung von Crawford [310] anschaulich illustriert wird.

Im Sitzen und Liegen mit absolut ruhigem Kopf haben die Patienten i.d.R. keine Gleichgewichtsbeschwerden. Allerdings können bereits kleinste Kopfbewegungen (z. B. beim Lesen, Kauen, oder bei Autofahrt über eine holprige Strasse) zu störenden Oszillopsien führen [311]. Der bilaterale Ausfall des vestibulo-okulären Reflexes (VOR, \ Abb. 2 und 3) macht eine Stabilisierung des Blickfeldes bei Kopfbewegung unmöglich. Den Patienten fällt es häufig schwer, dieses ungewöhnliche Symptom zu beschreiben. Dies spiegelt sich unter anderem darin wider, dass die Zahl der Patienten, welche Oszillopsien/ «verschwommenes Sehen» angeben, zwischen einzelnen Studien deutlich variiert (20-98\%) [307, 308, 311, 312]. Wegen der «Wackelbilder» suchen viele Patienten primär den Augenarzt auf, welcher bei seiner Untersuchung am sitzenden Patienten mit ruhigem Kopf meist keine Ursache findet, da in dieser Situation der VOR nicht gebraucht wird.

Die entscheidenden Fragen bei der Anamnese lauten hier:

- Müssen Sie beim Spazierengehen stehenbleiben, um Strassenschilder usw. lesen zu können?

- Ist Ihnen aufgefallen, dass sie beim Spazierengehen die Gesichter entgegenkommender Menschen nicht mehr

erkennen, selbst wenn Ihnen diese gut bekannt sind?

Bejaht der Patient eine dieser beiden Fragen, sollten Sie an eine BVP denken.

Viele Patienten berichten auch über kognitive Einschränkungen. Während bereits länger bekannt ist, dass eine partielle und totale bilaterale Vestibulopathie zu einer Volumenreduktion im Hippocampus, einer Störung der räumlichen Orientierung und des räumlichen Gedächtnisses führen kann [313, 314], zeigen neuere Untersuchungen auch kognitive Störungen in anderen Bereichen, z. B. Aufmerksamkeit, Kurzzeitgedächtnis und exekutiven Funktionen [315, 316]. Die komplexe, facettenreiche Symptomatik der BVP führt bei den Betroffenen zu einer starken Einschränkung der Lebensqualität, insbesondere in Bezug auf Selbständigkeit, soziale Kontakte und Berufstätigkeit [307, 317, 318].

\subsubsection{Klassifikation und Diagnostik}

Für die Diagnose einer «klinisch wahrscheinlichen BVP» nach den Kriterien der Bárány-Gesellschaft muss neben der o.g. typischen Symptomatik mit chronischer Imbalance und/oder Oszillopsien ein beidseits pathologischer klinischer Kopf-Impuls-Test für die horizontalen Bogengänge vorliegen. Die Diagnose einer «klinisch sicheren BVP» erfordert zusätzlich den Nachweis eines beidseits pathologischen horizontalen VOR in der apparativen Zusatzdiagnostik (vHIT oder Kalorik oder Drehstuhlpendelung) [311].

Die Funktion der übrigen Bogengänge und der Otolithenorgane ist in der Definition der Bárány-Gesellschaft bislang nicht berücksichtigt. In aktuellen Studien zeigte sich ein breites Spektrum für die von einer bilateralen Unterfunktion betroffenen vestibulären Endorgane, z. B. eine isolierte Unterfunktion beider posterio- ren Bogengänge [319] oder beider Sakkuli [320, 321]. Hier sind weitere Untersuchungen und Verlaufsbeobachtungen notwendig, um den klinischen Stellenwert dieser Befundkonstellationen zu beurteilen [322].

Bilateral pathologische VEMP-Befunde werden bei $60-80 \%$ der Patienten mit BVP (definiert als bilaterale Unterfunktion der horizontalen Bogengänge) berichtet [319, 323, 324]. Aktuell kommt den VEMPs die Rolle einer Zusatzdiagnostik zur Bestimmung des Ausmasses der peripher-vestibulären Schädigung bei BVP zu. Aufgrund der guten Test-Retest-Reliabilität eignen sie sich zusammen mit dem vHIT zum Monitoring der peripher-vestibulären Funktion im weiteren Krankheitsverlauf [322].

Die Störung des vestibulo-okulären Reflexes lässt sich neben dem vHIT durch die Messung der dynamischen Sehschärfe (dynamic visual acuity, DVA) mit der Sehtest-Tafel bestimmen [311, 325]. Für eine genauere Quantifizierung des DVA-Verlustes stehen computergestützte Messmethoden zur Verfügung [325]. Ein pathologischer Romberg-Test i.S. einer starken Zunahme der Körperschwankung und der Fallneigung mit geschlossenen Augen bzw. auf einer Schaumstoffmatte ist sehr sensitiv für eine BVP. Die Spezifität ist allerdings gering, da auch eine zerebelläre bzw. sensomotorische Ataxie ähnliche Reaktionen hervorrufen [107, 304].

\subsection{3 Ätiologie}

Die Ursachen für eine BVP sind vielfältig ( $>$ Tab. 9). Deren relative Häufigkeit variiert zwischen den Publikationen einzelner Arbeitsgruppen, in 20-50\% der Fälle bleibt die Ätiologie trotz intensiver Suche unklar («idiopathische BVP») [298, 308, 309, 321]. Zusammenfassend können alle Erkrankungen mit einer fluktuierenden/ progressiven Funktionsstörung beider Gleichgewichtsorgane bzw. ihrer Afferenzen zum Vollbild einer BVP führen (siehe vorangehende Kapitel). Daher ist es für die Prognose des Patienten von entscheidender Bedeutung, frühzeitig behandelbare Ursachen zu erkennen und somit einen Progress der Erkrankung aufzuhalten oder im besten Falle eine (Teil-)Erholung der peripher-vestibulären Funktion zu erreichen.

Der zeitliche Verlauf der Erkrankung und das "Muster» in der Fünf-Rezeptor-Diagnostik lassen bereits einige Rückschlüsse auf die Ätiologie der BVP zu. Rezidivierende Schwindelattacken mit der sekundären Entwicklung einer bilateralen vestibulären Unterfunktion finden sich insbesondere bei einem bilateralen $\mathrm{M}$. Menière und bei Autoimmunerkrankungen des Innenohres (Kapitel 3.4.3). Ein langsam progredienter Verlauf wird häufig bei der idiopathischen BVP beobachtet, während toxische und autoimmune Ursachen einen eher schnell-progressiven Verlauf zeigen. Eine BVP in Kombination mit neurologischen Symptomen erfordert die besondere Aufmerksamkeit des behandelnden HNO-Arztes (Kapitel 4.1.3.2) [309].

Im vHIT sind bei infektiösen Ursachen der BVP ( beim CANVAS (cerebellar atrophy, neuronopathy, vestibular areflexia syndrome, Kapitel 4.1.3.2.1) typischerweise alle sechs Bogengänge betroffen, während beim bilateralen M. Menière und bei Aminoglykosid-Toxizität (Kapitel 4.1.3.1.1) die Funktion der anterioren Bogengänge häufig intakt bleibt ( $\mathbf{A} \mathbf{b b}$. 2). Die Gründe hierfür sind noch unklar. Pathologische oVEMPs werden häufiger bei Aminoglykosid-Toxizität als bei bilateralem M. Menière beobachtet. Auch die Zahl der betroffenen Endorgane stellt einen möglichen 
differenzialdiagnostischen Hinweis dar (infektiös: 8,7>Aminoglykoside: 8,0 > M. Menière: 5,5) [319, 324, 326].

Im Folgenden werden einige der Ursachen einer bilateralen Vestibulopathie näher erläutert, die im klinischen Alltag von besonderer Bedeutung sind bzw. zu einem besseren Verständnis der zugrundeliegenden Pathophysiologie dieses Krankheitsbildes beitragen.

\subsubsection{Toxische und metabolische Ursachen}

\subsection{Aminoglykoside}

Die häufigste identifizierbare Ursache einer BVP ist eine Behandlung mit vestibulotoxischen Aminoglykosiden, insbesondere Gentamicin [298, 308, 309] ( A Abb. 2). Grundsätzlich gilt, dass jede Gabe von Gentamicin, egal in welcher Dosierung, in welcher Häufigkeit oder welcher Applikationsform zu einer BVP führen kann [306, 312, 327]. Von den Mutationen der mitochondrialen 12S rRNA, welche für einen ausgeprägten cochleotoxischen Effekt von Aminoglykosiden prädisponieren (z. B. A1555G) [328], wurde bislang keine bei Patienten mit Aminoglykosid-assoziierter oder idiopathischer BVP gefunden [329]. Trotzdem sollte vor Gabe dieser Medikamente nach Möglichkeit eruiert werden, ob eine positive Familienanamnese für Aminoglykosidassoziierte Ototoxizität vorliegt.

Die deletäre Wirkung von Gentamicin auf das vestibuläre Labyrinth ergibt sich aus seinen pharmakologischen und pharmakoki- netischen Eigenschaften. Es schädigt bevorzugt vestibuläre Haarzellen vom Typ I, während die Cochleotoxizität relativ gering ausgeprägt ist [330,331]. Damit entfällt häufig eine subjektiv spürbare Hörminderung als Warnsignal für eine ototoxische Wirkung - sowohl für den Patienten als auch für den behandelnden Arzt [298, 332] (\ Abb. 2). Vestibuläre Haarzellen vom Typ I sind hochspezialisierte Sensoren für schnelle Änderungen der Beschleunigung, z. B. bei schnellen Kopf- und Körperdrehungen [333]. Da die Patienten während einer Behandlung mit systemischem Gentamicin meist schwer krank und bettlägerig sind, fällt die vestibulotoxische Wirkung frühestens nach der ersten Mobilisation auf, meistens aber erst nach Entlassung aus dem Krankenhaus oder viel später. Viele Patienten erinnern sich dann überhaupt nicht mehr an die Gentamicingabe. Daher sollte der Patient bei der Diagnosestellung einer BVP nicht nur allgemein nach der Gabe von Aminoglykosiden in der Anamnese gefragt werden, sondern auch nach längeren Krankenhausaufenthalten wegen einer Infektion oder Sepsis. Meistens bringt erst das gezielte Anfordern der entsprechenden Krankenhausunterlagen Klarheit [306].

Eine weitere Gefahr von Gentamicin besteht in der kumulativen vestibulotoxischen Wirkung. Die Substanz reichert sich über Monate im Innenohr an, bei Meerschweinchen beträgt die Eliminations-Halbwertzeit sechs Monate. So kann sie ihre zerstörerische

> Tab. 8 Labordiagnostik bei V.a. autoimmune Innenohrerkrankung (AIED) (nach [284, 287-290]).

\begin{tabular}{|c|c|c|}
\hline & Untersuchung & Auffällige Befunde/Assoziierte Erkrankungen \\
\hline \multirow[t]{2}{*}{$\begin{array}{l}\text { Screening auf entzündli- } \\
\text { che Erkrankungen }\end{array}$} & $\begin{array}{l}\text { " Grosses Blutbild 1, } \\
\text { " Serum: Leber-, Nierenwerte, Elektrolyte, Eiweiss, fT4, TSH, } \\
\text { Immunglobuline (IgG-Subklassen, IgA, IgM) } \\
\text { " Urinstatus inkl. Eiweiss, Calcium, Albumin }\end{array}$ & $\begin{array}{l}\text { z.B. Entzündungsanämie, erhöhte Immunglobu- } \\
\text { line bei Autoimmunerkrankungen, Hinweise auf } \\
\text { systemische Manifestation von Autoimmuner- } \\
\text { krankungen }\end{array}$ \\
\hline & CRP, BSG ${ }^{2}$ & generell erhöht \\
\hline Komplement & $\mathrm{C} 3 \mathrm{c}, \mathrm{C} 4$ & $\begin{array}{l}\text { generell erniedrigt bei Autoimmunerkrankun- } \\
\text { gen (vermehrter Verbrauch) }\end{array}$ \\
\hline Enzyme & ACE & erhöht bei Sarkoidose \\
\hline \multirow{3}{*}{$\begin{array}{l}\text { Anti-nukleäre Antikörper } \\
\text { (ANAs) }\end{array}$} & ANA & generell erhöht bei Autoimmunerkrankungen \\
\hline & $\begin{array}{l}\text { Anti-ds-DNA } \\
\text { Anti-SmD }\end{array}$ & erhöht bei systemischem Lupus erythematodes \\
\hline & Anti-SSA (Ro)/Anti-SSB (La) & erhöht bei Sjögren-Syndrom \\
\hline \multirow{2}{*}{$\begin{array}{l}\text { Anti-zytoplasmatische } \\
\text { Antikörper (ANCAs)/ } \\
\text { ANCA-assoziierte } \\
\text { Vaskulitiden }\end{array}$} & cANCA (gegen Proteinase 3, PR3) & $\begin{array}{l}\text { erhöht bei Granulomatose mit Polyangiitis } \\
\text { (GPA, früher Wegener-Granulomatose) }\end{array}$ \\
\hline & pANCA (gegen Myeloperoxidase, MPO) & $\begin{array}{l}\text { erhöht u.a. bei mikroskopischer Polyangiitis und } \\
\text { eosinophiler Granulomatose mit Polyangiitis } \\
\text { (EGPA, früher Churg-Strauss-Syndrom) }\end{array}$ \\
\hline \multirow[t]{3}{*}{ Andere Antikörper } & $\begin{array}{l}\text { RF (Rheumafaktor) } \\
\text { Anti-CCP/ACPA (Antikörper gegen das cyclische citrullinierte Peptid) }\end{array}$ & erhöht bei rheumatoider Arthritis \\
\hline & $\begin{array}{l}\text { Anti-Cardiolipin } \\
\text { Anti-beta-2-Glykoprotein } \\
\text { Anti-Lupuskoagulans }\end{array}$ & $\begin{array}{l}\text { erhöht bei Antiphospholipid-Antikörper-Syn- } \\
\text { drom (APS) }\end{array}$ \\
\hline & Anti-TPO (Thyreoperoxidase) & erhöht bei Hashimoto-Thyreoiditis \\
\hline Infektionsserologie & Serologie für Lues, HIV, Borrelien & $\begin{array}{l}\text { Differenzialdiagnose erregerbedingter } \\
\text { Erkrankungen }\end{array}$ \\
\hline
\end{tabular}


Wirkung auch bei normalen Serumspiegeln und noch nach Abschluss der Behandlung entfalten [334]. Weiter ist zu beachten, dass die zusätzliche nephrotoxische Wirkung von Gentamicin die renale Ausscheidung der Substanz verzögern kann und somit die Vestibulotoxizität zusätzlich erhöht. Auch die Kombination mit Vancomycin (Glykopeptid) kann die vestibulotoxische Wirkung von Gentamicin potenzieren [298, 312].

Neben Gentamicin wird auch Tobramycin mit vestibulotoxischen Nebenwirkungen in Verbindung gebracht. Dieses Aminoglykosid wird häufig inhalativ zur Therapie pulmonaler Pseudomonasinfektionen bei Patienten mit Mukoviszidose oder Bronchiektasien verwendet. Auch für die inhalative Anwendung wurden - selbst bei Patienten mit normaler Nierenfunktion - vestibulotoxische Nebenwirkungen berichtet [335-337].

\section{Merke}

Vestibulotoxische Aminoglykoside können in jeder Dosierung, jeder Applikationsform und bei jeder Gabe zu einer bilateralen Vestibulopathie führen - auch bei normalem Serumspiegel und normalem Hörvermögen.

\subsection{Monitoring der Vestibularisfunktion}

Wird die vestibulotoxische Wirkung von Aminoglykosiden frühzeitig erkannt, so kann ein weiterer Progress verhindert werden, z. B. durch eine Umstellung auf ein anderes Antibiotikum, wenn dies aus infektiologischer Sicht möglich ist. Im besten Falle kann sogar eine (Teil-) Remission der Schädigung eintreten, da vestibuläre Haarzellen selbst bei adulten Säugetieren - im Gegensatz zu cochleären Haarzellen eine gewisse Regenerationsfähigkeit aufweisen [338-341].

Zur Minimierung Aminoglykosid-bedingter Vestibulotoxizität ist ein regelmässiges Monitoring der vestibulären Funktion erfor- derlich - während der Antibiotikatherapie und auch in den Monaten danach [312]. Im Gegensatz zu den etablierten Empfehlungen für das audiologische Monitoring cochleotoxischer Substanzen mittels Hochtonaudiometrie und otoakustischer Emissionen wurde das Monitoring vestibulotoxischer Substanzen lange vernachlässigt [342]. Mit dem vHIT und den VEMPs stehen heute effektive Messinstrumente zur Verfügung, eine vestibulotoxische Wirkung in allen vestibulären Endorgane zu detektieren und zu quantifizieren, insbesondere da beide Verfahren überwiegend die Funktion der vestibulären Haarzellen vom Typ I, der Zielstruktur vestibulotoxischer Aminoglykoside, messen [333].

\subsubsection{Neurodegenerative Erkrankungen}

Der Anteil zusätzlicher neurologischer Erkrankungen bei Patienten mit BVP variiert abhängig vom Schwerpunkt eines Schwindelzentrums zwischen 4,5\% (HNO-ärztlicher Schwerpunkt) bis zu 39\% (neurologischer Schwerpunkt) [309, 343]. Für den HNO-Arzt ist es an dieser Stelle wichtig, sich dieser Überlappungen bewusst zu sein und zusätzliche neurodegenerative Erkrankungen zu erkennen. So kann der Patient frühzeitig und mit einer gezielten Fragestellung durch die neurologischen Kollegen mitbetreut werden.

\subsection{CANVAS}

Eine besondere diagnostische Herausforderung stellen Patienten mit einer Kombination aus bilateraler Vestibulopathie, zerebellärem Syndrom und sensorischer Neuro(no)pathie dar, sei es durch eine inzidentelle Kombination (z. B. zerebelläre Atrophie + Gentamicingabe) oder im Rahmen eines CANVAS (cerebellar atrophy, neuronopathy, vestibular areflexia syndrome). Letzteres folgt a.e. einem autosomal-rezessiven Erbgang mit später Manifestation, eine zugrundeliegende genetische Mutation wurde bislang nicht gefun-

> Tab. 9 Ursachen einer bilateralen Vestibulopathie (modifiziert nach [298, 304, 309]).

toxisch (Kap. 4.1.3.1, $>$ Abb. 2)

metabolisch

neurodegenerative Erkrankungen (Kap. 4.1.3.2)

iatrogen (Kap. 4.1.3.3)

genetisch (Kap. 4.1.3.4)

traumatisch

kongenital/syndromal

infektiös (Kap. 2.2.3 und 2.3)

autoimmun (Kap. 3.4.3)

normalerweise unilateral auftretende bilaterale Erkrankungen

neoplastisch

andere
Aminoglykoside (bes. Gentamicin und Tobramycin), Cisplatin, Schleifendiuretika, Salicylate in hohen Dosen (5g/Tag) [396], Penicillin + nicht-steroidale Antiphlogistika [397], Amiodaron [398], Hydroxychloroquin [399], Styren [400], Flugzeugbenzin [401], Kobalttoxikose [402]

Niereninsuffizienz, Vitamin B1-, B6-, B12-/Folsäuremangel [403], Hypothyreose, Diabetes mellitus [404], Alkoholabusus

CANVAS und Differenzialdiagnosen (spinozerebelläre Ataxien, Friedreich-Ataxie, Multisystematrophie, Wernicke-Enzephalopathie) [344, 405], superfizielle Siderose, periphere Polyneuropathien (degenerativ, inflammatorisch, hereditär) [406-408]

bilaterale operative Eingriffe an der lateralen Schädelbasis, z.B. Cochlea-Implantat, Schädelbasistumoren siehe $>$ Tab. 5

Contusio labyrinthi, bilaterale Schläfenbeinfraktur ( $\mathbf{A b b . ~ 3 )}$

Innenohrfehlbildungen z.B. CHARGE-Syndrom, Aplasie der Bogengänge [409-411], erweiterter Aqueductus vestibuli (Kap. 3.2.6, - Abb. 7), prä-/perinatale Infektionen (CMV, Röteln)

Meningitis, Neurosyphilis [103], Neuroborreliose [94], neurotrope Viren (HSV, VZV, CMV, EBV, HIV)

siehe $>$ Tab. 7, [296]

Neuritis vestibularis $[105,106,412]$ (Kap. 2.3), M. Menière [2]

Neurofibromatose Typ II [413], Schädelbasismeningeome, Meningeosis carcinomatosa, Metastasen/ Lymphom im Kleinhirnbrückenwinkel [414]

aseptische Meningitis [415], vestibuläre Atelektase (Kapitel 3.2.8, > Abb. 8), Presbyvestibulopathie [416], Formenkreis der auditorischen Neuropathien, Otosklerose [417] 
den. Die diagnostischen Kriterien finden sich bei Szmulewicz et al. [344].

Jede der drei Krankheitskomponenten per se kann sich in einer Ataxie manifestieren. Daher ist es von entscheidender Bedeutung, bei der klinischen und apparativen Untersuchung auf pathognomonische Zeichen jeder einzelnen Erkrankung zu achten, insbesondere auf eine zerebelläre Okulomotorikstörung (sakkadierte Blickfolge, dysmetrische Sakkaden, Blickrichtungsnystagmus, ReboundNystagmus, Downbeat-Nystagmus, gestörte Fixationssuppression des VOR) [345, 346]. Videobeispiele für diese Befunde finden sich bei [347]. Die BVP lässt sich durch den bilateral gestörten VOR im (Video-)HIT diagnostizieren. Typisch für eine Kombination von BVP und zerebellärem Syndrom ist eine Störung des visuell verstärkten VOR (vVOR) (siehe hierzu das Video in [344]).

\section{TIPP FÜR DIE PRAXIS}

Ein gestörter visuell verstärkter vestibulo-okulärer Reflex ( $v V O R$ ) ist ein typisches Zeichen für eine Kombination aus bilateraler Vestibulopathie und zerebellärem Syndrom.

Die Therapie einer BVP im Rahmen einer neurodegenerativen Erkrankung richtet sich nach der Grunderkrankung. Bei störenden Downbeat-Nystagmen, welche im Gegensatz zur BVP bereits bei komplett ruhigem Kopf Oszillopsien verursachen können, kommen Aminopyridine (Cave: Verlängerung der QTc-Zeit im EKG!), Chlorzoxazon oder Baclofen zum Einsatz [348].

4.1.3.2.2 Superfizielle Siderose Bei diesem äusserst seltenen Krankheitsbild kommt es aufgrund rezidivierender subarachnoidaler Blutungen zu Hämosiderinablagerungen im ZNS, bevorzugt in den Zellen der Glia. Insgesamt ist bisher nur bei ca. 30 Patienten in der Literatur eine vestibuläre Beteiligung bei superfizieller Siderose beschrieben [349]. Neben einem progressiven bilateralen audiovestibulären Funktionsverlust finden sich häufig ein zerebelläres Syndrom und andere neurologische Ausfälle [350, 351]. Die Hämosiderinablagerungen stellen sich besonders deutlich in der Gradienten-Echo-T2-Sequenz ( $2^{*}$ ) als hypointenser Randsaum um die betroffenen Strukturen dar [352]. Bei der Anamneseerhebung sollte insbesondere nach stattgehabten intraduralen Operationen oder Schädel-Hirn-Traumata gefragt werden. Die Symptome der superfiziellen Siderose treten oft erst Jahre später auf. Die kausale Therapie besteht in der Identifikation und Beseitigung der Blutungsquelle zusammen mit den Kollegen der Neurologie und Neurochirurgie [352, 353]. Oft lässt sich jedoch trotz intensiver Suche keine Quelle finden. Der Stellenwert von Eisenchelatoren in der Therapie ist noch unklar [350].

\subsubsection{3 latrogene Ursachen}

Vor operativen Eingriffen an der lateralen Schädelbasis sollte immer eine umfassende, seitengetrennte Prüfung der Vestibularisfunktion mittels Fünf-Rezeptor-Diagnostik erfolgen. Für den seltenen Fall, dass auf der Gegenseite präoperativ eine peripher-vestibuläre Unterfunktion vorliegt (z. B. Vestibularisschwannom mit erhaltener Vestibularisfunktion, aber peripher-vestibulärer Unterfunktion auf der Gegenseite), sollte das therapeutische Konzept individuell im interdisziplinären Team mit Neurochirurgen und Radioonkologen derart modifiziert werden, dass das Risiko für eine postinterventionelle bilaterale Vestibulopathie minimiert wird.

Eine besondere Bedeutung kommt der vestibulären Statusbestimmung bei der Operation der «zweiten Seite» zu, z. B. bei Schädelbasismeningeomen, bilateralen Vestibularisschwannomen oder auch Cochlea-Implantationen [237]. Wenn der Patient nach der Operation der ersten Seite einen peripher-vestibulären Funktionsverlust erlitten hat, welcher anschliessend gut zentral kompensiert wurde, herrscht oft die Meinung vor: «Wenn er beim ersten Mal gut kompensiert hat, wird er es beim zweiten Mal auch tun.». Genau das wird eben nicht passieren, weil beim zweiten Eingriff mit vorbestehendem vestibulären Schaden auf der Gegenseite das für die Kompensation notwendige funktionstüchtige Labyrinth fehlt.

Einen weiteren Sonderfall stellt die operative bzw. destruktive Therapie des M. Menière dar. Innerhalb von 10 Jahren entwickeln bis zu 35 \% der Patienten mit initial unilateralem M. Menière eine bilaterale Erkrankung [2]. Falls bei der zuerst betroffenen Seite eine destruktive Therapie durchgeführt wurde (z. B. intratympanale Gentamicingabe, Labyrinthektomie, Neurektomie des N. vestibularis), kann in diesen Fällen eine BVP resultieren. Der behandelnde HNO-Arzt muss den Patienten bei der Therapieplanung über diese mögliche Entwicklung informieren, um gemeinsam mit ihm im Sinne einer partizipativen Entscheidungsfindung ("shared decision making») einen Kompromiss aus Attackenreduktion und dem Risiko einer BVP zu finden. Eine wichtiges Thema für künftige klinische Studien ist in diesem Zusammenhang die Frage, ob Prädiktoren für die Entwicklung eines beidseitigen M. Menière existieren (z. B. bestimmte Genexpressionsmuster [354, 355], ein endolymphatischer Hydrops auf der (noch) gesunden Gegenseite oder eine bestimmte Morphologie des Aqueductus vestibuli/des endolymphatischen Sacks [356, 357]).

Auch Bestrahlungen der Schädelbasis können vestibulotoxisch wirken. Hier liegen insbesondere über den Langzeitverlauf aktuell noch wenige Daten vor. Daher ist generell zu empfehlen, die Gleichgewichtsfunktion auch in die Planung radiochirurgischer Interventionen der Schädelbasis miteinzubeziehen.

\section{Merke}

Vor Interventionen an der Schädelbasis sollte immer eine seitengetrennte Fünf-Rezeptor-Diagnostik der

Gleichgewichtsorgane durchgeführt werden. Die Ergebnisse sind ein integraler Bestandteil der interdisziplinären Therapieplanung zwischen HNO-Heilkunde, Neurochirurgie und Radioonkologie.

\subsubsection{Genetische Ursachen}

Im Gegensatz zu genetisch bedingten Schwerhörigkeiten (siehe hierzu auch den Beitrag von Frau Prof. Warnecke [286]) ist über genetische Ursachen der BVP bisher nur wenig bekannt ( $>$ Tab. 5). Bei der Kombination aus bilateraler hochgradiger sensorineuraler Schwerhörigkeit, BVP und Retinitis (Retinopathia) pigmentosa (Dystrophie der Stäbchen und Zapfen in der Retina mit Nachtblindheit und Gesichtsfeldeinengung) stellt das Usher-Syndrom eine wichtige Differenzialdiagnose dar. Dieses ist die häufigste Ursache für genetisch bedingte Taub-Blindheit. Je nach klinischem Verlauf werden drei (bei manchen Autoren vier) Untergruppen unterschieden, denen meist Mutationen in neun verschiedenen Genen zu- 
- Tab. 10 Basisdiagnostik bei bilateraler Vestibulopathie (modifiziert nach den Empfehlungen der deutschen Gesellschaft für Neurologie für die Diagnostik der Polyneuropathie [360]).

\section{Grosses Blutbild}

Serum: CRP, BSG, Eiweiss-Immunelektrophorese, ggf. Immunfixation (monoklonale Gammopathie), Elektrolyte, Leber- und Nierenwerte, Glucose/HbA1c (Diabetes mellitus), Vitamin B1, B6, Vitamin B12, Folsäure, CDT (carbohydrate deficient transferrin, erhöht bei chronischem Alkoholkonsum), TSH, fT4

Urin: Urinstatus inkl. Protein (Bence-Jones-Proteine bei monoklonaler Gammopathie)

Serologie: Borrelien, Treponema pallidum, neurotrope Viren (HSV, VZV, CMV, EBV, HIV)

grunde liegen. In über 50 \% der Familien mit Usher-Syndrom Typ I findet sich eine Mutation im Myosin-7A-Gen (MYO7A) [224, 358, 359]. Ähnlich wie bei den Autoimmunerkrankungen sind hier die Systemanamnese und die Zusammenarbeit mit einem Ophthalmologen entscheidend (Kapitel 3.4.3.2). Zusätzlich sollten sich die Patienten und ihre Familien bei einem Humangenetiker für die genetische Diagnostik und Beratung vorstellen.

Weitere Ursachen der BVP sind in ₹ Tab. 9 zusammengefasst.

\subsubsection{Zusatzdiagnostik zur Ursachenabklärung}

Dieser Punkt stellt den behandelnden Arzt häufig vor ein grosses Dilemma: einerseits will er keine behandelbaren Ursachen einer bilateralen Vestibulopathie übersehen, andererseits ergibt auch eine kostenintensive ausführliche Zusatzdiagnostik in bis zu 20-50\% der Fälle keine zugrundliegende Ätiologie.

Im klinischen Alltag hat sich folgendes Vorgehen bewährt (nach [309]):

- Jeder Patient mit BVP sollte eine Reintonaudiometrie und ein Schädel-/Schläfenbein-MRT nach den Empfehlungen der Deutschen Röntgengesellschaft erhalten (mit T2*-Sequenz zur Diagnose einer superfiziellen Siderose) [116]. Eine beidseitige Kontrastmittelanreicherung im Kleinhirnbrückenwinkel wird ausser bei neoplastischen Prozessen auch bei infektiösen und autoimmunen Ursachen beobachtet. Ein HRCT oder DVT des Schläfenbeins sollte insbesondere zur Diagnose einer Schädelbasisfraktur ( $\triangleright$ Abb. $\mathbf{3}$ ) oder einer Fehlbildung des Innenohres angefertigt werden [309].

- Bei der Labordiagnostik ( Tab. 10) sollte der Fokus zunächst auf behandelbaren Ursachen wie Vitamin B-/Folsäuremangel, Hypothyreose oder Diabetes liegen [360]. Infektiöse Ursachen sind selten. Da diese aber eine behandelbare Ursache darstellen, ist eine Bestimmung der Infektionsserologie neurotroper Bakterien und Viren durchaus gerechtfertigt. Wichtig ist in diesem Zusammenhang die Zusammenarbeit mit einem Infektiologen bei auffälligen Befunden [298].

- Die zusätzliche Bestimmung von Autoantikörpern (z. B. ANAs, ANCAs, Rheumafaktor) führte in einer retrospektiven Beobachtungsstudie an 154 BVP-Patienten nur in einem Fall zu einer therapeutischen Konsequenz (Therapie mit Corticosteroiden). Daher ist es empfehlenswert, eine umfassende Autoimmundiagnostik in Abhängigkeit von der Systemanamnese und der klinischen Präsentation in Abstimmung mit einem Immunologen zu planen [309].

- Liegen neben der BVP auch neurologische Symptome vor (Kapitel 4.1.3.2), so sollte der Patient neurologisch vorgestellt werden zur Planung der weiteren Diagnostik (z. B. Lumbalpunktion, Bestimmung antineuronaler Antikörper, elektrophysiologische Untersuchungen) und Therapie [360].

\subsubsection{Therapie}

Aktuell gibt es keine kausale Therapie der BVP. Daher ist es essentiell, Auslöser zu vermeiden, die ersten Symptome frühzeitig zu erkennen und - falls möglich - zugrundliegende Ursachen zu behandeln. Um eine weitere Verschlechterung der Vestibularisfunktion zu vermeiden, sollten der Patient und sein Hausarzt über potentiell vestibulotoxische Medikamente informiert werden, damit diese gemieden bzw. durch andere ersetzt werden können ( $\triangleright$ Tab. 9). Patienten mit BVP profitieren von einer gezielten vestibulären Rehabilitation zur Förderung der zentral-vestibulären Kompensation (bei noch vorhandener vestibulärer Restfunktion) und der somatosensorischen Substitution (Übernahme verloren gegangener Funktion durch das visuelle und somatosensorische System) [67, 361, 362].

Im Rahmen von klinischen Studien werden aktuell somatosensorische Assistenzsysteme (z. B. vibrotaktiles Feedback) oder die transmastoidale Stimulation mit galvanischem «Noise» (Verbesserung der Detektion vestibulärer Reize durch das Prinzip der stochastischen Resonanz bei vorhandener peripher-vestibulärer Restfunktion) zur Verbesserung der Stand- und Gangsicherheit bei Patienten mit BVP erprobt [62, 363, 364].

Eine Wiederherstellung verloren gegangener peripher-vestibulärer Funktion ist nach aktuellem Stand der Forschung nur durch ein vestibuläres Implantat möglich. In Analogie zum Cochlea-Implantat werden hier Drehbeschleunigungen des Kopfes durch ein extern am Kopf getragenes Gyroskop detektiert und vom Implantat in ein elektrisches Signal umgewandelt, welches über Elektroden die entsprechenden Äste des N. vestibularis stimuliert [365370].

\section{Danksagung}

Ich danke Dr. D. Bächinger, Dr. F. Fierz, Prof. A. Huber, Dr. E. Probst, Prof. D. Straumann, PD Dr. K. Weber (Universitätsspital Zürich), Prof. I.S. Curthoys, Prof. G.M. Halmagyi (University of Sydney) und Prof. S.K. Plontke (Universitätsklinikum Halle/Saale) für wertvolle Anregungen und Diskussionen bei der Erstellung des Manuskriptes. Mein besonderer Dank gilt Dr. S. Siebert für die professionelle Unterstützung bei der Erstellung der Abbildungen und die kritische Durchsicht des Manuskriptes. 


\section{Interessenkonflikt}

Prof. Dlugaiczyk wurden Teilnahmegebühren für Kongresse, Reise- und Übernachtungskosten erstattet von Hennig Arzneimittel und Otometrics. Für Vorträge wurde sie honoriert von Otometrics und Ewopharma. Für die Durchführung von klinischen Studien erhielt sie Gelder von Otonomy.

\section{Literatur}

[1] Ehlers-Danlos Support UK. "Why the zebra?" Online: https://www. ehlers-danlos.org/about-us/charity-aims-and-focus/why-thezebra/\#: : :text = Ehlers \%2DDanlos \%20syndrome \%20is \%20 considered,help \%20bring \%20our \%20community \%20together; abgerufen am 04.12.2020

[2] Neuhauser HK. The epidemiology of dizziness and vertigo. Handb Clin Neurol 2016; 137: 67-82

[3] Brandt T, Strupp M, Dieterich M. Vestibular paroxysmia: a treatable neurovascular cross-compression syndrome. J Neurol 2016; 263 : S90-96

[4] Orphanet. "Das Portal für seltene Krankheiten und Orphan Drugs" Online: https://www.orpha.net/consor/cgi-bin/index.php; abgerufen am 24.10.2020

[5] Bisdorff A. The Bárány Classification of vestibular disorders, its clinical implementation and future prospects. HNO 2020; 68: 304-312

[6] Bárány Society. "International Classification of Vestibular Disorders (ICVD)". Online: http://www.jvr-web.org/ICVD.html; abgerufen am 24.10.2020

[7] Halmagyi GM, Chen L, MacDougall HG et al. The Video Head Impulse Test. Front Neurol 2017; 8: 258

[8] Rosengren SM, Colebatch JG, Young AS et al. Vestibular evoked myogenic potentials in practice: Methods, pitfalls and clinical applications. Clin Neurophysiol Pract 2019; 4: 47-68

[9] Papathanasiou ES, Straumann D. Why and when to refer patients for vestibular evoked myogenic potentials: A critical review. Clin Neurophysiol 2019; 130: 1539-1556

[10] Dlugaiczyk J. Evidence-based diagnostic use of VEMPs : From neurophysiological principles to clinical application. German version. HNO 2020; 68: 324-335

[11] Curthoys IS, Dlugaiczyk J. Physiology, clinical evidence and diagnostic relevance of sound-induced and vibration-induced vestibular stimulation. Curr Opin Neurol 2020; 33: 126-135

[12] Dlugaiczyk J. "Functional Aspects of Vestibular Evoked Myogenic Potentials". In: Fritzsch B, Straka, H, Hrsg. "The Senses: A Comprehensive Reference”. 2. Aufl. Amsterdam: Elsevier; 2020: 672-698

[13] Curthoys IS. The interpretation of clinical tests of peripheral vestibular function. Laryngoscope 2012; 122: 1342-1352

[14] Walther LE. Current Diagnostic Procedures for Diagnosing Vertigo and Dizziness. Laryngorhinootologie 2017; 96: S183-S208

[15] Schmäl F. Effective diagnostics for vertigo, dizziness and equilibrium disorders. HNO 2020; 68: 703-716

[16] Curthoys IS, Grant JW, Burgess AM et al. Otolithic Receptor Mechanisms for Vestibular-Evoked Myogenic Potentials: A Review. Front Neurol 2018; 9: 366

[17] Voit M. Zur Frage der Verästelung des Nervus acusticus bei den Säugetieren. Anat Anz 1907; 31: 635-640

[18] Curthoys IS, Vulovic V, Burgess AM et al. Neural basis of new clinical vestibular tests: otolithic neural responses to sound and vibration. Clin Exp Pharmacol Physiol 2014; 41: 371-380
[19] Newman-Toker DE, Saber Tehrani AS, Mantokoudis G et al. Quantitative video-oculography to help diagnose stroke in acute vertigo and dizziness: toward an ECG for the eyes. Stroke 2013; 44: $1158-1161$

[20] MacDougall HG, Holden J, Rosengren SM et al. VEMP: A Portable Interface to Record Vestibular Evoked Myogenic Potentials (VEMPs) With a Smart Phone or Tablet. Front Neurol 2018; 9: 543

[21] Young AS, Lechner C, Bradshaw AP et al. Capturing acute vertigo: A vestibular event monitor. Neurology 2019; 92: e2743-e2753

[22] Shaikh AG, Bronstein A, Carmona S et al. Consensus on Virtual Management of Vestibular Disorders: Urgent Vs. Expedited Care. Cerebellum. 2020; DOI: 10.1007/s12311-020-01178-8: 1-5

[23] Kösling S. Modern imaging of the temporal bone. HNO 2017; 65: 462-471

[24] Pyykkö I, Zou J, Gürkov R et al. Imaging of Temporal Bone. Adv Otorhinolaryngol 2019; 82: 12-31

[25] Navi BB, Kamel H, Shah MP et al. Application of the ABCD2 score to identify cerebrovascular causes of dizziness in the emergency department. Stroke 2012; 43: 1484-1489

[26] Kattah JC, Talkad AV, Wang DZ et al. HINTS to diagnose stroke in the acute vestibular syndrome: three-step bedside oculomotor examination more sensitive than early MRI diffusion-weighted imaging. Stroke 2009; 40: 3504-3510

[27] Newman-Toker DE, Kerber KA, Hsieh YH et al. HINTS outperforms $A B C D 2$ to screen for stroke in acute continuous vertigo and dizziness. Acad Emerg Med 2013; 20: 986-996

[28] Saber Tehrani AS, Kattah JC, Kerber KA et al. Diagnosing Stroke in Acute Dizziness and Vertigo: Pitfalls and Pearls. Stroke 2018; 49: 788-795

[29] Kattah JC. Use of HINTS in the acute vestibular syndrome. An Overview. Stroke Vasc Neurol 2018; 3: 190-196

[30] Tarnutzer AA, Berkowitz AL, Robinson KA et al. Does my dizzy patient have a stroke? A systematic review of bedside diagnosis in acute vestibular syndrome. CMAJ 2011; 183: E571-E592

[31] Newman-Toker DE, Curthoys IS, Halmagyi GM. Diagnosing Stroke in Acute Vertigo: The HINTS Family of Eye Movement Tests and the Future of the "Eye ECG". Semin Neurol 2015; 35: 506-521

[32] Newman-Toker DE, Edlow JA. TiTrATE: A Novel, Evidence-Based Approach to Diagnosing Acute Dizziness and Vertigo. Neurol Clin 2015; 33: 577-599. viii

[33] Bisdorff A, Von Brevern M, Lempert T et al. Classification of vestibular symptoms: towards an international classification of vestibular disorders. J Vestib Res 2009; 19: 1-13

[34] Fetter M, Dichgans J. Vestibular neuritis spares the inferior division of the vestibular nerve. Brain 1996; 119: 755-763

[35] Taylor RL, McGarvie LA, Reid N et al. Vestibular neuritis affects both superior and inferior vestibular nerves. Neurology 2016; 87: 1704-1712

[36] Aw ST, Fetter M, Cremer PD et al. Individual semicircular canal function in superior and inferior vestibular neuritis. Neurology 2001; 57: 768-774

[37] Halmagyi GM, Aw ST, Karlberg M et al. Inferior vestibular neuritis. Ann N Y Acad Sci 2002; 956: 306-313

[38] Monstad P, Okstad S, Mygland A. Inferior vestibular neuritis: 3 cases with clinical features of acute vestibular neuritis, normal calorics but indications of saccular failure. BMC Neurol 2006; 6: 45

[39] Zhang D, Fan Z, Han Y et al. Inferior vestibular neuritis: a novel subtype of vestibular neuritis. J Laryngol Otol 2010; 124: 477-481

[40] Kim JS, Kim HJ. Inferior vestibular neuritis. J Neurol 2012; 259: 1553-1560 
[41] Walther LE, Repik I. Inferior vestibular neuritis: diagnosis using VEMP. HNO 2012; 60: 126-131

[42] Jeong SH, Kim HJ, Kim JS. Vestibular neuritis. Semin Neurol 2013; 33 : 185-194

[43] Magliulo G, Gagliardi S, Ciniglio Appiani M et al. Selective vestibular neurolabyrinthitis of the lateral and superior semicircular canal ampulla and ampullary nerves. Ann Otol Rhinol Laryngol 2012; 121: 640-644

[44] Goebel JA, O'Mara W, Gianoli G. Anatomic considerations in vestibular neuritis. Otol Neurotol 2001; 22: 512-518

[45] Gianoli G, Goebel J, Mowry S et al. Anatomic differences in the lateral vestibular nerve channels and their implications in vestibular neuritis. Otol Neurotol 2005; 26: 489-494

[46] Ewald JR. "Physiologische Untersuchungen über das Endorgan des Nervus octavus". Wiesbaden: J.F. Bergmann; 1892

[47] Cohen B, Suzuki JI, Bender MB. Eye Movements from Semicircular Canal Nerve Stimulation in the Cat. Ann Otol Rhinol Laryngol 1964; 73: 153-169

[48] Cremer PD, Migliaccio AA, Pohl DV et al. Posterior semicircular canal nystagmus is conjugate and its axis is parallel to that of the canal. Neurology 2000; 54: 2016-2020

[49] Teixido M, Traboulsi H, Osier P et al. "BPPV viewer: Ewald's law in BPPV”. Online: https://bppvviewer.com/bppv-videos/ abgerufen am 10.10.2020

[50] Argaet EC, Bradshaw AP, Welgampola MS. Benign positional vertigo, its diagnosis, treatment and mimics. Clin Neurophysiol Pract 2019; 4: 97-111

[51] Manzari L, Burgess AM, Curthoys IS. Ocular and cervical vestibular evoked myogenic potentials in response to bone-conducted vibration in patients with probable inferior vestibular neuritis. J Laryngol Otol 2012; 126: 683-691

[52] Curthoys IS, Burgess AM, Manzari L. The Evidence for Selective Loss of Otolithic Function. Semin Neurol 2020; 40: 33-39

[53] Curthoys IS, Manzari L. Otolithic disease: clinical features and the role of vestibular evoked myogenic potentials. Semin Neurol 2013; 33 : 231-237

[54] Pelosi S, Schuster D, Jacobson GP et al. Clinical characteristics associated with isolated unilateral utricular dysfunction. Am J Otolaryngol 2013; 34: 490-495

[55] Fujimoto C, Suzuki S, Kinoshita M et al. Clinical features of otolith organ-specific vestibular dysfunction. Clin Neurophysiol 2018; 129: 238-245

[56] Manzari L, Burgess AM, Curthoys IS. Does unilateral utricular dysfunction cause horizontal spontaneous nystagmus? Eur Arch Otorhinolaryngol 2012; 269: 2441-2445

[57] Manzari L, MacDougall HG, Burgess AM et al. Selective otolith dysfunctions objectively verified. J Vestib Res 2014; 24: 365-373

[58] Curthoys IS, Markham CH. Convergence of labyrinthine influences on units in the vestibular nuclei of the cat. I. Natural stimulation. Brain Res 1971; 35: 469-490

[59] Dickman JD, Angelaki DE. Vestibular convergence patterns in vestibular nuclei neurons of alert primates. J Neurophysiol 2002; 88: 3518-3533

[60] Straka H, Holler S, Goto F. Patterns of canal and otolith afferent input convergence in frog second-order vestibular neurons. J Neurophysiol 2002; 88: 2287-2301

[61] Uchino $Y$, Sasaki M, Sato $H$ et al. Otolith and canal integration on single vestibular neurons in cats. Exp Brain Res 2005; 164: 271-285

[62] Dlugaiczyk J, Gensberger KD, Straka H. Galvanic vestibular stimulation: from basic concepts to clinical applications. J Neurophysiol 2019; 121: $2237-2255$
[63] Baloh RW. Acute unilateral and bilateral vestibular loss. Continuum (Minneap Minn) 2006; 12: 46-64

[64] Akin FW, Murnane OD, Hall CD et al. Vestibular consequences of mild traumatic brain injury and blast exposure: a review. Brain Inj 2017; 31: 1188-1194

[65] Lee JD, Park MK, Lee BD et al. Otolith function in patients with head trauma. Eur Arch Otorhinolaryngol 2011; 268: 1427-1430

[66] Liao YH, Young YH. Inner Ear Damage by Firecracker Trauma. Audiol Neurootol 2018; 23: 116-121

[67] Hall CD, Herdman SJ, Whitney SL et al. Vestibular Rehabilitation for Peripheral Vestibular Hypofunction: An Evidence-Based Clinical Practice Guideline: FROM THE AMERICAN PHYSICAL THERAPY ASSOCIATION NEUROLOGY SECTION. J Neurol Phys Ther 2016; 40: 124-155

[68] Strupp M, Dlugaiczyk ], Ertl-Wagner BB et al. Vestibular Disorders. Dtsch Arztebl Int 2020; 117: 300-310

[69] Kim HA, Lee H. Recent Advances in Understanding Audiovestibular Loss of a Vascular Cause. J Stroke 2017; 19: 61-66

[70] Kim JS, Cho KH, Lee H. Isolated labyrinthine infarction as a harbinger of anterior inferior cerebellar artery territory infarction with normal diffusion-weighted brain MRI. J Neurol Sci 2009; 278: 82-84

[71] Kim JS, Lee H. Inner ear dysfunction due to vertebrobasilar ischemic stroke. Semin Neurol 2009; 29: 534-540

[72] Pogson JM, Taylor RL, Young AS et al. Vertigo with sudden hearing loss: audio-vestibular characteristics. J Neurol 2016; 263: 2086-2096

[73] Lee $\mathrm{H}$. Audiovestibular loss in anterior inferior cerebellar artery territory infarction: a window to early detection? J Neurol Sci 2012; 313: $153-159$

[74] Lee H, Kim JS, Chung EJ et al. Infarction in the territory of anterior inferior cerebellar artery: spectrum of audiovestibular loss. Stroke 2009; 40: 3745-3751

[75] Akdal G, Halmagyi G. Sudden vertigo in a 49-year-old man. J Clin Neurosci 2012; 19: 1751

[76] Liqun Z, Park KH, Kim H] et al. Acute Unilateral Audiovestibulopathy due to Embolic Labyrinthine Infarction. Front Neurol 2018; 9: 311

[77] Choi BY, An YH, Park JH et al. Audiological and surgical evidence for the presence of a third window effect for the conductive hearing loss in DFNX2 deafness irrespective of types of mutations. Eur Arch Otorhinolaryngol 2013; 270: 3057-3062

[78] Byun S, Lee JY, Kim BG et al. Acute vertigo and sensorineural hearing loss from infarction of the vestibulocochlear nerve: A case report. Medicine (Baltimore) 2018; 97: e12777

[79] Berrettini S, Seccia V, Fortunato $S$ et al. Analysis of the 3-dimensional fluid-attenuated inversion-recovery (3D-FLAIR) sequence in idiopathic sudden sensorineural hearing loss. JAMA Otolaryngol Head Neck Surg 2013; 139: 456-464

[80] Eliezer M, Toupet M, Guichard JP et al. Cochleovestibular artery syndrome: consideration based on VHIT, VEMP, and inner ear MRI. J Neurol 2019; 266: 2327-2329

[81] Eliezer M, Verillaud B, Guichard JP et al. Labyrinthine infarction caused by vertebral artery dissection: consideration based on MRI. J Neurol 2019; 266: 2575-2577

[82] Lee S], Lee SA, Kim BG et al. Feasibility of magnetic resonance imaging in the differential diagnosis of isolated acute audiovestibular loss. J Vestib Res 2018; 28: 385-391

[83] Zwergal A, Kirsch V, Gerb J et al. Neuro-otology: at the borders of ear and brain. Nervenarzt 2018; 89: 1106-1114

[84] Blum CA, Kasner SE. Transient Ischemic Attacks Presenting with Dizziness or Vertigo. Neurol Clin 2015; 33: 629-642. ix

[85] Schmiz A, Haibt-Lüttke G, Albrecht $G$ et al. Thrombosis of the basilar artery - a rare differential sudden deafness diagnosis and vestibular failure. Laryngorhinootologie 2000; 79: 253-259 
[86] Hennerici MG, Kern R. „Diagnostik akuter zerebrovaskulärer Erkrankungen, S1-Leitlinie, 2017“. In: Deutsche Gesellschaft für Neurologie, Hrsg. „Leitlinien für Diagnostik und Therapie in der Neurologie“. Online: https://dgn.org/leitlinien/030-117-diagnostik-akuterzerebrovaskulaerer-erkrankungen-2017/ abgerufen am: 11.10.2020

[87] Choi KD, Chun JU, Han MG et al. Embolic internal auditory artery infarction from vertebral artery dissection. J Neurol Sci 2006; 246: 169-172

[88] Marciniec M, Sapko K, Kulczyński M et al. Non-traumatic cervical artery dissection and ischemic stroke: A narrative review of recent research. Clin Neurol Neurosurg 2019; 187: 105561

[89] Ringelstein EB, Dittrich R. „Spontane Dissektion der extra- und intrakraniellen hirnversorgenden Arterien, S1-Leitlinie, 2016“. In: Deutsche Gesellschaft für Neurologie, Hrsg. „Leitlinien für Diagnostik und Therapie in der Neurologie“. Online: dgn.org/leitlinien/030005-spontane-dissektionen-der-extrakraniellen-und-intrakraniellenhirnversorgenden-arterien-2016/ abgerufen am 11.10.2020

[90] Pezier T, Barath K, Hegemann S. Partial Recovery of Audiological, Vestibular, and Radiological Findings following Spontaneous Intralabyrinthine Haemorrhage. Case Rep Otolaryngol 2013; 2013: 941530

[91] Araujo-Martins ], Melo P, Ribeiro C et al. Recovery of cochlear and vestibular function after labyrinthine haemorrhage. Acta Med Port 2014; 27: 649-651

[92] Wu X, Chen K, Sun L et al. Magnetic resonance imaging-detected inner ear hemorrhage as a potential cause of sudden sensorineural hearing loss. Am J Otolaryngol 2014; 35: 318-323

[93] Chern A, Famuyide AO, Moonis G et al. Bilateral Sudden Sensorineural Hearing Loss and Intralabyrinthine Hemorrhage in a Patient With COVID-19. Otol Neurotol 2021; 42: e10-e14

[94] Farshad-Amacker NA, Scheffel H, Frauenfelder T et al. Brainstem abnormalities and vestibular nerve enhancement in acute neuroborreliosis. BMC Res Notes 2013; 6: 551

[95] Nomiya S, Cureoglu S, Kariya S et al. Posterior semicircular canal dehiscence: a histopathologic human temporal bone study. Otol Neurotol 2010; 31: 1122-1127

[96] Malayala SV, Raza A. A Case of COVID-19-Induced Vestibular Neuritis. Cureus 2020; 12: e8918

[97] Zubair AS, McAlpine LS, Gardin T et al. Neuropathogenesis and Neurologic Manifestations of the Coronaviruses in the Age of Coronavirus Disease 2019: A Review. JAMA Neurol 2020; 77: 1018-1027

[98] Lamounier P, Franco Gonçalves V, Ramos HVL et al. A 67-Year-Old Woman with Sudden Hearing Loss Associated with SARS-CoV-2 Infection. Am J Case Rep 2020; 21: e927519

[99] Koumpa FS, Forde CT, Manjaly JG. Sudden irreversible hearing loss post COVID-19. BMJ Case Rep 2020; 13: e238419

[100] Almufarrij I, Uus K, Munro KJ. Does coronavirus affect the audiovestibular system? A rapid systematic review. Int J Audiol 2020; 59: 487-491

[101] Karimi-Galougahi M, Naeini AS, Raad N et al. Vertigo and hearing loss during the COVID-19 pandemic - is there an association? Acta Otorhinolaryngol Ital 2020; doi: 10.14639/0392-100x-n0820

[102] Maharaj S, Bello Alvarez M, Mungul S et al. Otologic dysfunction in patients with COVID-19: A systematic review. Laryngoscope Investig Otolaryngol 2020; 5: 1192-1196

[103] Young AS, Carroll AS, Welgampola MS et al. Acute unilateral peripheral vestibulopathy in neurosyphilis. J Neurol Sci 2017; 378: $55-58$

[104] Büttner U, Ott M, Helmchen C et al. Bilateral loss of eighth nerve function as the only clinical sign of vertebrobasilar dolichoectasia. J Vestib Res 1995; 5: 47-51
[105] Yacovino DA, Finlay JB, Urbina Jaimes VN et al. Acute Bilateral Superior Branch Vestibular Neuropathy. Front Neurol 2018; 9: 353

[106] Ichijo K, Kinoshita M, Fujimoto C et al. Acute bilateral vestibulopathy with simultaneous involvement of both superior and inferior vestibular nerves. Auris Nasus Larynx 2020; 47: 905-908

[107] Petersen JA, Straumann D, Weber KP. Clinical diagnosis of bilateral vestibular loss: three simple bedside tests. Ther Adv Neurol Disord 2013; 6: 41-45

[108] Brandt T, Dieterich M. Vestibular paroxysmia: vascular compression of the eighth nerve? Lancet 1994; 343: 798-799

[109] Ryu H, Yamamoto S, Sugiyama K et al. Neurovascular compression syndrome of the eighth cranial nerve. Can the site of compression explain the symptoms? Acta Neurochir (Wien) 1999; 141: 495-501

[110] Hufner K, Barresi D, Glaser M et al. Vestibular paroxysmia: diagnostic features and medical treatment. Neurology 2008; 71: 1006-1014

[111] Donahue JH, Ornan DA, Mukherjee S. Imaging of Vascular Compression Syndromes. Radiol Clin North Am 2017; 55: 123-138

[112] Best C, Gawehn J, Krämer HH et al. MRI and neurophysiology in vestibular paroxysmia: contradiction and correlation. J Neurol Neurosurg Psychiatry 2013; 84: 1349-1356

[113] Guclu B, Sindou M, Meyronet D et al. Anatomical study of the central myelin portion and transitional zone of the vestibulocochlear nerve. Acta Neurochir (Wien) 2012; 154: 2277-2283. discussion 2283

[114] Urban PP, Bruning R. Vestibular paroxysmia and paroxysmal tinnitus. Nervenarzt 2018; 89: 204-206

[115] Bradley JP, Hullar TE, Neely JG et al. Hyperventilation-induced nystagmus and vertigo after stereotactic radiotherapy for vestibular schwannoma. Otol Neurotol 2011; 32: 1336-1338

[116] AG Kopf-Halsdiagnostik in der Deutschen Röntgengesellschaft. "Stellungnahmen und Empfehlungen". Online: https://www. ag-kopf-hals.drg.de/de-DE/295/stellungnahmen-und-empfehlungen/; abgerufen am 10.10.2020

[117] Arbusow V, Strupp M, Dieterich M et al. Alternating episodes of vestibular nerve excitation and failure. Neurology 1998; 51 : $1480-1483$

[118] Lee SU, Jeong SH, Kim HJ et al. Cerebellopontine angle meningioma mimicking vestibular paroxysmia. J Neurol 2016; 263: 168-170

[119] Young AS, Jonker B, Welgampola MS. Vestibular paroxysmia presenting with irritative nystagmus. Neurology 2019; 92: 723-724

[120] Han J, Wang T, Xie Y et al. Successive occurrence of vertebrobasilar dolichectasia induced trigeminal neuralgia, vestibular paroxysmia and hemifacial spasm: A case report. Medicine (Baltimore) 2018; 97: e11192

[121] Unkelbach MH, Radeloff A, Bink A et al. Megadolichobasilar anomaly causing acute deafness with vertigo. HNO 2008; 56: 62-64

[122] Bayer O, Bremova T, Strupp M et al. A randomized double-blind, placebo-controlled, cross-over trial (Vestparoxy) of the treatment of vestibular paroxysmia with oxcarbazepine. J Neurol 2018; 265 : 291-298

[123] Strupp M, Elger C, Goldschagg N. Treatment of vestibular paroxysmia with lacosamide. Neurol Clin Pract 2019; 9: 539-541

[124] Huang $\mathrm{CH}$, Young YH. Ocular and cervical vestibular-evoked myogenic potentials in Tumarkin falls. Otol Neurotol 2012; 33: $1251-1256$

[125] Wu Q, Li X, Sha Y et al. Clinical features and management of Meniere's disease patients with drop attacks. Eur Arch Otorhinolaryngol 2019; 276: 665-672

[126] Tumarkin A. THE OTOLITHIC CATASTROPHE: A NEW SYNDROME. Br Med J 1936; 2: 175-177

[127] Baloh RW, Jacobson K, Winder T. Drop attacks with Menière's syndrome. Ann Neurol 1990; 28: 384-387 
[128] Weiss NM. Seltene Erkrankungen des Mittelohres und der lateralen Schädelbasis. Laryngo-Rhino-Otol 2021; (accepted for publication)

[129] Ho ML, Moonis G, Halpin CF et al. Spectrum of Third Window Abnormalities: Semicircular Canal Dehiscence and Beyond. AJNR Am J Neuroradiol 2017; 38: 2-9

[130] Ho ML. Third Window Lesions. Neuroimaging Clin N Am 2019; 29 : 57-92

[131] Merchant SN, Rosowski J]. Conductive hearing loss caused by third-window lesions of the inner ear. Otol Neurotol 2008; 29: 282-289

[132] Pogson JM, Taylor RL, Thompson EO et al. A Window Into the Whole Story: Temporal Bone Plasmacytoma Presenting With a Mobile Third Window. Laryngoscope 2020; doi: 10.1002/lary.28951

[133] Rosowski JJ, Songer JE, Nakajima HH et al. Clinical, experimental, and theoretical investigations of the effect of superior semicircular canal dehiscence on hearing mechanisms. Otol Neurotol 2004; 25: 323-332

[134] Carey JP, Hirvonen TP, Hullar TE et al. Acoustic responses of vestibular afferents in a model of superior canal dehiscence. Otol Neurotol 2004; 25: 345-352

[135] Grieser B], Kleiser L, Obrist D. Identifying Mechanisms Behind the Tullio Phenomenon: a Computational Study Based on First Principles. J Assoc Res Otolaryngol 2016; 17: 103-118

[136] Iversen MM, Zhu H, Zhou W et al. Sound abnormally stimulates the vestibular system in canal dehiscence syndrome by generating pathological fluid-mechanical waves. Sci Rep 2018; 8: 10257

[137] Iversen MM, Rabbitt RD. Biomechanics of Third Window Syndrome. Front Neurol 2020; 11: 891

[138] Dlugaiczyk J, Burgess AM, Goonetilleke SC et al. Superior Canal Dehiscence Syndrome: Relating Clinical Findings With Vestibular Neural Responses From a Guinea Pig Model. Otol Neurotol 2019; 40: e406-e414

[139] Tullio P. “The Ear and the Genesis of Language and Writing”. Berlin, Wien: Urban \& Schwarzenberg; 1929

[140] Addams-Williams ], Wu K, Ray J. The experiments behind the Tullio phenomenon. J Laryngol Otol 2014; 128: 223-227

[141] Kaski D, Davies R, Luxon L et al. The Tullio phenomenon: a neurologically neglected presentation. J Neurol 2012; 259: 4-21

[142] Steenerson KK, Crane BT, Minor LB. Superior Semicircular Canal Dehiscence Syndrome. Semin Neurol 2020; 40: 151-159

[143] Minor LB, Solomon D, Zinreich JS et al. Sound- and/or pressure-induced vertigo due to bone dehiscence of the superior semicircular canal. Arch Otolaryngol Head Neck Surg 1998; 124: 249-258

[144] Tavassolie TS, Penninger RT, Zuñiga MG et al. Multislice computed tomography in the diagnosis of superior canal dehiscence: how much error, and how to minimize it? Otol Neurotol 2012; 33: 215-222

[145] Mittmann P, Ernst A, Seidl R et al. Superior Canal Dehiscence: A Comparative Postmortem Multislice Computed Tomography Study. OTO Open 2018; 2: 2473974x18793576

[146] Baxter M, McCorkle C, Trevino Guajardo C et al. Clinical and Physiologic Predictors and Postoperative Outcomes of Near Dehiscence Syndrome. Otol Neurotol 2019; 40: 204-212

[147] Re M, Gioacchini FM, Salvolini U et al. Multislice computed tomography overestimates superior semicircular canal dehiscence syndrome. Ann Otol Rhinol Laryngol 2013; 122: 625-631

[148] Carey JP, Minor LB, Nager GT. Dehiscence or thinning of bone overlying the superior semicircular canal in a temporal bone survey. Arch Otolaryngol Head Neck Surg 2000; 126: 137-147

[149] Watters KF, Rosowski J], Sauter T et al. Superior semicircular canal dehiscence presenting as postpartum vertigo. Otol Neurotol 2006; 27: 756-768
[150] Rajenderkumar D, Farrell KL, Alles RM et al. Multiple dehiscence of semicircular canals. J Laryngol Otol 2007; 121: 80-82

[151] Manzari L. Multiple dehiscences of bony labyrinthine capsule. A rare case report and review of the literature. Acta Otorhinolaryngol Ital 2010; 30: 317-320

[152] Manzari L, Modugno GC. Bilateral dehiscence of both superior and posterior semicircular canals. Otol Neurotol 2009; 30: 423-425

[153] Suryanarayanan R, Lesser TH. 'Honeycomb' tegmen: multiple tegmen defects associated with superior semicircular canal dehiscence. J Laryngol Otol 2010; 124: 560-563

[154] Westhofen M. Enlarged vestibular aqueduct syndrome - dehiscence syndromes - honeycomb mastoid: Pathophysiology and evidence for clinical differentiation. HNO 2020; 68: 336-343

[155] Ernst A, Todt I, Wagner J. Dehiscence syndromes: Diagnosis and treatment. HNO 2016; 64: 790-796

[156] Naert L, Van de Berg R, Van de Heyning P et al. Aggregating the symptoms of superior semicircular canal dehiscence syndrome. Laryngoscope 2018; 128: 1932-1938

[157] Hennebert C. A new syndrome in hereditary syphilis of the labyrinth. Press Med Belg Brux 1911; 63: 467

[158] Tilikete C, Krolak-Salmon P, Truy E et al. Pulse-synchronous eye oscillations revealing bone superior canal dehiscence. Ann Neurol 2004; 56: 556-560

[159] Young AS, McMonagle B, Pohl DV et al. Superior semicircular canal dehiscence presenting with recurrent positional vertigo. Neurology 2019; 93: 1070-1072

[160] Aw ST, Todd M], Aw GE et al. Click-evoked vestibulo-ocular reflex: stimulus-response properties in superior canal dehiscence. Neurology 2006; 66: 1079-1087

[161] Ward BK, Carey JP, Minor LB. Superior Canal Dehiscence Syndrome: Lessons from the First 20 Years. Front Neurol 2017; 8: 177

[162] Dumas G, Curthoys IS, Lion A et al. The Skull Vibration-Induced Nystagmus Test of Vestibular Function-A Review. Front Neurol 2017; 8: 41

[163] Dumas G, Tan H, Dumas L et al. Skull vibration induced nystagmus in patients with superior semicircular canal dehiscence. Eur Ann Otorhinolaryngol Head Neck Dis 2019; 136: 263-272

[164] Dlugaiczyk J, Burgess AM, Curthoys IS. Activation of Guinea Pig Irregular Semicircular Canal Afferents by $100 \mathrm{~Hz}$ Vibration: Clinical Implications for Vibration-induced Nystagmus and Vestibular-evoked Myogenic Potentials. Otol Neurotol 2020; 41: e961-e970

[165] Brantberg K, Verrecchia L, Westin M. Enhanced Auditory Sensitivity to Body Vibrations in Superior Canal Dehiscence Syndrome. Audiol Neurootol 2016; 21: 365-371

[166] Gürkov R, Jerin C, Flatz W et al. Superior canal dehiscence syndrome : Diagnosis with vestibular evoked myogenic potentials and fremitus nystagmus. German version. HNO 2018; 66: 390-395

[167] Zuniga MG, Janky KL, Nguyen KD et al. Ocular vs. cervical VEMPs in the diagnosis of superior semicircular canal dehiscence syndrome. Otol Neurotol 2013; 34: 121-126

[168] Janky KL, Nguyen KD, Welgampola M et al. Air-conducted oVEMPs provide the best separation between intact and superior canal dehiscent labyrinths. Otol Neurotol 2013; 34: 127-134

[169] Manzari L, Burgess AM, McGarvie LA et al. Ocular and cervical vestibular evoked myogenic potentials to $500 \mathrm{~Hz} f z$ bone-conducted vibration in superior semicircular canal dehiscence. Ear Hear 2012; 33: 508-520

[170] Verrecchia L, Brantberg K, Tawfique Z et al. Diagnostic Accuracy of Ocular Vestibular Evoked Myogenic Potentials for Superior Canal Dehiscence Syndrome in a Large Cohort of Dizzy Patients. Ear Hear 2019; 40: 287-294 
[171] Manzari L, Burgess AM, McGarvie LA et al. An indicator of probable semicircular canal dehiscence: ocular vestibular evoked myogenic potentials to high frequencies. Otolaryngol Head Neck Surg 2013; 149: $142-145$

[172] Tran ED, Swanson A, Sharon JD et al. Ocular Vestibular-Evoked Myogenic Potential Amplitudes Elicited at $4 \mathrm{kHz}$ Optimize Detection of Superior Semicircular Canal Dehiscence. Front Neurol 2020; 11: 879

[173] Curthoys IS, Burgess AM, Goonetilleke SC. Phase-locking of irregular guinea pig primary vestibular afferents to high frequency $(>250 \mathrm{~Hz})$ sound and vibration. Hear Res 2019; 373: 59-70

[174] Fröhlich L, Curthoys IS, Kösling S et al. Cervical and Ocular VestibularEvoked Myogenic Potentials in Patients With Intracochlear Schwannomas. Front Neurol 2020; 11: 549817

[175] Wenzel A, Ward BK, Ritzl EK et al. Intraoperative neuromonitoring for superior semicircular canal dehiscence and hearing outcomes. Otol Neurotol 2015; 36: 139-145

[176] Ward BK, Wenzel A, Ritzl EK et al. Electrocochleography summating potential seen on auditory brainstem response in a case of superior semicircular canal dehiscence. Surg Neurol Int 2017; 8: 90

[177] Weinreich HM, Carey JP. Perilymphatic Fistulas and Superior Semi-Circular Canal Dehiscence Syndrome. Adv Otorhinolaryngol 2019; 82: 93-100

[178] Bremke M, Luers JC, Anagiotos A et al. Comparison of digital volume tomography and high-resolution computed tomography in detecting superior semicircular canal dehiscence - a temporal bone study. Acta Otolaryngol 2015; 135: 901-906

[179] Browaeys P, Larson TL, Wong ML et al. Can MRI replace CT in evaluating semicircular canal dehiscence? AJNR Am J Neuroradiol 2013; 34: 1421-1427

[180] Ward BK, Wenzel A, Ritzl EK et al. Near-dehiscence: clinical findings in patients with thin bone over the superior semicircular canal. Otol Neurotol 2013; 34: 1421-1428

[181] Mehta R, Klumpp ML, Spear SA et al. Subjective and objective findings in patients with true dehiscence vs. thin bone over the superior semicircular canal. Otol Neurotol 2015; 36: 289-294

[182] Spasic M, Trang A, Chung LK et al. Clinical Characteristics of Posterior and Lateral Semicircular Canal Dehiscence. J Neurol Surg B Skull Base 2015; 76: 421-425

[183] Lee JA, Liu YF, Nguyen SA et al. Posterior Semicircular Canal Dehiscence: Case Series and Systematic Review. Otol Neurotol 2020; 41: $511-521$

[184] Hourani R, Carey J, Yousem DM. Dehiscence of the jugular bulb and vestibular aqueduct: findings on 200 consecutive temporal bone computed tomography scans. J Comput Assist Tomogr 2005; 29: 657-662

[185] Gopen Q, Zhou G, Poe D et al. Posterior semicircular canal dehiscence: first reported case series. Otol Neurotol 2010; 31: 339-344

[186] Friedmann DR, Eubig J, Winata LS et al. Prevalence of jugular bulb abnormalities and resultant inner ear dehiscence: a histopathologic and radiologic study. Otolaryngol Head Neck Surg 2012; 147: 750-756

[187] Friedmann DR, Eubig J, Winata LS et al. A clinical and histopathologic study of jugular bulb abnormalities. Arch Otolaryngol Head Neck Surg 2012; 138: 66-71

[188] Park JJ, Shen A, Loberg C et al. The relationship between jugular bulb position and jugular bulb related inner ear dehiscence: a retrospective analysis. Am J Otolaryngol 2015; 36: 347-351

[189] Helmchen C, Gehrking E, Gottschalk S et al. Persistence of perilymph fistula mechanism in a completely paretic posterior semicircular canal. J Neurol Neurosurg Psychiatry 2005; 76: 280-282
[190] Aw ST, Welgampola MS, Bradshaw AP et al. Click-evoked vestibuloocular reflex distinguishes posterior from superior canal dehiscence. Neurology 2010; 75: 933-935

[191] Bear ZW, McEvoy TP, Mikulec AA. Quantification of hearing loss in patients with posterior semicircular canal dehiscence. Acta Otolaryngol 2015; 135: 974-977

[192] Mikulec AA, Poe DS. Operative management of a posterior semicircular canal dehiscence. Laryngoscope 2006; 116: 375-378

[193] Lim HW, Park HJ, Jung JH et al. Surgical treatment of posterior semicircular canal dehiscence syndrome caused by jugular diverticulum. J Laryngol Otol 2012; 126: 928-931

[194] Philip A, Mammen MD, Lepcha A et al. Posterior semicircular canal dehiscence: a diagnostic and surgical conundrum. BMJ Case Rep. 2019; 12

[195] Zhang YB, Dai CF, Sha Y. Sound-induced vertigo due to bone dehiscence of the lateral semicircular canal. Eur Arch Otorhinolaryngol 2010; 267: 1319-1321

[196] Valvassori GE, Clemis JD. The large vestibular aqueduct syndrome. Laryngoscope 1978; 88: 723-728

[197] Campbell AP, Adunka OF, Zhou B et al. Large vestibular aqueduct syndrome: anatomic and functional parameters. Laryngoscope 2011; 121: $352-357$

[198] Vijayasekaran S, Halsted M], Boston M et al. When is the vestibular aqueduct enlarged? A statistical analysis of the normative distribution of vestibular aqueduct size. AJNR Am J Neuroradiol 2007; 28: 1133-1138

[199] Connor SE], Dudau C, Pai I et al. Is CT or MRI the optimal imaging investigation for the diagnosis of large vestibular aqueduct syndrome and large endolymphatic sac anomaly? Eur Arch Otorhinolaryngol 2019; 276: 693-702

[200] Everett LA, Glaser B, Beck JC et al. Pendred syndrome is caused by mutations in a putative sulphate transporter gene (PDS). Nat Genet 1997; 17: 411-422

[201] Smith RJH, Iwasa Y, Schaefer AM "Pendred Syndrome/Nonsyndromic Enlarged Vestibular Aqueduct”. In: Adam MP, Ardinger HH, Pagon RA et al, Hrsg. "GeneReviews(®)". Seattle (WA): University of Washington, Seattle; 1993-2020

[202] Wémeau JL, Kopp P. Pendred syndrome. Best Pract Res Clin Endocrinol Metab 2017; 31: 213-224

[203] Eckhard AH, Bächinger D, Nadol JB Jr. Absence of Endolymphatic Sac Ion Transport Proteins in Large Vestibular Aqueduct Syndrome - A Human Temporal Bone Study. Otol Neurotol 2020; 41: e1256e1263. doi: $10.1097 / \mathrm{mao} .0000000000002832$

[204] Merchant SN, Nakajima HH, Halpin C et al. Clinical investigation and mechanism of air-bone gaps in large vestibular aqueduct syndrome. Ann Otol Rhinol Laryngol 2007; 116: 532-541

[205] Manzari L. Vestibular signs and symptoms of volumetric abnormalities of the vestibular aqueduct. J Laryngol Otol 2008; 122: 557-563

[206] Gopen Q, Zhou G, Whittemore K et al. Enlarged vestibular aqueduct: review of controversial aspects. Laryngoscope 2011; 121: 1971-1978

[207] White J, Krakovitz P. Nystagmus in Enlarged Vestibular Aqueduct: A Case Series. Audiol Res 2015; 5: 120

[208] Brodsky JR, Choi SS. Should children with an enlarged vestibular aqueduct be restricted from playing contact sports? Laryngoscope 2018; 128: 2219-2220

[209] Alemi AS, Chan DK. Progressive Hearing Loss and Head Trauma in Enlarged Vestibular Aqueduct: A Systematic Review and Meta-analysis. Otolaryngol Head Neck Surg 2015; 153: 512-517

[210] Oh AK, Ishiyama A, Baloh RW. Vertigo and the enlarged vestibular aqueduct syndrome. J Neurol 2001; 248: 971-974 
[211] Song J], Hong SK, Kim JS et al. Enlarged vestibular aqueduct may precipitate benign paroxysmal positional vertigo in children. Acta Otolaryngol 2012; 132 (Suppl 1): S109-S117

[212] Sheykholeslami K, Schmerber S, Habiby Kermany M et al. Vestibularevoked myogenic potentials in three patients with large vestibular aqueduct. Hear Res 2004; 190: 161-168

[213] Zhou G, Gopen Q. Characteristics of vestibular evoked myogenic potentials in children with enlarged vestibular aqueduct. Laryngoscope 2011; 121: 220-225

[214] Taylor RL, Bradshaw AP, Magnussen JS et al. Augmented ocular vestibular evoked myogenic potentials to air-conducted sound in large vestibular aqueduct syndrome. Ear Hear 2012; 33: 768-771

[215] Manzari L. Enlarged vestibular aqueduct (EVA) related with recurrent benign paroxysmal positional vertigo (BPPV). Med Hypotheses 2008; 70: 61-65

[216] Song JJ, Hong SK, Lee SY et al. Vestibular Manifestations in Subjects With Enlarged Vestibular Aqueduct. Otol Neurotol 2018; 39: e461-e467

[217] Valvassori GE, Naunton RF, Lindsay JR. Inner ear anomalies: clinical and histopathological considerations. Ann Otol Rhinol Laryngol 1969; 78: 929-938

[218] Sugiura M, Sato E, Nakashima T et al. Long-term follow-up in patients with Pendred syndrome: vestibular, auditory and other phenotypes. Eur Arch Otorhinolaryngol 2005; 262: 737-743

[219] Luxon LM, Cohen M, Coffey RA et al. Neuro-otological findings in Pendred syndrome. Int J Audiol 2003; 42: 82-88

[220] Sone M, Yoshida T, Morimoto K et al. Endolymphatic hydrops in superior canal dehiscence and large vestibular aqueduct syndromes. Laryngoscope 2016; 126: 1446-1450

[221] McGarvie LA, Curthoys IS, MacDougall HG et al. What does the dissociation between the results of video head impulse vs. caloric testing reveal about the vestibular dysfunction in Ménière's disease? Acta Otolaryngol 2015; 135: 859-865

[222] Jung J, Suh M], Kim SH. Discrepancies between video head impulse and caloric tests in patients with enlarged vestibular aqueduct. Laryngoscope 2017; 127: 921-926

[223] Marangos N. Dysplasia of the inner ear and inner ear canal. HNO 2002; 50: 866-880. quiz 880-861

[224] McKusick-Nathans Institute of Genetic Medicine. "OMIM - Online Mendelian Inheritance in Man” Online: https://omim.org/ abgerufen am 10.10.2020

[225] Pendred V. Deaf-mutism and goitre. The Lancet 1896; 148: 532

[226] Tsukamoto K, Suzuki H, Harada D et al. Distribution and frequencies of PDS (SLC26A4) mutations in Pendred syndrome and nonsyndromic hearing loss associated with enlarged vestibular aqueduct: a unique spectrum of mutations in Japanese. Eur J Hum Genet 2003; 11: $916-922$

[227] Mondini C, Hartley G], Phelps PD. Minor Works of Carlo Mondini: The Anatomical Section of a Boy Born Deaf (Opuscula Caroli Mundini: Anatomica Surdi Nati Sectio. Carolus Mundinus). Am J Otol 1997; 18 : 288-293

[228] Wenzel A, Ward BK, Schubert MC et al. Patients with vestibular loss, tullio phenomenon, and pressure-induced nystagmus: vestibular atelectasis? Otol Neurotol 2014; 35: 866-872

[229] Finn S, Dietzek M, Karvouniari P et al. Bilateral vestibulopathy with positive Tullio phenomenon. Laryngoscope 2018; 128: 1223-1225

[230] Maslovara S, Butkovic-Soldo S, Pajic-Matic I et al. Vestibular atelectasis: Decoding pressure and sound-induced nystagmus with bilateral vestibulopathy. Laryngoscope 2019; 129: 1685-1688

[231] Kaski D, Bronstein AM. Patients with Vestibular Loss, Tullio Phenomenon, and Pressure-induced Nystagmus: Vestibular Atelectasis? Otol Neurotol 2016; 37: 115-116
[232] Merchant SN, Schuknecht HF. Vestibular atelectasis. Ann Otol Rhinol Laryngol 1988; 97: 565-576

[233] Eliezer M, Toupet M, Vitaux H et al. MRI Evidence of Vestibular Atelectasis in Bilateral Vestibulopathy and Tullio Phenomenon. Otol Neurotol 2019; 40: e944-e946

[234] Eliezer M, Attyé A, Guichard JP et al. Vestibular atelectasis: Myth or reality? Laryngoscope 2019; 129: 1689-1695

[235] Marc M, Hautefort C, Guichard JP et al. Clinical characteristics in unilateral vestibular atelectasis. J Neurol 2020; doi: 10.1007/ s00415-020-10220-y

[236] Lesinski A, Kempf HG, Lenarz T. Tullio phenomenon after cochlear implantation. HNO 1998; 46: 692-694

[237] Schick B, Dlugaiczyk J. Complications and pitfalls in surgery of the ear/lateral skull base. Laryngorhinootologie 2013; 92: S137-176

[238] Kacker SK, Hinchcliffe R. Unusual Tullio phenomena. J Laryngol Otol 1970; 84: 155-166

[239] Nadol JB Jr. Positive Hennebert's sign in Meniere's disease. Arch Otolaryngol 1977; 103: 524-530

[240] Suzuki M, Kitajima N, Ushio M et al. Changes in the Tullio phenomenon and the fistula sign in the course of endolymphatic hydrops. ORL J Otorhinolaryngol Relat Spec 2003; 65: 125-128

[241] von Brevern M, Bertholon P, Brandt T et al. Benign paroxysmal positional vertigo: Diagnostic criteria. J Vestib Res 2015; 25: 105-117

[242] Dlugaiczyk J, Siebert S, Hecker DJ et al. Involvement of the anterior semicircular canal in posttraumatic benign paroxysmal positioning vertigo. Otol Neurotol 2011; 32: 1285-1290

[243] Anagnostou E, Kouzi I. Spengos K. Diagnosis and Treatment of Anterior-Canal Benign Paroxysmal Positional Vertigo: A Systematic Review. J Clin Neurol 2015; 11: 262-267

[244] Teixido M, Traboulsi H, Osier P et al. "BPPV viewer: eye movement videos”. Online: https://bppvviewer.com/eye-movement-videos/ abgerufen am 10.10.2020

[245] Buki B, Simon L, Garab S et al. Sitting-up vertigo and trunk retropulsion in patients with benign positional vertigo but without positional nystagmus. J Neurol Neurosurg Psychiatry 2011; 82: 98-104

[246] Halmagyi GM. A new type of positional vertigo. J Neurol Neurosurg Psychiatry 2011; 82: 3

[247] Büttner U, Helmchen C, Brandt T. Diagnostic criteria for central vs. peripheral positioning nystagmus and vertigo: a review. Acta Otolaryngol 1999; 119: 1-5

[248] Van Abel KM, Carlson ML, Link M] et al. Primary inner ear schwannomas: a case series and systematic review of the literature. Laryngoscope 2013; 123: 1957-1966

[249] Frisch CD, Eckel LJ, Lane Jl et al. Intralabyrinthine schwannomas. Otolaryngol Clin North Am 2015; 48: 423-441

[250] Bagattini M, Quesnel AM, Röösli C. Histopathologic Evaluation of Intralabyrinthine Schwannoma. Audiol Neurootol 2020; doi: $10.1159 / 0005116341-8$

[251] Meyer O. Ein Fall von multiplen Tumoren in den Endausbreitungen des Akustikus. Z Ohrenheilk 1917; 75: 95-113

[252] Plontke SK, Rahne T, Pfister M et al. Intralabyrinthine schwannomas: Surgical management and hearing rehabilitation with cochlear implants. HNO 2017; 65: 136-148

[253] Marinelli JP, Lohse CM, Carlson ML. Incidence of Intralabyrinthine Schwannoma: A Population-based Study Within the United States. Otol Neurotol 2018; 39: 1191-1194

[254] Schulz C, Esser D, Rosahl S et al. Management of vestibular schwannomas. Laryngorhinootologie 2018; 97: 875-896 
[255] Choudhury B, Carlson ML, Jethanamest D. Intralabyrinthine Schwannomas: Disease Presentation, Tumor Management, and Hearing Rehabilitation. J Neurol Surg B Skull Base 2019; 80: 196-202

[256] Kennedy RJ, Shelton C, Salzman KL et al. Intralabyrinthine schwannomas: diagnosis, management, and a new classification system. Otol Neurotol 2004; 25: 160-167

[257] Salzman KL, Childs AM, Davidson HC et al. Intralabyrinthine schwannomas: imaging diagnosis and classification. AJNR Am J Neuroradiol 2012; 33: 104-109

[258] Jia H, Nguyen Y, Hochet B et al. NF2-Related Intravestibular Schwannomas: Long-Term Outcomes of Cochlear Implantation. Otol Neurotol 2020; 41: 94-99

[259] Withers S, Plontke SK, Boeddinghaus R et al. Bilateral intracochlear schwannomas in a patient with no genetic or clinical features of neurofibromatosis type 2. HNO 2020; 68: 60-64

[260] Jerin C, Krause E, Ertl-Wagner B et al. Clinical features of delayed endolymphatic hydrops and intralabyrinthine schwannoma : An imaging-confirmed comparative case series. German version. HNO 2016; 64: 911-916

[261] Venkatasamy A, Bretz P, Karol A et al. MRI of endolymphatic hydrops in patients with intralabyrinthine schwannomas: a case-controlled study using non-enhanced T2-weighted images at 3 T. Eur Arch Otorhinolaryngol 2020; 26. DOI: 10.1007/s00405-020-06271-6

[262] Plontke SK, Caye-Thomasen P, Strauss C et al. Management of transmodiolar and transmacular cochleovestibular schwannomas with and without cochlear implantation. German version. HNO 2020; 68: 734-748

[263] Plontke SK, Kosling S, Pazaitis N et al. Intracochlear schwannoma: Tumor removal via subtotal cochleoectomy and partial cochlear reconstruction with preservation of semicircular canal function. HNO 2017; 65: 158-162

[264] Vernooij MW, Ikram MA, Vincent AJ et al. Intravestibular lipoma: an important imaging diagnosis. Arch Otolaryngol Head Neck Surg 2008; 134: 1225-1228

[265] Choi JS, Kim YH, Han CD et al. Intravestibular space occupying lesions of lipoma and schwannoma. Auris Nasus Larynx 2012; 39: 431-433

[266] Aschendorff A, Arndt S, Laszig R et al. Treatment and auditory rehabilitation of intralabyrinthine schwannoma by means of cochlear implants - German Version. HNO 2017; 65: 321-327

[267] Plontke SK, Frohlich L, Wagner L et al. How Much Cochlea Do You Need for Cochlear Implantation? Otol Neurotol 2020; 41: 694-703

[268] Wagner L, Plontke S, Fröhlich L, Rahne T. Reduced spread of electric field after surgical removal of intracochlear schwannoma and cochlear implantation. Otol Neurotol 2020; 41: e1297-e1303

[269] Massager N, Drogba L, Delbrouck C et al. Gamma knife radiosurgery for intralabyrinthine schwannomas. J Radiosurg SBRT 2011; 1: 237-245

[270] Tuleasca C, George M, Schiappacasse L et al. Gamma Knife radiosurgery for intravestibular and intracochlear schwannomas. Acta Neurochir (Wien) 2019; 161: 63-67

[271] Heffner DK. Low-grade adenocarcinoma of probable endolymphatic sac origin A clinicopathologic study of 20 cases. Cancer 1989; 64: 2292-2302

[272] Lonser RR, Baggenstos M, Kim H] et al. The vestibular aqueduct: site of origin of endolymphatic sac tumors. J Neurosurg 2008; 108 : 751-756

[273] Kirsh ER, Kozin ED, Knoll RM et al. Sequential Imaging in Patient With Suspected Meniere's Disease Identifies Endolymphatic Sac Tumor. Otol Neurotol 2018; 39: e856-e859

[274] Schnack DT, Kiss K, Hansen S et al. Sporadic Endolymphatic Sac Tumor-A Very Rare Cause of Hearing Loss, Tinnitus, and Dizziness. J Int Adv Otol 2017; 13: 289-291
[275] Guo F, Zhang L, Mo L. Long experience for the diagnosis and treatment of sporadic endolymphatic sac tumor in a single center. Clin Neurol Neurosurg 2020; 197: 106078

[276] Kunzel ], Agaimy A, Hornung J et al. Sporadic endolymphatic sac tumor - a diagnostic and therapeutic challenge. Int J Clin Exp Pathol 2014; 7: 2641-2646

[277] Cmejrek RC, Megerian CA. Obstructing lesions of the endolymphatic sac and duct mimicking Ménière's disease. Ear Nose Throat J 2004; 83: 753-756

[278] Butman JA, Nduom E, Kim HJ et al. Imaging detection of endolymphatic sac tumor-associated hydrops. J Neurosurg 2013; 119 : 406-411

[279] Kitahara T, Maekawa C, Kizawa K et al. Endolymphatic sac tumor with overexpression of V2 receptor mRNA and inner ear hydrops. Acta Otolaryngol 2011; 131: 951-957

[280] Bausch B, Wellner U, Peyre M et al. Characterization of endolymphatic sac tumors and von Hippel-Lindau disease in the International Endolymphatic Sac Tumor Registry. Head Neck 2016; 38: E673-E679

[281] Lonser RR, Kim HJ, Butman JA et al. Tumors of the endolymphatic sac in von Hippel-Lindau disease. N Engl J Med 2004; 350: 2481-2486

[282] Li F, Zhang Y, Li W et al. Grading system and surgical approaches for endolymphatic sac tumors. Eur Arch Otorhinolaryngol 2020; DOI: 10.1007/s00405-020-06185-3

[283] Nelson T, Hu J, Bannykh S et al. Clinical response to pazopanib in a patient with endolymphatic sac tumor not associated with von Hippel-Lindau syndrome. CNS Oncol 2020; 9: CNS50

[284] Das S, Bakshi SS, Seepana R. Demystifying autoimmune inner ear disease. Eur Arch Otorhinolaryngol 2019; 276: 3267-3274

[285] Mijovic T, Zeitouni A, Colmegna I. Autoimmune sensorineural hearing loss: the otology-rheumatology interface. Rheumatology (Oxford) 2013; 52: 780-789

[286] Warnecke A, Jacobsen CS, Giesemann A. Embryologie, Fehlbildungen und seltene Erkrankungen der Cochlea. Laryngo-Rhino-Otol. 2021; accepted for publication

[287] Girasoli L, Cazzador D, Padoan R et al. Update on Vertigo in Autoimmune Disorders, from Diagnosis to Treatment. J Immunol Res 2018; 2018: 5072582

[288] Ciorba A, Corazzi V, Bianchini C et al. Autoimmune inner ear disease (AIED): A diagnostic challenge. Int J Immunopathol Pharmacol 2018; 32: 2058738418808680

[289] Nham B, Young AS, Garsia R et al. A treatable cause of vertigo. Pract Neurol 2020; 20: 338-342

[290] Triplett JD, Buzzard KA, Lubomski M et al. Immune-mediated conditions affecting the brain, eye and ear (BEE syndromes). J Neurol Neurosurg Psychiatry 2019; 90: 882-894

[291] Dettmer M, Hegemann I, Hegemann SC. Extraintestinal Crohn's disease mimicking autoimmune inner ear disease: a histopathological approach. Audiol Neurootol 2011; 16: 36-40

[292] Weisert JU, Veraguth D, Probst R. Bilateral deafness due to labyrinthitis in a patient with Crohn's disease. HNO 2012; 60: 132-134

[293] Pollak L. Bilateral vestibulopathy disclosing the diagnosis of celiac disease. Neurol Sci 2020; 41: 463-464

[294] Rahne T, Plontke S, Keyßer G. Vasculitis and the ear: a literature review. Curr Opin Rheumatol 2020; 32: 47-52

[295] Kohlert S, Bromwich M. Mobile tablet audiometry in fluctuating autoimmune ear disease. J Otolaryngol Head Neck Surg 2017; 46: 18

[296] Schüler O, Strupp M, Arbusow V et al. A case of possible autoimmune bilateral vestibulopathy treated with steroids. J Neurol Neurosurg Psychiatry 2003; 74: 825 
[297] Colvin IB. Audiovestibular manifestations of sarcoidosis: a review of the literature. Laryngoscope 2006; 116: 75-82

[298] Hain TC, Cherchi M, Yacovino DA. Bilateral Vestibular Weakness. Front Neurol 2018; 9: 344

[299] Strum D, Kim S, Shim T et al. An update on autoimmune inner ear disease: A systematic review of pharmacotherapy. Am J Otolaryngol 2020; 41: 102310

[300] García-Berrocal JR, Ibáñez A, Rodríguez A et al. Alternatives to systemic steroid therapy for refractory immune-mediated inner ear disease: A physiopathologic approach. Eur Arch Otorhinolaryngol 2006; 263: 977-982

[301] Salt AN, Plontke SK. Steroid Nomenclature in Inner Ear Therapy. Otol Neurotol 2020; 41: 722-726

[302] Van Wijk F, Staecker H, Keithley E et al. Local perfusion of the tumor necrosis factor alpha blocker infliximab to the inner ear improves autoimmune neurosensory hearing loss. Audiol Neurootol 2006; 11: 357-365

[303] Aschendorff A, Lohnstein P, Schipper J et al. Obliterated cochlea in Cogan's syndrome - implications for cochlear implant surgery. Laryngorhinootologie 2004; 83: 836-839

[304] van de Berg R, van Tilburg M, Kingma H. Bilateral Vestibular Hypofunction: Challenges in Establishing the Diagnosis in Adults. ORL J Otorhinolaryngol Relat Spec 2015; 77: 197-218

[305] Ward BK, Agrawal Y, Hoffman H] et al. Prevalence and impact of bilateral vestibular hypofunction: results from the 2008 US National Health Interview Survey. JAMA Otolaryngol Head Neck Surg 2013; 139: 803-810

[306] Ahmed RM, Hannigan IP, MacDougall HG et al. Gentamicin ototoxicity: a 23-year selected case series of 103 patients. Med J Aust 2012; 196: 701-704

[307] Miffon M, Guyot JP. Difficulties Faced by Patients Suffering from Total Bilateral Vestibular Loss. ORL J Otorhinolaryngol Relat Spec 2015; 77 241-247

[308] Zingler VC, Cnyrim C, Jahn K et al. Causative factors and epidemiology of bilateral vestibulopathy in 255 patients. Ann Neurol 2007; 61: 524-532

[309] Lucieer F, Vonk P, Guinand N et al. Bilateral Vestibular Hypofunction: Insights in Etiologies, Clinical Subtypes, and Diagnostics. Front Neurol 2016; 7: 26

[310] Crawford J. LIVING WITHOUT A BALANCING MECHANISM. Br ] Ophthalmol 1964; 48: 357-360

[311] Strupp M, Kim JS, Murofushi T et al. Bilateral vestibulopathy: Diagnostic criteria Consensus document of the Classification Committee of the Bárány Society. J Vestib Res 2017; 27: 177-189

[312] Black FO, Pesznecker S, Stallings V. Permanent gentamicin vestibulotoxicity. Otol Neurotol 2004; 25: 559-569

[313] Brandt T, Schautzer F, Hamilton DA et al. Vestibular loss causes hippocampal atrophy and impaired spatial memory in humans. Brain 2005; 128: 2732-2741

[314] Kremmyda O, Hüfner K, Flanagin VL et al. Beyond Dizziness: Virtual Navigation, Spatial Anxiety and Hippocampal Volume in Bilateral Vestibulopathy. Front Hum Neurosci 2016; 10: 139

[315] Popp P, Wulff M, Finke $K$ et al. Cognitive deficits in patients with a chronic vestibular failure. J Neurol 2017; 264: 554-563

[316] Dobbels B, Peetermans O, Boon B et al. Impact of Bilateral Vestibulopathy on Spatial and Nonspatial Cognition: A Systematic Review. Ear Hear 2019; 40: 757-765

[317] Guinand N, Boselie F, Guyot JP et al. Quality of life of patients with bilateral vestibulopathy. Ann Otol Rhinol Laryngol 2012; 121 : 471-477
[318] Sun DQ, Ward BK, Semenov YR et al. Bilateral Vestibular Deficiency: Quality of Life and Economic Implications. JAMA Otolaryngol Head Neck Surg 2014; 140: 527-534

[319] Tarnutzer AA, Bockisch C], Buffone E et al. Hierarchical Cluster Analysis of Semicircular Canal and Otolith Deficits in Bilateral Vestibulopathy. Front Neurol 2018; 9: 244

[320] Fujimoto C, Kinoshita M, Kamogashira T et al. Characteristics of vertigo and the affected vestibular nerve systems in idiopathic bilateral vestibulopathy. Acta Otolaryngol 2016; 136: 43-47

[321] Fujimoto C, Yagi M, Murofushi T. Recent advances in idiopathic bilateral vestibulopathy: a literature review. Orphanet J Rare Dis 2019; 14: 202

[322] Rosengren SM, Welgampola MS, Taylor RL. Vestibular-Evoked Myogenic Potentials in Bilateral Vestibulopathy. Front Neurol 2018; 9 : 252

[323] Zingler VC, Weintz E, Jahn K et al. Saccular function less affected than canal function in bilateral vestibulopathy. J Neurol 2008; 255: 1332-1336

[324] Agrawal Y, Bremova T, Kremmyda O et al. Semicircular canal, saccular and utricular function in patients with bilateral vestibulopathy: analysis based on etiology. J Neurol 2013; 260: 876-883

[325] Vital D, Hegemann SC, Straumann D et al. A new dynamic visual acuity test to assess peripheral vestibular function. Arch Otolaryngol Head Neck Surg 2010; 136: 686-691

[326] Tarnutzer AA, Bockisch C], Buffone E et al. Disease-specific sparing of the anterior semicircular canals in bilateral vestibulopathy. Clin Neurophysiol 2016; 127: 2791-2801

[327] Halmagyi GM, Curthoys IS. Adverse effects of a single dose of gentamicin. Br J Clin Pharmacol 2018; 84: 2936

[328] Dean L. "Gentamicin Therapy and MT-RNR1 Genotype". In: Pratt VM, Scott SA, Pirmohamed M et al, Hrsg. "Medical Genetics Summaries". Bethesda (MD): National Center for Biotechnology Information (US); 2012

[329] Elstner M, Schmidt C, Zingler VC et al. Mitochondrial 12S rRNA susceptibility mutations in aminoglycoside-associated and idiopathic bilateral vestibulopathy. Biochem Biophys Res Commun 2008; 377: 379-383

[330] Hirvonen TP, Minor LB, Hullar TE et al. Effects of intratympanic gentamicin on vestibular afferents and hair cells in the chinchilla. J Neurophysiol 2005; 93: 643-655

[331] Lyford-Pike S, Vogelheim C, Chu E et al. Gentamicin is primarily localized in vestibular type I hair cells after intratympanic administration. J Assoc Res Otolaryngol 2007; 8: 497-508

[332] Dobie RA, Black FO, Pezsnecker SC et al. Hearing loss in patients with vestibulotoxic reactions to gentamicin therapy. Arch Otolaryngol Head Neck Surg 2006; 132: 253-257

[333] Curthoys IS, MacDougall HG, Vidal PP et al. Sustained and Transient Vestibular Systems: A Physiological Basis for Interpreting Vestibular Function. Front Neurol 2017; 8: 117

[334] Dulon D, Hiel H, Aurousseau C et al. Pharmacokinetics of gentamicin in the sensory hair cells of the organ of Corti: rapid uptake and long term persistence. C R Acad Sci III 1993; 316: 682-687

[335] Edson RS, Brey RH, McDonald TJ et al. Vestibular toxicity due to inhaled tobramycin in a patient with renal insufficiency. Mayo Clin Proc 2004; 79: 1185-1191

[336] Ahya VN, Doyle AM, Mendez JD et al. Renal and vestibular toxicity due to inhaled tobramycin in a lung transplant recipient. J Heart Lung Transplant 2005; 24: 932-935

[337] Kaufman AC, Eliades SJ. Vestibulotoxicity in a patient without rena failure after inhaled tobramycin. Am J Otolaryngol 2019; 40: 456-458 
[338] Li W, You D, Chen Y et al. Regeneration of hair cells in the mammalian vestibular system. Front Med 2016; 10: 143-151

[339] Black FO, Gianna-Poulin C, Pesznecker SC. Recovery from vestibular ototoxicity. Otol Neurotol 2001; 22: 662-671

[340] Martin-Sanz E, Diaz JY, Esteban-Sanchez J et al. Delayed Effect and Gain Restoration After Intratympanic Gentamicin for Menière's Disease. Otol Neurotol 2019; 40: 79-87

[341] Burns JC, Stone JS. Development and regeneration of vestibular hair cells in mammals. Semin Cell Dev Biol 2017; 65: 96-105

[342] Walther LE, Hülse R, Lauer K et al. Current aspects of ototoxicity: Local ototoxic effects, diagnosis, prevention, and treatment. HNO 2015; 63: 383-392

[343] Rinne T, Bronstein AM, Rudge P et al. Bilateral loss of vestibular function: clinical findings in 53 patients. J Neurol 1998; 245: 314-321

[344] Szmulewicz D], Roberts L, McLean CA et al. Proposed diagnostic criteria for cerebellar ataxia with neuropathy and vestibular areflexia syndrome (CANVAS). Neurol Clin Pract 2016; 6: 61-68

[345] Strupp M, Hüfner K, Sandmann R et al. Central oculomotor disturbances and nystagmus: a window into the brainstem and cerebellum. Dtsch Arztebl Int 2011; 108: 197-204

[346] Gandor F, Tesch M, Neuhauser $\mathrm{H}$ et al. Diagnostic accuracy of a smartphone bedside test to assess the fixation suppression of the vestibulo-ocular reflex: when nothing else matters. J Neurol 2020; 267: 2159-2163

[347] NOVEL. "Neuro-Ophthalmology Virtual Education Library" Online: https://novel.utah.edu/ abgerufen am 24.10.2020

[348] Feil K, Böttcher N, Kremmyda O et al. Pharmacotherapy of Vestibular Disorders, Nystagmus and Cerebellar Disorders. Laryngorhinootologie 2018; 97: 14-23

[349] Lee SY, Lee DH, Bae Y] et al. Bilateral Vestibulopathy in Superficial Siderosis. Front Neurol 2018; 9: 422

[350] Weekamp HH, Huygen PL, Merx JL et al. Longitudinal analysis of hearing loss in a case of hemosiderosis of the central nervous system. Otol Neurotol 2003; 24: 738-742

[351] Kang KW, Lee C, Kim SH et al. Bilateral Vestibulopathy Documented by Video Head Impulse Tests in Superficial Siderosis. Otol Neurotol 2015; 36: 1683-1686

[352] Kumar N. Superficial siderosis: associations and therapeutic implications. Arch Neurol 2007; 64: 491-496

[353] Kumar R, Jacob JT, Welker KM et al. Superficial siderosis of the central nervous system associated with incomplete dural closure following posterior fossa surgery: report of 3 cases. J Neurosurg 2015; 123: 1326-1330

[354] Frejo L, Giegling I, Teggi R et al. Genetics of vestibular disorders: pathophysiological insights. J Neurol 2016; 263 (Suppl 1): S45-S53

[355] Frejo L, Requena T, Okawa S et al. Regulation of Fn14 Receptor and NF-kB Underlies Inflammation in Meniere's Disease. Front Immunol 2017; 8: 1739

[356] Bächinger D, Luu NN, Kempfle JS et al. Vestibular Aqueduct Morphology Correlates With Endolymphatic Sac Pathologies in Menière's Disease - A Correlative Histology and Computed Tomography Study. Otol Neurotol 2019; 40: e548-e555

[357] Bächinger D, Brühlmann C, Honegger T et al. Endotype-Phenotype Patterns in Meniere's Disease Based on Gadolinium-Enhanced MRI of the Vestibular Aqueduct. Front Neurol 2019; 10: 303

[358] Koenekoop RK, Arriaga MA, Trzupek KM et al. "Usher Syndrome Type I". In: Adam MP, Ardinger HH, Pagon RA et al, Hrsg. "GeneReviews(®)". Seattle (WA): University of Washington; 1993-2020

[359] Nolen RM, Hufnagel RB, Friedman TB et al. Atypical and ultra-rare Usher syndrome: a review. Ophthalmic Genet 2020; 41: 401-412
[360] Heuss D. “Diagnostik bei Polyneuropathien, S1-Leitlinie, 2019”. In: Deutsche Gesellschaft für Neurologie, Hrsg. "Leitlinien für Diagnostik und Therapie in der Neurologie”. Online: https://dgn.org/leitlinien/ II-030-067-diagnostik-bei-polyneuropathien-2019/abgerufen am 11.10 .2020

[361] Jahn K, Saul AK, Elstner M et al. Vestibular rehabilitation therapy and Nintendo Wii balance board training both improve postural control in bilateral vestibulopathy. J Neurol 2018; 265: 70-73

[362] Lehnen N, Kellerer S, Knorr AG et al. Head-Movement-Emphasized Rehabilitation in Bilateral Vestibulopathy. Front Neurol 2018; 9: 562

[363] Wuehr M, Decker J, Schniepp R. Noisy galvanic vestibular stimulation: an emerging treatment option for bilateral vestibulopathy. J Neurol 2017; 264: 81-86

[364] Kingma H, Felipe L, Gerards MC et al. Vibrotactile feedback improves balance and mobility in patients with severe bilateral vestibular loss. J Neurol 2019; 266: 19-26

[365] Guyot JP, Perez Fornos A. Milestones in the development of a vestibular implant. Curr Opin Neurol 2019; 32: 145-153

[366] Sluydts M, Curthoys I, Vanspauwen R et al. Electrical Vestibular Stimulation in Humans: A Narrative Review. Audiol Neurootol 2020; 25: 6-24

[367] Dlugaiczyk J, Wühr M, Straka H. "Electrical Stimulation of Vestibular Endorgans". In: Fritzsch B, Straka H, Hrsg. "The Senses: A Comprehensive Reference“. Amsterdam: Elsevier; 2020: 635-671

[368] Boutros P], Schoo DP, Rahman M et al. Continuous vestibular implant stimulation partially restores eye-stabilizing reflexes. JCI Insight. 2019; 4:

[369] Della Santina CC, Migliaccio AA, Hayden R et al. Current and future management of bilateral loss of vestibular sensation - an update on the Johns Hopkins Multichannel Vestibular Prosthesis Project. Cochlear Implants Int 2010; 11: 2-11

[370] Hageman KN, Chow MR, Roberts D et al. Binocular 3D otolith-ocular reflexes: responses of chinchillas to prosthetic electrical stimulation targeting the utricle and saccule. J Neurophysiol 2020; 123: 259-276

[371] Crane BT, Carey JP, McMenomey S et al. Meningioma causing superior canal dehiscence syndrome. Otol Neurotol 2010; 31: 1009-1010

[372] Koo JW, Hong SK, Kim DK et al. Superior semicircular canal dehiscence syndrome by the superior petrosal sinus. J Neurol Neurosurg Psychiatry 2010; 81: 465-467

[373] McCall AA, McKenna M], Merchant SN et al. Superior canal dehiscence syndrome associated with the superior petrosal sinus in pediatric and adult patients. Otol Neurotol 2011; 32: 1312-1319

[374] Liu Z, Bi W, Li J et al. Superior semicircular canal dehiscence in relation to the superior petrosal sinus: a potential cause of pulsatile tinnitus. Clin Radiol 2015; 70: 943-947

[375] Schneiders SMD, Rainsbury JW, Hensen EF et al. Superior petrosal sinus causing superior canal dehiscence syndrome. J Laryngol Otol 2017; 131: 593-597

[376] Sweeney AD, O'Connell BP, Patel NS et al. Superior Canal Dehiscence Involving the Superior Petrosal Sinus: A Novel Classification Scheme. Otol Neurotol 2018; 39: e849-e855

[377] Preet K, Udawatta M, Duong C et al. Bilateral Superior Semicircular Canal Dehiscence Associated with Ehlers-Danlos Syndrome: A Report of 2 Cases. World Neurosurg 2019; 122: 161-164

[378] Brantberg K, Bagger-Sjöbäck D, Mathiesen T et al. Posterior canal dehiscence syndrome caused by an apex cholesteatoma. Otol Neurotol 2006; 27: 531-534

[379] Fowler J, Dhaliwal S, Parnes LS. Congenital Cholesteatoma of the Mastoid Causing Posterior Semicircular Canal Dehiscence. Otol Neurotol 2019; 40: e56-e57 
[380] Russo JE, Crowson MG, DeAngelo E] et al. Posterior semicircular canal dehiscence: $\mathrm{CT}$ prevalence and clinical symptoms. Otol Neurotol 2014; 35: 310-314

[381] Whyte J, Cisneros Al, Garcia-Barrios A et al. Association between superior semicircular canal dehiscence and other dehiscences in temporal bone. Folia Morphol (Warsz) 2020; 79: 823-828

[382] Goddard JC, Oliver ER, Meyer TA. Bilateral posterior semicircular canal dehiscence in the setting of Hallermann-Streiff syndrome. Ear Nose Throat J 2012; 91: 362-363

[383] Taylor RL, Chen L, Lechner C et al. Vestibular schwannoma mimicking horizontal cupulolithiasis. J Clin Neurosci 2013; 20: 1170-1173

[384] Slattery EL, Babu SC, Chole RA et al. Intralabyrinthine schwannomas mimic cochleovestibular disease: symptoms from tumor mass effect in the labyrinth. Otol Neurotol 2015; 36: 167-171

[385] Jen JC, Wang $\mathrm{H}$, Lee $\mathrm{H}$ et al. Suggestive linkage to chromosome $6 q$ in families with bilateral vestibulopathy. Neurology 2004; 63: 2376-2379

[386] Kim BG, Kim JY, Kim HN et al. Developmental changes of ENaC expression and function in the inner ear of pendrin knock-out mice as a perspective on the development of endolymphatic hydrops. PLoS One 2014; 9: e95730

[387] Everett LA, Belyantseva IA, Noben-Trauth K et al. Targeted disruption of mouse Pds provides insight about the inner-ear defects encountered in Pendred syndrome. Hum Mol Genet 2001; 10: 153-161

[388] Nakaya K, Harbidge DG, Wangemann P et al. Lack of pendrin $\mathrm{HCO}$ - transport elevates vestibular endolymphatic $[\mathrm{Ca} 2+]$ by inhibition of acid-sensitive TRPV5 and TRPV6 channels. Am J Physiol Renal Physiol 2007; 292: F1314-F1321

[389] Dror AA, Politi Y, Shahin $\mathrm{H}$ et al. Calcium oxalate stone formation in the inner ear as a result of an Slc26a4 mutation. J Biol Chem 2010; 285: $21724-21735$

[390] Greco A, Gallo A, Fusconi M et al. Cogan's syndrome: an autoimmune inner ear disease. Autoimmun Rev 2013; 12: 396-400

[391] Mora P, Calzetti G, Ghirardini S et al. Cogan's syndrome: State of the art of systemic immunosuppressive treatment in adult and pediatric patients. Autoimmun Rev 2017; 16: 385-390

[392] D'Aguanno V, Ralli M, de Vincentiis M et al. Optimal management of Cogan's syndrome: a multidisciplinary approach. J Multidiscip Healthc 2018; 11: 1-11

[393] Kim JG, Lee SU, Lee CN et al. Bilateral vestibulopathy as an early manifestation of systemic lupus erythematosus. J Neurol 2020; 267 : 1855-1858

[394] Vos FI, Merkus P, van Nieuwkerk EB et al. Rare cause of bilateral sudden deafness. BMJ Case Rep 2016; 2016: bcr2016216004

[395] Longo L, Greco A, Rea A et al. Relapsing polychondritis: A clinical update. Autoimmun Rev 2016; 15: 539-543

[396] Strupp M, Jahn K, Brandt T. Another adverse effect of aspirin: bilateral vestibulopathy. J Neurol Neurosurg Psychiatry 2003; 74: 691

[397] Hertel S, Schwaninger M, Helmchen C. Combined toxicity of penicillin and aspirin therapy may elicit bilateral vestibulopathy. Clin Neurol Neurosurg 2013; 115: 1114-1116

[398] Gürkov R, Manzari L, Blödow A et al. Amiodarone-associated bilateral vestibulopathy. Eur Arch Otorhinolaryngol 2018; 275: 823-825
[399] Chansky PB, Werth VP. Accidental hydroxychloroquine overdose resulting in neurotoxic vestibulopathy. BMJ Case Rep 2017; 2017: bcr2016218786

[400] Fischer CS, Bayer O, Strupp M. Transient bilateral vestibular dysfunction caused by intoxication with low doses of styrene. Eur Arch Otorhinolaryngol 2014; 271: 619-623

[401] Fife TD, Robb MJA, Steenerson KK et al. Bilateral Vestibular Dysfunction Associated With Chronic Exposure to Military Jet Propellant Type-Eight Jet Fuel. Front Neurol 2018; 9: 351

[402] Weber KP, Schweier C, Kana V et al. Wear and tear vision. J Neuroophthalmol 2015; 35: 82-85

[403] Kattah JC. The Spectrum of Vestibular and Ocular Motor Abnormalities in Thiamine Deficiency. Curr Neurol Neurosci Rep 2017; 17: 40

[404] Elangovan S, Spankovich C. Diabetes and Auditory-Vestibular Pathology. Semin Hear 2019; 40: 292-299

[405] Lee SH, Kim SH, Kim JM et al. Vestibular Dysfunction in Wernicke's Encephalopathy: Predominant Impairment of the Horizontal Semicircular Canals. Front Neurol 2018; 9: 141

[406] Frohman EM, Tusa R, Mark AS et al. Vestibular dysfunction in chronic inflammatory demyelinating polyneuropathy. Ann Neurol 1996; 39: 529-535

[407] Poretti A, Palla A, Tarnutzer AA et al. Vestibular impairment in patients with Charcot-Marie-tooth disease. Neurology 2013; 80: 2099-2105

[408] Blanquet M, Petersen JA, Palla A et al. Vestibulo-cochlear function in inflammatory neuropathies. Clin Neurophysiol 2018; 129: 863-873

[409] Murofushi T, Ouvrier RA, Parker GD et al. Vestibular abnormalities in CHARGE association. Ann Otol Rhinol Laryngol 1997; 106: 129-134

[410] Satar B, Mukherji SK, Telian SA. Congenital aplasia of the semicircular canals. Otol Neurotol 2003; 24: 437-446

[411] Yukawa K, Horiguchi S, Suzuki M. Congenital inner ear malformations without sensorineural hearing loss. Auris Nasus Larynx 2008; 35: $121-126$

[412] Young AS, Taylor RL, McGarvie LA et al. Bilateral sequential peripheral vestibulopathy. Neurology 2016; 86: 1454-1456

[413] Farschtschi S, Mautner VF, McLean ACL et al. The Neurofibromatoses. Dtsch Arztebl Int 2020; 117: 354-360

[414] Jacob A, Brightman RP, Welling DB. Bilateral cerebellopontine angle metastatic melanoma: a case report. Ear Nose Throat J 2007; 86: 388-390

[415] Ito T, Sakakibara R, Komatsuzaki A et al. Aseptic meningoencephalitis presenting with bilateral vestibular ataxia: a case report. Intern Med 2006; 45: 551-552

[416] Agrawal Y, Van de Berg R, Wuyts F et al. Presbyvestibulopathy: Diagnostic criteria Consensus document of the classification committee of the Bárány Society. J Vestib Res 2019; 29: 161-170

[417] Yetişer S. Bilateral Vestibulopathy Due to Severe Cochlear Otosclerosis: A Well-Known Condition Without Any Favorable Solution. Turk Arch Otorhinolaryngol 2018; 56: 174-176

[418] MacDougall HG, McGarvie LA, Halmagyi GM et al. A new saccadic indicator of peripheral vestibular function based on the video head impulse test. Neurology 2016; 87: 410-418 